\title{
Aktualisierte S3-Leitlinie der Deutschen Gesellschaft für Gastroenterologie, Verdauungs- und Stoffwechselkrankheiten (DGVS) und der Deutschen Gesellschaft für Allgemein- und Viszeralchirurgie (DGAV) zur Prävention, Diagnostik und Behandlung von Gallensteinen
}

AWMF-Register-Nr. 021/008

\section{Updated S3-Guideline for Prophylaxis, Diagnosis and Treatment of Gallstones. German Society for Digestive and Metabolic Diseases (DGVS) and German Society for Surgery of the Alimentary Tract (DGAV)}

AWMF Registry 021/008

\begin{abstract}
Autoren
Carsten Gutt ${ }^{1}$, Christian Jenssen ${ }^{2}$, Ana-Paula Barreiros ${ }^{3}$, Thorsten O. Götze ${ }^{4}$, Caroline S. Stokes ${ }^{5}$, Petra Lynen Jansen ${ }^{6}$, Michael Neubrand ${ }^{7}$, Frank Lammert ${ }^{5,6}$, für die Teilnehmer der Konsensuskonferenz*

Weitere beteiligte Fachgesellschaften:

Deutsche Gesellschaft für Innere Medizin e.V. (DGIM), Österreichische Gesellschaft für Gastroenterologie und Hepatologie (ÖGGH), Schweizer Gesellschaft für Gastroenterologie (SGH), Gesellschaft für Humangenetik (GfH), Deutsche Gesellschaft für Ultraschall in der Medizin (DEGUM), Deutsche Gesellschaft für Chirurgie (DGCH), Institut für Qualitätssicherung und Transparenz im Gesundheitswesen (IQTIG; beratende Funktion ohne Stimmrecht), Deutsche Arbeitsgemeinschaft zum Studium der Leber (GASL), Deutsche Röntgengesellschaft (DRG), Deutsche Leberhilfe e. V.
\end{abstract}

Institute

1 Klinik f. Allgemein-, Viszeral-, Gefäß- und Thoraxchirurgie, Klinikum Memmingen, Deutschland

2 Innere Medizin, Krankenhaus Märkisch Oderland GmbH, Wriezen, Deutschland

3 Deutsche Stiftung Organtransplantation (DSO), Mainz, Deutschland

4 Institut für Klinisch-Onkologische Forschung, Krankenhaus Nordwest, Frankfurt, Deutschland

5 Klinik für Innere Medizin II - Gastroenterologie und Endokrinologie, Universitätsklinikum des Saarlandes und Medizinische Fakultät der Universität des Saarlandes, Homburg, Deutschland

6 Deutsche Gesellschaft für Gastroenterologie, Verdauungsund Stoffwechselerkrankungen, Berlin, Deutschland

7 Abteilung Innere Medizin, Krankenhaus Maria Stern, Remagen, Deutschland

Walter Asperger, Christiane Bruns, Christoph Dietrich, Klaus Dirks, Jens Encke, Siegbert Faiss, Hubertus Feussner, Thomas Frieling, Matthias Glanemann, Jochen Hampe, Hermann Helmberger, Thomas P. Hüttl, Ralf Jakobs, Birgit Kallinowski, Reinhard Kubale, Erdmute Kunstmann, Matthias Maier, Stephan Miehlke, Jürgen Pauletzki, Steffen Rickes, Andreas Schreyer, Michael Sackmann, Peter Sauer, Tilman Sauerbruch, Michael Schepke, Florian Schreiber, Jochen Schuld, Albrecht Stier, Ingo van Thiel und Marty Zdichavsky.
Schlüsselwörter

Cholithiasis, Choledocholithiasis, Cholezystitis, Cholezystolithiasis, Gallensteine

Key words

gallstones, cholelithiasis, cholecystolithiasis, choledocholithiasis, cholecystitis

eingereicht 03.05 .2018

akzeptiert 14.06.2018

Bibliografie

DOI https://doi.org/10.1055/a-0644-2972

Z Gastroenterol 2018; 56: 912-966

(c) Georg Thieme Verlag KG, Stuttgart · New York

ISSN 0044-2771

Korrespondenzadresse

Prof. Dr. Carsten Gutt

Klinik für Allgemein-, Viszeral-, Thorax- und Gefäßchirurgie Klinikum Memmingen, Bismarckstraße 23,

87700 Memmingen

Tel.: ++ 49/8331/7023 52

carsten.gutt@klinikum-memmingen.de

Prof. Dr. Frank Lammert

Klinik für Innere Medizin II (Gastroenterologie, Hepatologie, Endokrinologie, Diabetologie und Ernährungsmedizin) 
Universitätsklinikum des Saarlandes

Universität des Saarlandes, Kirrberger Straße 100,

66421 Homburg

Tel.: ++ 49/6841/16/23201

frank.lammert@uks.eu

\section{ZUSAMMENFASSUNG}

Die Leitlinie zur Prävention, Diagnostik und Therapie von Gallensteinen aktualisiert die Leitlinie der Deutschen Gesellschaft für Gastroenterologie, Verdauungs- und Stoffwechselkrankheiten und der Deutschen Gesellschaft für Allgemein- und Viszeralchirurgie aus dem Jahr 2007. Die Leitlinie wurde interdisziplinär von Gastroenterologen und Chirurgen erstellt. Sie basiert auf den Empfehlungen der Arbeitsgemeinschaft der Wissenschaftlichen Medizinischen Fachgesellschaften für eine systematische evidenzbasierte Konsensus-Leitlinie der Entwicklungsstufe S3 und berücksichtigt die klinische Anwendbarkeit der Studienergebnisse. Zudem werden Präventi- onsmöglichkeiten beschrieben, Qualitätsindikatoren definiert, ökonomische Auswirkungen berücksichtigt und Vorschläge zur sektorenübergreifenden Behandlung von Patienten mit Gallensteinerkrankungen formuliert.

\section{ABSTRACT}

This guideline provides evidence-based key recommendations for the prevention, diagnosis and therapy of gallstones and upgrades the 2007 version. The guideline was developed by an interdisciplinary team of gastroenterologists and surgeons, and patient support groups under the auspice of the German Society for Gastroenterology and Metabolic Diseases and the German Society for General Surgery and Surgery of the Alimentary Tract. The guideline used structural S3 consensusbased methodology and includes statements on clinical practice, medical education, prevention, quality assurance, outcome analysis, and integration of outpatient and inpatient care for patients with gallstone diseases.

\section{Besonderer Hinweis}

\begin{tabular}{|l|l|l|}
\hline \multicolumn{2}{|l|}{ Inhaltsverzeichnis } & Seite \\
\hline I. & Prävention & 917 \\
\hline II. & Diagnostik & 920 \\
\hline III. & Therapie & 930 \\
\hline IIIA. & Konservative Therapie & 930 \\
\hline IIIB. & Chirurgische Therapie & 931 \\
\hline IIIC. & Endoskopisch-interventionelle Therapie & 939 \\
\hline IV. & Qualitätssicherung & 945 \\
\hline Tabellen & & 948 \\
\hline Abkürzungen & 948 \\
\hline Literatur & & 949 \\
\hline
\end{tabular}

Die Medizin unterliegt einem fortwährenden Entwicklungsprozess, sodass alle Angaben, insbesondere zu diagnostischen und therapeutischen Verfahren, nur dem Wissensstand zurzeit der Drucklegung der Leitlinie entsprechen können. Hinsichtlich der angegebenen Empfehlungen zur Therapie und der Auswahl sowie Dosierung von Medikamenten wurde die größtmögliche Sorgfalt beachtet. Gleichwohl werden die Benutzer aufgefordert, die Beipackzettel und Fachinformationen der Hersteller zur Kontrolle heranzuziehen und im Zweifelsfall einen Spezialisten zu konsultieren. Fragliche Unstimmigkeiten sollen bitte im allgemeinen Interesse der DGVS mitgeteilt werden. Der Benutzer selbst bleibt verantwortlich für jede diagnostische und therapeutische Applikation, Medikation und Dosierung.

In dieser Leitlinie sind eingetragene Warenzeichen (geschützte Warennamen) nicht besonders kenntlich gemacht. Es kann also aus dem Fehlen eines entsprechenden Hinweises nicht geschlossen werden, dass es sich um einen freien Warennamen handelt.
Das Werk ist in allen seinen Teilen urheberrechtlich geschützt. Jede Verwertung außerhalb der Bestimmung des Urhebergesetzes ist ohne schriftliche Zustimmung der DGVS unzulässig und strafbar. Kein Teil des Werkes darf in irgendeiner Form ohne schriftliche Genehmigung reproduziert werden. Dies gilt insbesondere für Vervielfältigungen, Übersetzungen und Mikroverfilmungen oder die Einspeicherung, Nutzung und Verwertung in elektronischen Systemen, Intranets und dem Internet.

\section{Prävention}

\section{EMPFEHLUNG I. 1 .}

Regelmäßige körperliche Aktivität und bedarfsgerechte Ernährung zur Erhaltung eines möglichst normalen Körpergewichts können der Entwicklung von Cholesteringallenblasensteinen und biliären Symptomen vorbeugen (Statement, III, starker Konsens).

\section{Kommentar:}

Diese Leitlinienempfehlung basiert auf der S3-Leitlinie zur Prävention und Therapie der Adipositas [1]. Diese Leitlinie weist darauf hin, dass zu der Frage, welche Präventionsmaßnahmen besonders geeignet und effektiv sind, vergleichsweise wenige valide Untersuchungen existieren und dass die Studien nicht ausreichend belastbar sind, um konkrete Empfehlungen zur Art der Präventionsmaßnahmen zu formulieren. Die meisten Studien untersuchen verschiedene Maßnahmen mit unterschiedlicher Dauer [2 - 5]. Körperliche Inaktivität, kalorienreiche und ballaststoffarme Ernährung, Übergewicht und Adipositas sind etablierte exogene Risikofaktoren für die Bildung von Cholesterinsteinen in der Gallenblase [6]. Grundsätzlich scheint ein Lebensstil mit regelmäßiger körperlicher Bewegung und bedarfsadäquater Ernährung 
sinnvoll zu sein, um das Gallensteinrisiko zu senken [7 - 11]. Studienergebnisse zeigen, dass sowohl vermehrte Bewegung, Ernährungsumstellung als auch Gewichtskontrollen eine Gewichtszunahme effektiv verhindern können. Die Studien sind jedoch sehr unterschiedlich hinsichtlich des Designs, und die untersuchten Maßnahmen differieren [12-22].

In mehreren Studien wurde untersucht, wie sich der Verzehr von verschiedenen Lebensmitteln auf das Gewicht auswirkt. In systematischen Reviews des World Cancer Research Fund wurde analysiert, welche Auswirkungen verschiedene Nahrungsmittel auf das Gewicht haben [23]. Der Verzehr von energieärmeren Nahrungsmitteln (z. B. Vollkornprodukte, Getreide, Obst, Gemüse, Salat) wirkt wahrscheinlich einer Gewichtszunahme, Übergewicht und Adipositas entgegen. Der Verzehr von energiedichten Nahrungsmitteln (z. B. tierische Fette, andere Lebensmittel mit hohem Fettanteil, Süßigkeiten) verursacht dagegen wahrscheinlich eine Gewichtszunahme. Der Verzehr großer Mengen zuckerhaltiger Getränke kann eine Gewichtszunahme, Übergewicht und Adipositas begünstigen. Weitere systematische Arbeiten und Einzelstudien zeigen, dass Erwachsene eher ihr Körpergewicht stabil halten, wenn sie den Konsum von sehr energiereichen Nahrungsmitteln reduzieren und diese durch eine fettarme, ballaststoffreiche Kost mit mehr Obst, Vollkornprodukten, Gemüse und Salat ersetzen sowie weniger Süßigkeiten und energiehaltige Getränke verzehren [16, 17, 20, 21, 24, 25]. Maßnahmen zur Gewichtskontrolle sollten zudem die Energiedichte der Nahrung berücksichtigen; diese kann durch einen erhöhten Konsum wasser- und ballaststoffreicher Lebensmittel wie Gemüse und Obst und einen niedrigen Konsum von Lebensmitteln mit zugesetztem Zucker bzw. energieliefernden Getränken reduziert werden [1].

Es wurde auch berichtet, dass Obst und Gemüse bei Frauen das Risiko für eine Cholezystektomie absenken können [26, 27]. Zudem erhöht eine hohe Kohlenhydratzufuhr, insbesondere aus raffiniertem Zucker, das Risiko von Gallensteinen [28-30]. Auf der anderen Seite haben große populationsbasierte, prospektive Studien gezeigt, dass ballaststoffreiche und kalziumreiche Diäten die lithogenen Effekte hydrophober Gallensäuren reduzieren [31].

Es ist unklar, ob eine vegetarische Ernährung das Steinrisiko positiv oder negativ beeinflusst [32-37], jedoch können aus dem mit ihr assoziierten niedrigeren BMI [38] und der Verwendung von pflanzlichen Ölen protektive Effekte resultieren [30]. Eine prospektive Kohortenstudie bei französischen Frauen berichtete von einem niedrigeren Risiko einer Cholezystektomie unter einer Ernährung, die reich an Obst, Gemüse, Hülsenfrüchten und Olivenöl war [39]. Nüsse könnten auch gegen Gallensteine schützen oder einen Indikator einer gesünderen Ernährung darstellen [40]. Es konnte auch gezeigt werden, dass mehrfach und einfach ungesättigte Fette [41] protektiv sind, wohingegen eine höhere Aufnahme von gesättigten und trans-Fettsäuren mit einem erhöhten Risiko von Gallensteinen assoziiert war [42, 43].

Eine höhere Zufuhr von Vitamin C reduziert wahrscheinlich das Steinrisiko [44-47]. In einer deutschen Beobachtungsstudie mit 2129 Teilnehmern lag die sonografisch dokumentierte Prävalenz von Gallensteinen bei 4,7 \% bei den 232 Patienten, die regelmäßig Vitamin C supplementierten, im Vergleich zu 8,2\% bei den 1897 Teilnehmern, die kein Vitamin C einnahmen [48].
In einer großen prospektiven Studie wurde darüber berichtet, dass die Magnesiumaufnahme aus Nahrungsquellen bei Männern gegenüber symptomatischen Gallensteinen protektiv ist [49]. Die Studienlage in Bezug auf Kaffee ist kontrovers [35, 50 - 57].

Im Hinblick auf den Schutz gegen Gallensteinbildung während der Gewichtsabnahme haben Diäten mit höherem Fettanteil positive Wirkungen in randomisierten kontrollierten Studien gezeigt, in denen adipöse Patienten kalorienreduzierte Diäten erhielten [59-61]. Eine randomisierte Studie berichtete von keinen Unterschieden in der Gallensteinbildung bei adipösen Patienten, die während der Gewichtsabnahme zusätzlich Ballaststoff erhielten, im Vergleich zu denen, die Ursodeoxycholsäure (UDCA, siehe I.2a) erhielten [62]. Die Wirkung von $\omega$-3-Fettsäuren im Vergleich zu UDCA und Placebo wurde in einer randomisierten Studie untersucht [63], da eine Abnahme der Cholesterinsättigung der Galle bei Steinträgern berichtet wurde [64]. Keine der übergewichtigen Frauen entwickelte nach einer sechswöchigen Diät Gallensteine, jedoch wurde hier keine Abnahme des Cholesterinsättigungsindexes beobachtet. Bei Patienten mit Hypertriglyzeridämie besserte sich die Hypomotilität der Gallenblase unter einer Supplementation mit $\omega$-3-Fettsäuren [65].

Die Rolle körperlicher Aktivität wurde auch in einer randomisierten Studie untersucht. Diese Studie beobachtete eine Steigerung der Gallenblasenmotilität in der Interventionsgruppe mit moderatem Training, aber diese war nicht höher als in der Kontrollgruppe [66]. Eine Trainingsinterventionsstudie bei schwangeren Patienten fand ebenfalls keinen Unterschied zwischen den Gruppen in Hinblick auf die Bildung von Sludge oder Gallensteinen [67].

Aus Querschnittsstudien gibt es Hinweise, dass das Gallensteinrisiko um etwa ein Viertel abnehmen könnte, wenn die nächtlichen Nüchternperioden kürzer als $12 \mathrm{~h}$ sind [58].

\section{EMPFEHLUNG I.2.}

Eine generelle pharmakologische Prävention von Gallensteinen wird nicht empfohlen (Expertenkonsens).

\section{Kommentar:}

Obgleich eine generelle pharmakologische Prävention von Gallensteinen nicht empfohlen wird, gibt es schwache Evidenz für einen steinpräventiven Effekt von Statinen [68]. Daher können diese bei anderer Indikation und gleichzeitigem Vorliegen von lithogenen Risikofaktoren (z. B. Übergewicht, Bewegungsmangel, positive Familienanamnese) großzügig eingesetzt werden. Interessanterweise scheint dieser Effekt auch auf Populationsebene detektierbar zu sein: Bei Statineinnahme wird eine geringere Pankreatitisinzidenz beobachtet [69], und dieser Effekt ist am ehesten darauf zurückzuführen, dass die Bildung kleiner Steine verhindert wird [70]. Trotz experimenteller Daten gibt es bisher keinen Nachweis einer steinpräventiven Wirkung von Ezetimib beim Menschen [71, 72]. 


\section{AUSNAHMEN}

a) Bei hohem Risiko zur Bildung von Gallenblasen-Sludge oder -steinen (z. B. infolge von Gewichtsreduktion durch Reduktionsdiät oder nach Adipositaschirurgie) wird das Steinrisiko durch eine zeitlich begrenzte Prophylaxe mit Ursodeoxycholsäure vermindert (Statement, I, starker Konsens).

\section{Kommentar:}

Adipositas per se und insbesondere abdominelle Adipositas prädisponieren zur Gallensteinbildung und erhöhen das Risiko für eine Cholezystektomie wegen symptomatischer Gallensteine [73 - 75], während körperliche Aktivität steinprotektiv ist [7, 10, $30,44,76]$. Bei rascher Gewichtsreduktion (>1,5 kg/Woche) unter Reduktionsdiät steigt das Steinrisiko signifikant an [77-80]; Gewichtsschwankungen („Weight cycling“) sind ein unabhängiger Risikofaktor [44, 81, 82]. Gallensteine oder Sludge entstehen bei etwa $30 \%$ der Patienten innerhalb von sechs Monaten nach Magen-Bypass oder biliopankreatischer Diversion [83, 84], nicht jedoch nach laparoskopischem Magenband [85].

Randomisierte kontrollierte Studien [77, 84, 86 - 90] konnten zeigen, dass das Steinrisiko unter Reduktionsdiät mit rascher Gewichtsabnahme oder nach Magen-Bypass durch die Einnahme von Ursodeoxycholsäure (UDCA) signifikant um $58 \%$ verringert wird [61].

UDCA soll mit einer Dosis von mindestens $500 \mathrm{mg} / \mathrm{Tag}$ über mindestens 4 Monate bis zur Gewichtsstabilisierung eingenommen werden. Im Gegensatz zur medikamentösen Steinauflösung wurden in den Studien keine gewichtsbezogenen, sondern fixe UDCA-Dosen gewählt. Die Metaanalyse zeigte, dass eine UDCADosis von mindestens $500 \mathrm{mg} /$ Tag effektiver ist als $300 \mathrm{mg}$, wobei steinprotektive Effekte nach 3-4 Monaten, aber auch bereits nach 6 Wochen dokumentiert wurden. Sugerman et al. [84] dokumentierten nach sechsmonatiger UDCA-Therapie bei Patienten mit Magen-Bypass eine stärkere präventive Wirkung bei einer Dosis von $600 \mathrm{mg}$ (Steininzidenz 2\%) und $1200 \mathrm{mg}$ (6\%) im Vergleich zu $300 \mathrm{mg} / \mathrm{Tag}$ (13\%). Eine vergleichbare Wirksamkeit konnten Shiffman et al. [88] unter forcierter Diät für eine UDCADosis von 600 - 1200 mg UDCA/Tag im Vergleich mit 300 mg/Tag nachweisen (2-3 vs. $8 \%$ ). In den Studien, die den Metaanalysen zugrunde liegen, wurde 4-6 Monate behandelt. Da eine postbariatrische Cholezystektomie in der Regel problemlos durchzuführen ist, wird häufig postoperativ keine prophylaktische Gabe von UDCA begonnen [91, 92].

Hinweis zu Interessenkonflikten: Trotz der Klasse la-Evidenz (Cochrane-Review) wurde für die Steinprophylaxe mit Ursodeoxycholsäure (UDCA) keine Empfehlung formuliert, sondern nur das Statement zur Evidenz aufgenommen, da (1) das Medikament für diese Indikation keine Zulassung besitzt und (2) bei einigen Teilnehmern der Konsensuskonferenz potenzielle Interessenkonflikte mit Bezug auf einen Hersteller von UDCA bestehen (Honorare für inhaltlich nicht vorgegebene oder abgestimmte Fortbildungsvorträge, siehe Anlage I des Leitlinienreports).

\section{AUSNAHMEN}

b) Bei Auftreten von intrahepatischen Steinen oder rezidivierenden Gallengangsteinen bei jungen Patienten kann eine genetische Analyse des $A B C B 4$-Gens veranlasst werden; unabhängig davon sollte auch bei klinischer Diagnose eines Low phospholipid-associated cholelithiasis (LPAC)-Syndroms eine Therapie mit Ursodeoxycholsäure begonnen werden (Expertenkonsens).

\section{Kommentar:}

Die wichtigsten diagnostischen Kriterien des durch Mutationen im Gen des hepatokanalikulären Phospholipidtransporter ABCB4 bedingten seltenen LPAC-Syndroms sind (1) symptomatische Cholelithiasis vor dem 40. Lebensjahr, (2) sonografischer Verdacht auf intrahepatische Mikrolithiasis bzw. Sludge und (3) rezidivierende biliäre Schmerzen nach Cholezystektomie infolge von intrahepatischen oder Gallengangsteinen [93-96]. Die genetische Untersuchung erfordert die Sequenzierung des Gens, da keine häufigen „Hot-spot“-Mutationen vorliegen.

Die Therapie des LPAC-Syndroms mit UDCA sollte bereits nach klinischer Diagnosestellung begonnen werden $(10-15 \mathrm{mg} / \mathrm{kg}$ Körpergewicht und Tag), da sie unabhängig vom Mutationsnachweis ist. Aufgrund der Seltenheit der Erkrankung sind hierzu aber keine kontrollierten Daten vorhanden.

Hinweis zu Interessenkonflikten: Es wird darauf hingewiesen, dass Mitglieder der Konsensuskonferenz potenzielle Interessenkonflikte mit Bezug auf einen Hersteller von UDCA angegeben haben (siehe Hinweis zu Empfehlung I.2a), der Empfehlung I.2b jedoch alle anderen Mitglieder der Konsensuskonferenz einstimmig zugestimmt haben.

\section{EMPFEHLUNG I. 3.}

Bei der Nutzen-Risiko-Abwägung einer östrogenbasierten Hormontherapie soll das erhöhte Risiko für Gallenblasensteine und biliäre Symptome berücksichtigt werden (A, I, starker Konsens).

\section{Kommentar:}

Diese Empfehlung wurde von der S3-Leitlinie zur Hormontherapie in der Peri- und Postmenopause adaptiert [97]. CochraneReviews und Metaanalysen kommen zu der Schlussfolgerung, dass eine Hormontherapie das Risiko für Gallenwegserkrankungen erhöhen kann [98]. Aus pathophysiologischer Sicht ist die Lithogenität jeglicher Östrogen-Exposition der Gallenblase bekannt [99].

Die Nurses' Health Study, eine qualitativ gute Kohortenstudie, ergab ein erhöhtes Cholezystitis-Risiko unter Hormonanwendung [100]. Weitere Kohortenstudien [101, 102] und Querschnittsuntersuchungen [103, 104] kommen zu analogen Ergebnissen bezüglich erhöhter Risiken für Erkrankungen der Gallenblase und Gallenwege. In der HERS-Studie lag nach kombinierter Einnahme von konjugiertem Östrogen und Gestagen ein erhöhtes Risiko für 
Cholezystektomien im Vergleich zur Placebo-Gruppe vor [105]. Das Risiko für Gallenblasenoperationen war um 38 \% erhöht, insbesondere bei Patientinnen mit Übergewicht, Fibrattherapie oder vorausgegangenen Gallenwegserkrankungen ohne OP. Statingebrauch verringerte das Risiko. Die multizentrische, doppelt verblindete prospektiv randomisierte WHI-Studie ergab sowohl für eine Östrogen- als auch für eine kombinierte Östrogen-Progesteron-Therapie postmenopausaler Frauen eine erhöhte Ereigniswahrscheinlichkeit für jegliche biliäre Erkrankungen, Cholezystitis, Cholelithiasis und Cholezystektomien im Vergleich zur Placebo-Einnahme. Unter alleiniger Östrogentherapie kam es zu einer annähernden Verdoppelung des Cholezystektomie-Risikos [106, 107].

EPIC-Norfolk, eine prospektive Kohortenstudie hoher Qualität, ergab ein erhöhtes Risiko von symptomatischen Gallensteinen und Komplikationen unter Hormontherapie, abhängig von der Dauer der Anwendung. Symptomatische Gallensteine traten bei einer von 107 - 320 Frauen pro Jahr Hormontherapie-Anwendung auf [108]. Eine Auswertung der Million Women Study [109] zeigte, dass sich bei Hormontherapie-Anwenderinnen das Risiko einer stationären Aufnahme wegen einer Gallenblasenerkrankung erhöht. Dabei ist das Risiko unter transdermaler Therapie geringer als unter oraler Therapie. Die Risiken konjugierter Östrogene sind geringfügig höher als die von Östradiol, außerdem besteht eine Dosis-Wirkungsbeziehung, die allerdings relativ flach verläuft. Nach Therapie-Ende sank das Risiko, blieb aber noch nach zehn Jahren erhöht.

Die begleitende Gestagen-Therapie oder Tibolon-Anwendung änderten das Risiko für stationäre Aufnahmen durch Gallenblasenerkrankungen nicht wesentlich. Für Cholezystektomien waren die relativen Risiken annähernd gleich hoch wie für die oben genannten stationären Aufnahmen. Die Evidenz zur Steigerung des Risikos für Gallenwegserkrankungen bezieht sich somit insbesondere auf Effekte unter oraler Therapie mit konjugierten Östrogenen (mit und ohne Gestagen-Zusatz) [97].

EMPFEHLUNG I.4.

Zur medikamentösen Prävention von Gallengangsteinen kann keine Empfehlung ausgesprochen werden (Expertenkonsens).

\section{Kommentar:}

Die Entstehung von Rezidivsteinen im Gallengang wird durch eine bakterielle Besiedelung der Gallengänge begünstigt. Sie werden bei 5-20\% der Patienten nach EPT beobachtet und können im Regelfall erneut endoskopisch entfernt werden [110-116]. Genetische Risikofaktoren für Gallensteine wie Mutationen des hepatokanalikulären Phospholipidtransporters ABCB4 [95, 96] oder des Cholesterintransporters ABCG5 / G8 begünstigen die Entstehung von Rezidivsteinen in den Gallengängen [117]. Eine gesicherte prophylaktische Therapie besteht derzeit nicht. UDCA zeigte in einer Studie bei einer kleinen Patientengruppe und kurzem Follow-up eine leichte Reduktion der Rezidivrate. Aufgrund pathophysiologischer Überlegungen könnte auch die Kombination von UDCA und Statin sinnvoll sein, jedoch fehlen hierfür größere Fallserien [118]. Insgesamt ist die Evidenz zu dieser Fragestellung unzureichend, sodass keine Empfehlung möglich ist.

\section{EMPFEHLUNG I.5.}

Zur medikamentösen Prävention der Okklusion von Gallengangstents kann keine Empfehlung ausgesprochen werden (Expertenkonsens).

\section{Kommentar:}

Die Metaanalyse zu dieser Fragestellung [119] basierte auf drei Studien, die keine Evidenz für die Effektivität von UDCA und/oder Antibiotika zur Prävention einer Stentokklusion ergaben. Zwei Folgestudien konnten ebenfalls nicht eindeutig die Wirksamkeit dieser Maßnahmen nachweisen: Während eine Studie unter der Kombination von UDCA mit Levofloxacin eine niedrige Rate an Stentokklusionen und Cholangitiden fand [120], wurde in einer doppelblinden randomisierten Studie mit Ciprofloxacin bei $94 \mathrm{~Pa}$ tienten eine grenzwertig signifikante Abnahme der Cholangitishäufigkeit von 42 auf $21 \%$ beobachtet [121]. Retrospektive infektiologische Untersuchungen weisen darauf hin, dass Patienten mit biliären Stents, die eine akute Cholangitis entwickeln, eine empirische Antibiotikatherapie, die Enterokokken und Extended spectrum $\beta$-Laktamasen (ESBL) -bildende Enterobakterien abdeckt, erhalten sollten [122].

\section{Diagnostik}

EMPFEHLUNG II. 1.

Charakteristische Symptome für Gallenblasensteine sind gut erinnerliche Schmerzattacken von mehr als 15 min Dauer im Epigastrium oder rechten Oberbauch, die auch in den Rücken und in die rechte Schulter ausstrahlen können, und nicht selten besteht zudem Übelkeit, gelegentlich auch Erbrechen; abgesehen davon können bei den ersten Symptomen bereits Komplikationen eines Gallenblasensteinleidens vorliegen (Statement, III, starker Konsens, NKLM).

\section{Kommentar:}

Obwohl die Spezifität biliärer Symptome umstritten ist, gibt es derzeit keine bessere Definition [123]. Eine Multicenterstudie aus Italien bestätigt das signifikant häufigere Vorkommen der o. a. Beschwerden bei Gallensteinträgern [124]. Biliäre Symptome werden im Allgemeinen als „kolikartig“ beschrieben. In einer bevölkerungsbasierten dänischen Studie wurden als häufigste Symptome von Gallensteinen mehrere Stunden anhaltende, moderate bis starke Schmerzen im Epigastrium, die die Einnahme von Analgetika erforderten, beobachtet [125]. Dies unterstreicht, dass es bei Symptomen eines Gallensteinleidens keine eindeutig typische Lokalisation gibt. Für die akute Diagnostik bedeutsam ist, dass die Symptome (insbesondere bei Komplikationen) überwiegend am späten Abend und in der Nacht beginnen [125, 126]. In einer 
prospektiven Studie in Norwegen [126] waren die biliären Koliken bei der Mehrzahl der Steinpatienten mit funktionellen abdominiellen Beschwerden assoziiert. Als Begleitsymptome fanden sich in $66 \%$ Zeichen einer Nahrungsmittelintoleranz, aber nur in $48 \%$ eine Fett-Unverträglichkeit. Dyspepsie und Blähungen sind jedoch keine steintypischen Beschwerden, da sie mindestens gleich häufig bei steinfreien oder bei Patienten mit Reizdarmsyndrom auftreten [127 - 129] und nach Cholezystektomie häufig persistieren [130]. Nach erstmaliger Symptomatik entwickelt mehr als die Hälfte der Patienten im weiteren Verlauf neuerliche Schmerzattacken [131 - 134]. Die Wahrscheinlichkeit, biliäre Symptome zu entwickeln, beträgt 1 - 4\% pro Jahr [132, 134 - 138], und Komplikationen treten bei 0, 1-0,3\% der Gallensteinträger auf [132, 134 -136]. Multiple Steine gehen mit einem erhöhten Risiko einher, eine akute Cholezystitis oder symptomatische Gallengangsteine als Komplikationen der Cholelithiasis zu entwickeln [139]. Dem Patienten mit symptomatischer Cholezystolithiasis wird daher nicht nur zur Verhinderung neuerlicher Schmerzattacken, sondern auch zur Verhinderung von Komplikationen zur Cholezystektomie geraten (siehe IIIB.1).

\section{EMPFEHLUNG II.2.}

Der Nachweis oder Ausschluss einer Cholezystolithiasis soll durch eine systematisch durchgeführte transkutane Sonografie erfolgen (A, II, starker Konsens, NKLM).

\section{Kommentar:}

Die Sonografie ist die Methode der Wahl für die Diagnostik von Gallenblasensteinen mit einer Sensitivität > $95 \%$ [140, 141] und einer Spezifität von nahezu $100 \%$. Die Untersuchung sollte systematisch durchgeführt und dokumentiert werden. Dies erfordert den Einsatz der aktuellen Gerätetechnik durch erfahrene ärztliche Untersucher. Unabdingbar ist die komplette Darstellung der Gallenblase in variablen Schnittebenen und in zumindest zwei Patienten-Lagerungsvarianten, die um $90^{\circ}$ zueinander versetzt sind, um das typische gravitationsabhängige Migrationsverhalten auch kleinerer Steine zu erfassen. Beurteilt werden sollen neben dem Gallenblaseninhalt ein durch den Schallkopf über der Gallenblase auslösbarer umschriebener Druckschmerz, die Größe der Gallenblase, ihre Wandung sowie die intra- und extrahepatischen Gallenwege, um neben einer Cholezystolithiasis auch bereits bestehende Komplikationen sowie gleichzeitig bestehende Gallenblasenpolypen oder für ein Gallenblasenkarzinom typische Wandveränderungen detektieren zu können.

Die Gallenblasenübersichtsaufnahme sowie die orale oder intravenöse Cholangiografie werden zur Diagnostik von Gallensteinen nicht mehr angewandt. CT, MRT oder Endosonografie sind zum Nachweis von Gallenblasensteinen nur in seltenen Ausnahmen erforderlich. Kleine prospektive Studien haben das Potenzial der Endosonografie aufgezeigt, bei Patienten mit typischer biliärer Symptomatik und negativer Abdomensonografie in einem hohen Prozentsatz eine Mikrolithiasis der Gallenblase nachzuweisen [142, 143].

\section{EMPFEHLUNG II.3.}

Bei außergewöhnlichen klinischen Konstellationen der Cholelithiasis (wie familiärer Häufung, Auftreten im Kindes- und Jugendalter, intrahepatischen Steinen, rezidivierender Choledocholithiasis) sollten weitere Untersuchungen zur Abklärung einer sekundären Form der Cholelithiasis erfolgen. Hierbei sind insbesondere hämolytische Anämien, Gallensäureverlustsyndrome, parasitäre und bakterielle Infektionen sowie verschiedene genetisch bedingte Erkrankungen wie das Low-phospholipid associated cholelithiasis (LPAC)-Syndrom (ABCB4-Defizienz), Caroli-Syndrom, Gilbert-MeulengrachtSyndrom, Mukoviszidose und myotone Dystrophien zu berücksichtigen (Expertenkonsens, NKLM).

\section{Kommentar:}

In diesen Situationen sind anamnestische Angaben (z. B. Muskelschwäche, Muskelschmerzen, Katarakt, rezidivierende bronchopulmonale Infekte oder Gedeihstörung), die Familienanamnese und weitere pathologische Laborparameter richtungweisend. Eine erweiterte Labordiagnostik kann Aufschlüsse zur Ätiologie der Gallensteine liefern. Die Bildung „schwarzer“ Pigmentsteine (Calciumbilirubinatsteine) in der Gallenblase kann auf eine zugrundeliegende hämolytische Anämie oder eine Mukoviszidose hinweisen. Bei hämolytischer Anämie sollte die ethnische Herkunft des Patienten erfragt werden. Eine Hämoglobinelektrophorese (Sichelzellanämie, Thalassämie) und eine Testung der osmotischen Resistenz der Erythrozyten sowie ein Blutausstrich (Sphärozytose, Elliptozytose) führen dann diagnostisch weiter; zusätzlich sollte an den Glukose-6-phosphatdehydrogenase- und den Pyruvatkinase-Mangel gedacht werden. Auf die Mukoviszidose, bei der die Gallensteinprävalenz auf 15 - 30 \% im Vergleich zu 5 \% bei altersentsprechenden Kontrollen erhöht ist [144], können Diarrhöen und ein Gallensäureverlustsyndrom hinweisen.

Bei symptomatischer Cholelithiasis vor dem 40. Lebensjahr, Auftreten von biliären Schmerzen nach Cholezystektomie oder sonografischem Verdacht auf intrahepatische Mikrolithiasis bzw. Sludge kann eine mit einer ABCB4-Defizienz assoziierte Cholelithiasis vorliegen. Eine positive Familienanamnese kann den Verdacht erhärten, und es besteht ferner eine Assoziation zur intrahepatischen Schwangerschafts-Cholestase und zu einer geringen anderweitig nicht erklärten biochemischen Cholestasekonstellation [93, 94, 145]. Bei ABCB4-Defizienz ist die Phospholipidkonzentration im Verhältnis zu den Gallensäuren- und den Cholesterinkonzentrationen in der Galle vermindert [93]. Die Verdachtsdiagnose kann klinisch gestellt werden. Diagnostisch können eine Lipidanalyse von Leber- oder Duodenalgalle und eine genetische Untersuchung des hepatobiliären Phospholipidtransporters $A B C B 4$ durchgeführt werden. Bei $25 \%$ junger Patientinnen < 30 Jahre mit Gallensteinen, die nicht das typische lithogene Risikoprofil aufweisen, wurde in einer kleineren Fallserie ein LPAC-Syndrom diagnostiziert [146]. Seltene Mutationen im $A B C B 11-G e n$ des Gallensäuretransporters können infolge niedriger Gallensäurekonzentrationen ebenfalls zur Cholesterinsteinbildung führen [147]. Es gibt Hinweise darauf, dass ein Phosholipid- 
mangel auch beim Caroli-Syndrom eine ursächliche Rolle spielen kann, das auch in Assoziation mit der autosomal-rezessiven polyzystischen Nierenerkrankung (ARPKD) auftreten kann.

Generell beträgt die erbliche Komponente bei der Entstehung von Gallenblasensteinen etwa 25 \% [148 - 151]. Genetische Studien konnten nachweisen, dass eine häufige Variante des ABCG8Gens des hepatobiliären Cholesterintransporters (p.D19H) das Risiko, Cholesteringallensteine zu entwickeln, mehr als verdoppelt $[152,153]$. Eine genetische Diagnostik dieses Gens ist bei Gallensteinträgern nicht erforderlich.

Bei der myotonen Dystrophie Typ 1, für die eine Repeatexpansion am DMPK-Genlocus ursächlich ist, ist die Familienanamnese zur Erfassung möglicher Symptome (wie Katarakt, Muskelschwäche oder Tagesmüdigkeit) in den verschiedenen Organsystemen bei den Familienmitgliedern von großer Bedeutung, wobei ältere Familienmitglieder nur monosymptomatische Verläufe zeigen können.

Bei Diagnose oder Verdacht einer Cholelithiasis im Rahmen einer genetisch bedingten Erkrankung soll dem Patienten eine genetische Beratung angeboten werden (Gendiagnostikgesetz GenDG §10, Abs. 1). Ist die ursächliche genetische Veränderung bekannt, können weitere Familienmitglieder genetisch untersucht werden. Hierzu ist nach GenDG $\S 10$, Abs. 2 eine genetische Beratung im Vorfeld erforderlich.

\section{EMPFEHLUNG II.4}

Der Nachweis oder Ausschluss einer akuten Cholezystitis sowie möglicher Komplikationen soll primär sonografisch in Kombination mit den klinischen Befunden erfolgen: Charakteristisch ist die Trias von lokalen Entzündungszeichen (Murphy-Zeichen, lokale Abwehrspannung), systemischen Entzündungszeichen (Fieber, Leukozytose und CRP-Erhöhung) und Wandverdickung der Gallenblase (A, I, starker Konsens, NKLM).

\section{Kommentar:}

Die Sonografie zeigt in einer Metaanalyse zahlreicher Studien ab 1978 eine Sensitivität von 81 \% und eine Spezifität von $83 \%$ für die Diagnose der akuten Cholezystitis [154]. Gleichzeitig ermöglicht sie einen zuverlässigen Steinnachweis und die Beurteilung der intra- und extrahepatischen Gallengänge [155 - 157]. Das sonografische Murphy-Zeichen (umschriebener Schmerz unter direktem Druck mit dem Schallkopf) soll mitüberprüft werden und kann die Treffsicherheit der Methode deutlich verbessern [158].

Weitere Zeichen der akuten Cholezystitis können umgebende Flüssigkeit, Gallenblasen-Hydrops, umgebende Fettgewebsreaktion sowie eine vermehrte Wanddurchblutung sein. Wandverdickungen über 5 mm und ein umgebendes Exsudat können Hinweis für ein erhöhtes Konversions- und Komplikationsrisiko sein [159]. Bei der Differenzierung zur chronischen Cholezystitis ist ein vermehrtes Kontrastmittel-Enhancement (CEUS, KM-CT, KM-MRT) in der Gallenblasenwand oder Umgebung Hinweis für eine akute Entzündung [160 - 162]. Es kann bei schweren Erkrankungen, z. B. einer gangränösen Cholezystitis aber auch fehlen [161, 162].
CT und MRT erreichen ähnliche Sensitivität und Spezifität wie die Sonografie, sind aber u. a. aus Gründen des Strahlenschutzes (CT) und der sofortigen Verfügbarkeit (MRT) in der Regel nicht Methode der ersten Wahl [160, 163-165]. Bei unklaren Ultraschallbefunden oder Verdacht auf Komplikationen können CT und MRT aber eine sinnvolle Ergänzung sein. Die hepatobiliäre Funktionsszintigrafie ist in Deutschland zur Diagnostik der akuten Cholezystitis kein akzeptierter Standard [166].

Neuere Untersuchungen zeigen, dass eine frühe Cholezystektomie zumindest für Subgruppen vorteilhaft ist [167]. Unter diesem Aspekt ist die bildgebende Diagnostik der akuten Cholezystitis zeitnah verfügbar zu halten. Sonografische Untersuchungen bei Notfallpatienten haben bestätigt, dass der Nachweis von Gallensteinen wie auch einer Cholezystitis nicht nur durch Radiologen, sondern auch durch trainierte Fachärzte mit großer Treffsicherheit möglich ist [168-170].

\section{EMPFEHLUNG II.5.}

Diffuse Wandverdickungen der Gallenblase können sowohl bei Cholezystitis als auch bei zahlreichen anderen Erkrankungen auftreten. Eine weitere Abklärung sollte im klinischen Kontext betrachtet werden und kann die Indikation zur Operation wie auch das operative Vorgehen beeinflussen (Expertenkonsens).

\section{Kommentar:}

Neben der primären, meist Konkrement-bedingten Cholezystitis finden sich entzündliche Wandverdickungen der Gallenblase auch als Mitreaktion bei Virus-Hepatitis, viralen Erkrankungen mit Leberbeteiligung und anderen intraabdominellen Infektionen [171 - 173]. Zum Teil korreliert das Ausmaß der Wandverdickung mit der Schwere der Grunderkrankung $[174,175]$. Es besteht in der Regel keine Indikation zur Cholezystektomie, sodass die Differenzierung von der akuten Cholezystitis bedeutsam ist.

Andererseits können auch Flüssigkeitseinlagerungen oft zu einer ödematösen Wandverdickung beträchtlichen Ausmaßes führen (Leberzirrhose, Herzinsuffizienz, Niereninsuffizienz, Eiweißmangel, Schock).

Bei den hyperplastischen Gallenblasen-Erkrankungen ist die Adenomyomatose mit 2-5\% nicht selten [176]. Sie kann lokalisiert oder diffus auftreten und muss von neoplastischen Veränderungen abgegrenzt werden, insbesondere vom ebenfalls polypös oder diffus wachsenden Gallenblasenkarzinom. Die Adenomyomatose wird heute nicht mehr als präkanzeröse Läsion angesehen, sie ist aber gelegentlich schwierig von neoplastischen Wandverdickungen zu differenzieren. Neuere Studien zeigen hier eine Überlegenheit der hochauflösenden Sonografie und der kontrastverstärkten Kernspintomografie mit Magnetresonanz-cholangiopankreatografie (MRCP) $[177,178]$. Auch ein CT oder die kontrastverstärkte Endosonografie können hilfreich sein [179, 180]. Die früher zur Diagnose der Adenomyomatose verwendete orale Cholezystografie findet keine Verwendung mehr. 


\section{EMPFEHLUNG II.6.}

Vor einer Cholezystektomie sollten eine transkutane Sonografie einschließlich gezielter Beurteilung der Gallengänge durchgeführt und folgende Laborparameter bestimmt werden: Cholestase-Parameter ( $\mathrm{Y}-\mathrm{GT}, \mathrm{AP})$, ALT, Bilirubin, Lipase, globale Gerinnungstests und kleines Blutbild (B, III, starker Konsens, NKLM).

Vor einer elektiven Cholezystektomie bei unkompliziertem Gallensteinleiden sollten differenzialdiagnostisch andere Ursachen der Beschwerden, z. B. Ulcusleiden, erwogen werden (B, III, Konsens, NKLM).

\section{Kommentar:}

Die präoperative Sonografie dient dem Nachweis von Gallenblasensteinen mit und ohne Komplikationen. Die präoperative Bestimmung von Cholestaseenzymen, Transaminasen und Bilirubin ist notwendig, um den Verdacht auf Gallengangsteine oder eine vorbestehende Leberkrankheit weitgehend auszuschließen bzw. zu bestätigen [181, 182]. Im Gegensatz zur Sonografie und den Laboruntersuchungen ist eine routinemäßige Ösophagogastroduodenoskopie vor der elektiven Cholezystektomie nicht indiziert $[183,184]$. Diese wird in individuellen Fällen durchgeführt werden, wenn eine Ulcusanamnese besteht, der Patient nichtsteroidale Antirheumatika (NSAR) eingenommen hat oder die klinischen Symptome nicht typisch für eine biliäre Kolik sind.

\section{EMPFEHLUNG II.7.}

Bei klinisch-anamnestischen Hinweisen auf das Vorliegen einer Choledocholithiasis sollen Gesamtbilirubin, $\mathrm{Y}$-GT, AP, ALT (oder AST) und Lipase bestimmt und eine systematisch durchgeführte transabdominelle Sonografie durchgeführt werden (A, II, starker Konsens, NKLM).

\section{Kommentar:}

In Metaanalysen erwiesen sich vor allem das klinische Bild der Cholangitis, der transkutan-sonografische Nachweis von Gallengangkonkrementen, Ikterus, Hyperbilirubinämie und in der Sonografie dilatierte Gallengänge als starke Hinweise auf eine Choledocholithiasis [181, 185]. Weitere Studien zeigten darüber hinaus auch den prädiktiven Stellenwert einer Erhöhung der Transaminasen und der Cholestaseenzyme ( $\gamma$-GT, AP) [186 - 206]. Die Erhöhung von mindestens einem von fünf biochemischen Markern (Bilirubin, AP, Y-GT, ALT, AST) hatte in einer retrospektiven Studie eine Sensitivität von 88 \% bei einer Spezifität von lediglich 53 \% für das Vorliegen einer Choledocholithiasis [201]. In einer durch MRCP kontrollierten retrospektiven Studie erwiesen sich neben einer initialen Erhöhung der ALT oder einem pathologischen Ultraschallbefund ein Anstieg von ALT und AP bzw. die persistierende Erhöhung dieser Parameter als hoch prädiktiv für das Vorliegen einer Choledocholithiasis [206].

Die Sensitivität des transkutanen Ultraschalls für den Nachweis einer Choledocholithiasis wird in verschiedenen Studien sehr variabel zwischen 32 und $100 \%$ angegeben. Die Spezifität ist dagegen so hoch, dass der sonografische Nachweis eines Gallengangsteins als das Kriterium mit dem höchsten Voraussagewert für eine Choledocholithiasis gilt. Studien berichten über eine Sensitivität von 82 und $86 \%$ bei einer Spezifität von 88 bzw. $97 \%$ [207, 208]. Eine Cochrane-Analyse aus dem Jahr 2015 ermittelte für die sonografische Diagnose einer Choledocholithiasis eine gepoolte Sensitivität und Spezifität von 73 bzw. 91 \% [209]. Bei der Sonografie sollte in einem systematischen Untersuchungsgang sowohl nach direkten als auch nach indirekten Kriterien für Gallengangsteine gesucht werden. Im klinischen Alltag gelingt oft keine komplette Gangdarstellung bis zur Papille. Limitationen für die sonografische Detektion der Choledocholithiasis ergeben sich insbesondere bei normaler Gangweite [208], unmittelbar präpapillärer Lage von Konkrementen und einer Konkrementgröße $<5 \mathrm{~mm}$. In einer prospektiven Studie konnte gezeigt werden, dass erfahrene Untersucher eine hohe diagnostische Genauigkeit in der sonografischen Detektion von Gallengangsteinen haben ( $83 \%$; Sensitivität $82 \%$, Spezifität $88 \%$ ) und weniger erfahrenen Untersuchern (Genauigkeit $64 \%$; Sensitivität $46 \%$, Spezifität $91 \%$ ) signifikant überlegen sind [207].

Eine Gallengangerweiterung ist ein wichtiges indirektes sonografisches Kriterium für das Vorliegen einer Choledocholithiasis und hatte in der genannten Metaanalyse eine gepoolte Sensitivität von $42 \%$ und eine gepoolte Spezifität von $96 \%$ [181]. Mehrere aktuelle prospektive und retrospektive Studien bestätigen den diagnostischen Stellenwert einer Erweiterung der extra- und intrahepatischen Gallengänge für das Vorliegen von Gallengangsteinen [196, 200, 202 - 204, 206, 210]. Dabei wird in den meisten aktuellen Studien zur Diagnostik der simultanen Choledocholithiasis und in Leitlinien eine Gallengangerweiterung als Überschreitung eines Durchmessers des Ductus hepatocholedochus von 6 oder 7 mm definiert [201, 203, 204, 211, 212]. Im höheren Lebensalter nimmt der Durchmesser der nicht obstruierten extrahepatischen Gallengänge geringfügig zu, ohne jedoch bei der überwiegenden Mehrzahl der Patienten die genannten oberen Grenzwerte von 6 oder 7 mm zu überschreiten [213 - 215]. Eine fehlende Gallengangerweiterung schließt das Vorliegen einer Choledocholithiasis alleine nicht mit klinisch ausreichender Sicherheit aus [196, 206, 210, 216].

Auch Anzahl und vor allem Größe von Gallenblasensteinen haben prädiktive Bedeutung für das Vorliegen einer Choledocholithiasis [200, 217, 218]. In der prospektiven Studie von Costi et al. [217] wurde gezeigt, dass multiple kleine Gallenblasenkonkremente (Durchmesser $\leq 5 \mathrm{~mm}$ ) viermal häufiger mit Gallengangsteinen einhergehen als einzelne oder mehrere größere Konkremente.

Wenn keiner der aufgeführten klinischen, biochemischen oder sonografischen Indikatoren für eine Choledocholithiasis vorliegt, hat dies den gleichen negativen prädiktiven Wert wie eine ERC ohne Steinnachweis [200, 201, 204, 219, 220].

Bei erhöhter Serumlipase ist bei entsprechender Symptomatik an eine biliäre Pankreatitis zu denken (siehe II.11).

Mathematische Vorhersagemodelle für Gallengangsteine, die auf den genannten Parametern basieren, können eine hohe Genauigkeit erreichen, wurden aber nur in wenigen Fällen in einer unabhängigen Kohorte prospektiv validiert und sind in der klini- 
schen Praxis kaum anwendbar [182, 200, 205, 221]. Durch Nutzung klinischer Variablen, laborchemischer Parameter und sonografischer Befunde kann eine in der klinischen Praxis gut anwendbare Kategorisierung in niedrige, mittlere und hohe Wahrscheinlichkeit für das Vorliegen einer simultanen Choledocholithiasis erfolgen ( $\triangleright$ Tab. 1). Zu berücksichtigen ist bei der Risikoklassifikation auch, dass bei Patienten mit symptomatischer Cholezystolithiasis die Prävalenz simultaner Gallengangsteine mit dem Alter zunimmt [181, 186, 221].

\section{EMPFEHLUNG II.8.}

Bei Patienten mit hoher Wahrscheinlichkeit für das Vorliegen einer Choledocholithiasis sollte primär die ERC in therapeutischer Intention erfolgen (B, I, starker Konsens).

Bei niedriger oder mittlerer Wahrscheinlichkeit für das Vorliegen einer Choledocholithiasis soll die ERC nicht zur Diagnose oder zum Ausschluss von Gallengangsteinen eingesetzt werden (A, I, starker Konsens).

Bei Patienten mit mittlerer Wahrscheinlichkeit für das Vorliegen einer Choledocholithiasis sollen eine Endosonografie oder eine MRCP durchgeführt und die Entscheidung zur Durchführung einer ERC in Abhängigkeit vom Ergebnis dieser Untersuchungen getroffen werden (A, I, starker Konsens, NKLM).

\section{Kommentar:}

Die ERC hat für die Choledocholithiasis eine sehr hohe Sensitivität und Spezifität von deutlich über $90 \%$ [197, 201, 222 - 226]. Andererseits handelt es sich um eine invasive Methode mit einer Komplikationsrate von etwa 5 bis $10 \%$. Die häufigste Komplikation ist die Post-ERC-Pankreatitis [227]. Da die Prävalenz von Gallengangsteinen bei Patienten mit Cholezystolithiasis altersabhängig zwischen 5 und $15 \%$ liegt $[228,229]$, würde eine generelle Empfehlung zum Ausschluss von Gallengangsteinen vor einer Cholezystektomie mittels ERC zu viele Patienten unnötig den Risiken dieses Verfahrens aussetzen. Daher ist ein an das Risiko für eine Choledocholithiasis adaptiertes Vorgehen sinnvoll [195, 211, 230 - 232].

Bei direktem sonografischen Nachweis von Gallengangsteinen und/oder bei Vorliegen einer akuten Cholangitis ist primär die ERC indiziert, da sie neben dem Nachweis von Gallengangsteinen die gleichzeitige therapeutische Intervention erlaubt (biliäre Sphinkterotomie und Steinextraktion). Die Steinextraktions- und Komplikationsraten sind zwischen der prä- und der intraoperativen ERC vergleichbar, aber die intraoperative ERC ist mit einer geringeren Inzidenz von ERC-bedingten Komplikationen und mit einem kürzeren Krankenhausaufenthalt, jedoch mit höherem logistischen Aufwand verbunden [233, 234].

Bei hochgradigem Verdacht auf eine Choledocholithiasis ohne direkten bildgebenden Nachweis von Gallengangsteinen ( $\triangleright$ Tab. 1) ist die primäre Durchführung einer ERC vertretbar. Es ist aber zu berücksichtigen, dass auch in der Hochrisikogruppe die durchschnittliche Prävalenz einer Choledocholithiasis nur zwischen 35 und $76 \%$ liegt [220, 235 -243]. In dieser Situation
- Tab. 1 Kriterien für eine simultane Choledocholithiasis bei Cholezystolithiasis.

\section{hohe Wahrscheinlichkeit einer simultanen Choledocholithiasis} (>50\%)

- sonografisch erweiterter extrahepatischer Gallengang (> 7mm) + Hyperbilirubinämie

+ Erhöhung von Y-GT, AP, ALT oder AST oder

- sonografischer Nachweis von Gallengangkonkrementen oder

- klinische und laborchemische Kriterien einer aszendierenden Cholangitis

mittlere Wahrscheinlichkeit einer simultanen Choledocholithiasis (5-50\%)

- keine Kriterien für hohe oder niedrige Wahrscheinlichkeit niedrige Wahrscheinlichkeit einer simultanen Choledocholithiasis (<5\%)

- Gallengang normal weit (bis $7 \mathrm{~mm}$ )

- Gesamtbilirubin, y-GT, AP, ALT bzw. AST während der aktuellen Schmerzepisode nicht erhöht

- Fehlen von Episoden mit biliärer Pankreatitis, acholischen Stühlen und/oder Urobilinogenurie bzw. Bilirubinurie in der aktuellen Vorgeschichte

können daher im Einzelfall weitere bildgebende Verfahren (insbesondere Endosonografie und MRCP) sinnvoll sein, um zur Choledocholithiasis alternative mögliche Ursachen für eine Gallengangerweiterung in Kombination mit der Erhöhung von biochemischen Leberfunktionsparametern oder rechtsseitigen Oberbauchschmerzen differenzialdiagnostisch abzugrenzen [244]. In einer prospektiven Studie konnte auch bei Patienten mit Hochrisikokriterien für das Vorliegen einer Choledocholithiasis nach Durchführung einer Endosonografie die Anzahl erforderlicher ERC um mehr als die Hälfte reduziert werden [241]. Die Endosonografie ermöglicht Aussagen zur Anzahl und Größe der Konkremente und kann so zur Planung der ERC beitragen [245]. Eine Metaanalyse konnte zeigen, dass die Endosonografie im Vergleich zum Goldstandard ERC eine sehr hohe Genauigkeit nicht nur für die Diagnose einer Choledocholithiasis, sondern auch für die Abklärung einer biliären Obstruktion anderer Ursache hat (gepoolte Sensitivität, Spezifität und Genauigkeit: 88, 90 bzw. 97\%) [246].

Das völlige Fehlen klinischer, biochemischer und sonografischer Prädiktoren schließt das Vorliegen einer Choledocholithiasis mit einer der ERC und der intraoperativen Cholangiografie vergleichbaren Sicherheit aus [200, 201, 204, 219]. Daher sind vor einer Cholezystektomie im Regelfall weder weiterführende bildgebende Verfahren noch eine ERC erforderlich.

Um die Indikation zu einer therapeutischen ERC beurteilen zu können, ist bei Patienten mit einer mittleren Wahrscheinlichkeit für eine Choledocholithiasis ( $\triangleright$ Tab. 1) die weitere diagnostische Abklärung sinnvoll [195, 231, 244, 247 - 250].

Von den dafür verfügbaren Methoden weist die Endosonografie die höchste Sensitivität für die Detektion von Steinen im Ductus hepatocholedochus auf. Sie ist risikoärmer als die diagnostische ERC [251] und besitzt bei entsprechender Untersucher- 
erfahrung eine nahezu $100 \%$ ige Sensitivität sowie eine hohe Spezifität (>93\%) zum Nachweis bzw. Ausschluss von Gallengangsteinen, insbesondere auch für kleinere ( $<5 \mathrm{~mm})$ und präpapilläre Konkremente [142, 143, 194, 222, 223, 231, 235, 244, 245, 247 -249, 252 - 268]. In einer 2008 publizierten Metaanalyse, die 27 Studien mit insgesamt 2673 Patienten mit Verdacht auf Choledocholithiasis einschloss, wurde für die Endosonografie eine gepoolte Sensitivität und Spezifität von 94 bzw. $97 \%$ ermittelt [269]. Die Ergebnisse der Endosonografie sind von der Steingröße und dem Gallengangdurchmesser unabhängig. In einer prospektiven Studie erwies sich die Endosonografie der ERC bei der Entdeckung von Steinen $<4 \mathrm{~mm}$ als überlegen [249]. Die mit longitudinalen Echoendoskopen sowie mit extraduktal eingesetzten radialen Minisonden erreichten diagnostischen Ergebnisse sind vergleichbar mit den für radiale Echoendoskope berichteten [143, 260, 270 - 276]. Diagnostische Limitationen ergeben sich insbesondere bei postoperativ veränderter Anatomie oder bei Stenosen des oberen Verdauungstraktes. Bei Patienten mit intermediärer Wahrscheinlichkeit von Gallengangsteinen kann durch den routinemäßigen Einsatz der Endosonografie (EUS first-Strategie) die Anzahl der erforderlichen ERC um etwa 2/3 reduziert werden [241, 247-250, 277].

Die MRCP weist insgesamt eine mit der Endosonografie vergleichbare mediane Sensitivität (77-100\%) und Spezifität (73-100\%) auf, wobei ihre Aussagefähigkeit für Konkremente $<5 \mathrm{~mm}$ und damit die Beurteilung einer Mikrolithiasis eingeschränkt ist $[185,226,231,244,256,258,262,263,268,278$ 309]. Weitere Einschränkungen betreffen Verfügbarkeit, Kosten und patientenindividuelle Faktoren. Ein systematisches Review (25 Studien, 2310 Patienten) kalkulierte eine gepoolte Sensitivität und Spezifität der MRCP von 90 bzw. 95 \% [310]. Mit einer MRCPfirst-Strategie bei Verdacht auf Choledocholithiasis können mindestens $50 \%$ der präoperativen ERC vermieden werden [311, 312]. Eine Metaanalyse [306] von fünf prospektiv-vergleichenden Studien [231, 244, 256, 258, 263] mit insgesamt 301 Patienten fand zwischen Endosonografie und MRCP keine signifikanten Unterschiede hinsichtlich der Sensitivität (93 vs. $85 \%$ ) und Spezifität (96 vs. $93 \%$ ) für die Diagnose von Gallengangsteinen. Auch ein Cochrane-Review, das insgesamt 18 Studien (darunter nur zwei direkt vergleichende) einbezog, konstatierte Gleichwertigkeit beider Methoden [268], während ein weiteres systematisches Review acht vergleichende Studien analysierte und für gleiche Prätest-Wahrscheinlichkeiten eine höhere Nachweis- und Ausschluss-Wahrscheinlichkeit der Endosonografie für das Vorliegen einer Choledocholithiasis ermittelte [309]. In einer prospektiv kontrollierten Studie wurde auch über eine tendenziell höhere Spezifität der Endosonografie (95\%) im Vergleich zur MRCP (73\%) berichtet [256]. Eine jüngst publizierte Metaanalyse von 5 direkt vergleichenden prospektiven Kohortenstudien ermittelte dagegen bei gleicher Spezifität beider Methoden (90 vs. $92 \%$ ) eine signifikant höhere Sensitivität und diagnostische Odds Ratio (97 bzw. 162,5\%) für die Endosonografie im Vergleich zur MRCP (87 bzw. 79\%) [313].

Die Spiral-Computertomografie weist eine Sensitivität von 65 - $92 \%$ und eine Spezifität von 73-97\% für die Diagnose einer Choledocholithiasis auf [277, 289, 314, 315]. Eine höhere und annähernd der MRCP vergleichbare Genauigkeit für die Detektion von Gallengangsteinen hat die CT-Cholangiografie [257, 263, 289, 316]. Wobei falsch negative Fälle insbesondere bei Gallengangsteinen $<5 \mathrm{~mm}$ beschrieben worden sind [263, 277]. Aufgrund der damit verbundenen Strahlenexposition sowie der hohen Rate an Nebenwirkungen bezüglich der Leber-, Nierenund Herz-Kreislauffunktion bei gleichzeitig vorhandenen besseren und nebenwirkungsärmeren Verfahren wie Endosonografie und MRCP hat die CT-Cholangiografie mit intravenöser Kurzinfusion heute jedoch keinen klinischen Stellenwert für die Diagnostik bei Verdacht auf Choledocholithiasis.

Zur Darstellung der Anatomie und/oder zum Nachweis von Gallengangsteinen können auch die intraoperative Cholangiografie und die laparoskopische Sonografie durchgeführt werden [317 - 319]. In einer Metaanalyse, in die 11 vergleichende Studien eingeschlossen wurden, hatten die intraoperative Cholangiografie als auch die laparoskopische Sonografie jeweils eine gepoolte Sensitivität von $87 \%$ sowie eine gepoolte Spezifität von 99 bzw. $100 \%$ [320]. Aufgrund der begrenzten Verfügbarkeit und Expertise und des damit verbundenen zeitlichen und logistischen Aufwandes wird der selektive Einsatz beider Methoden in Deutschland derzeit nicht empfohlen [321-324].

Für die Diagnostik von symptomatischen Patienten nach Cholezystektomie und für die Patientenselektion zur ERC durch Anwendung weniger invasiver Methoden liegen kaum valide Daten vor. Nach Cholezystektomie wird ohne Vorliegen von Gangsteinen oder anderer biliärer Pathologien eine geringe Zunahme der Gallengangweite beschrieben [212, 213, 325 - 328]. Da nur in einem sehr geringen Prozentsatz der untersuchten cholezystektomierten Patienten der für Patienten mit in situ befindlicher Gallenblase allgemein akzeptierte obere Grenzwert für die Gallengangweite von $7 \mathrm{~mm}$ überschritten wird, kann dieser auch auf die postoperative Situation übertragen werden [214, 215, 328]. Allerdings ist bei Patienten mit einer Gallengangweite bis $10 \mathrm{~mm}$ nach Cholezystektomie das Vorliegen einer Choledocholithiasis wenig wahrscheinlich, wenn keine weiteren klinischen oder laborchemischen Kriterien vorliegen. Sowohl die Endosonografie als auch die MRCP sind bei Patienten mit Cholezystektomie und biliären Beschwerden geeignet, mit hoher Genauigkeit eine Choledocholithiasis oder alternative biliopankreatische Ursachen zu detektieren bzw. auszuschließen [255, 329-332]. Insofern kann das für Patienten mit Gallenblase in situ und Verdacht auf Choledocholithiasis empfohlene risikoadaptierte Vorgehen grundsätzlich auch auf cholezystektomierte Patienten übertragen werden.

EMPFEHLUNG II.9.

Bei Verdacht auf eine akute Cholangitis sollen

a) klinische Symptome einer systemischen Inflammation (Fieber, Schüttelfrost) und laborchemische Entzündungsparameter (Leukozyten, CRP) evaluiert,

b) Cholestase-Parameter (Bilirubin, AP, Y-GT und Transaminasen) bestimmt werden und

c) transabdominell-sonografisch die Gallengänge (Gallengangweite $>7 \mathrm{~mm}$ oder Nachweis eines Konkrementes oder eines anderen Abflusshindernisses) abgeklärt werden. 
Bei Vorliegen pathologischer Befunde aller drei diagnostischen Kategorien $(a+b+c)$ liegt mit sehr hoher Wahrscheinlichkeit eine obstruktive Cholangitis vor (A, II, starker Konsens).

Lassen sich sonografisch weder eine Gallengangerweiterung noch ein Gallengangkonkrement oder ein anderes Abflusshindernis nachweisen (Kriterium c), sollten eine Endosonografie oder MRCP durchgeführt werden (B, I, starker Konsens).

\section{Kommentar:}

Die akute Cholangitis ist wegen der erhöhten Letalitätsrate (in seit dem Jahr 2000 publizierten Studien zwischen 3 - 10 \% [333]) eine gefürchtete Komplikation der Choledocholithiasis. Sie entsteht als Resultat einer biliären Abflussstörung und einer Infektion. Häufigste Ursache der biliären Abflussstörung ist mit $28-70$ \% der Fälle die Choledocholithiasis, gefolgt von malignen Stenosen, benignen Stenosen und selteneren Ursachen wie intraduktalen Parasiten [333]. Die klinische Präsentation der akuten Cholangitis ist nicht immer typisch. Eine Charcot-Trias wurde in verschiedenen Studien in nur 16 bis maximal 72 \% der Fälle beobachtet [334 - 339]. Nach den Diagnosekriterien der Tokyo-Leitlinie 2018 ist die Diagnose der Cholangitis gesichert, wenn systemische Infektionszeichen (klinisch Fieber; laborchemisch: Leukozytose oder Leukozytopenie, CRP-Erhöhung), ein Verschlussikterus oder erhöhte Cholestase-Parameter im Blut sowie sonografisch ein erweiterter Gallengang bzw. eine Cholangiolithiasis oder ein anderer pathologischer Befund an den Gallengängen (z. B. Stenose oder Stent) vorliegen [339]. Ein Verdacht auf Cholangitis ergibt sich, wenn neben den systemischen Infektzeichen noch ein zweiter Parameter erfüllt ist (entweder Verschlussikterus bzw. erhöhte Cholestase-Parameter oder ein erweiterter Gallengang bzw. pathologischer Gallengangbefund) [336, 339]. Diese Diagnosekriterien erreichten in zwei retrospektiven asiatischen Studien eine hohe diagnostische Genauigkeit und erwiesen sich im Vergleich zur Charcot-Trias als signifikant überlegen [337, 339].

Die transabdominelle Sonografie stellt bei Patienten mit dem Verdacht auf eine akute Cholangitis das primäre bildgebende Verfahren dar und sollte aufgrund ihrer flächendeckenden bettseitigen Verfügbarkeit, fehlenden Strahlenbelastung und Wiederholbarkeit bei allen Patienten mit dieser Verdachtsdiagnose bei der primären Präsentation und im Verlauf eingesetzt werden [340 342]. Die transabdominelle Sonografie hat in diesem Zusammenhang auch bei notfallmäßiger Durchführung eine hohe Aussagekraft $[343,344]$. Trotz Limitationen in der Detektion kleiner Cholesterolkonkremente kann auch die Computertomografie zur Diagnose einer akuten Cholangitis beitragen. Computertomografische Cholangitiskriterien sind ein transientes inhomogenes Enhancement des Leberparenchyms in der arteriellen Phase und ein peribiliäres Ödem [336, 339, 340, 345 - 349]. Bei der bildgebenden Abklärung des Verdachts auf eine akute Cholangitis sollten das Vorliegen einer Obstruktion mit konsekutiver Gallengangerweiterung, die Höhe der Obstruktion innerhalb des Gangsystems, eine Aerobilie, eine mögliche Wandverdickung des extrahepatischen Gallengangs, ein peribiliäres Ödem, sowie die
Ursache der Obstruktion wie Gallen(gang)steine oder Strikturen evaluiert und beschrieben werden. Nach möglichen Komplikationen wie Leberabszessen oder Pfortaderthrombosen sollte gezielt gesucht werden [340, 341, 349, 350].

Endosonografie und MRCP können bei nicht konklusivem Sonografiebefund vor Durchführung einer ERC bei akuter Cholangitis zur Klärung der Ätiologie eingesetzt werden, dürfen aber nicht eine dringliche ERC verzögern. Auf den hohen Stellenwert beider Methoden zur Diagnostik einer Choledocholithiasis und zur Klärung der Ursache einer biliären Obstruktion wurde bereits unter II.9 hingewiesen. Typische endosonografische Kriterien einer akuten Cholangitis sind eine diffuse konzentrische Wandverdickung, ein echoarmer peribiliärer Randsaum von mehr als 15 mm Länge sowie der Nachweis heterogenen, nicht echofreien Ganginhalts ohne Schallschatten [351]. Als magnetresonanztomografische Kriterien einer akuten Cholangitis wurden eine erhöhte periduktale Signalintensität im T2-gewichteten Bild, eine transiente periduktale Signalabschwächung sowie eine Wandverdickung der extrahepatischen Gallengänge beschrieben [352]. Eine dringlich erforderliche ERC darf durch diese diagnostischen Maßnahmen nicht verzögert werden. Bei mäßig schwerer und bei schwerer Cholangitis verschlechtert die Verzögerung einer dringlichen ERC die Prognose und erhöht die Kosten [353, 354].

EMPFEHLUNG II.10.

Der Schweregrad einer akuten Cholangitis sollte bei Erstkontakt und abhängig vom Krankheitsverlauf (zumindest ein weiteres Mal innerhalb der ersten 24 Stunden) beurteilt und dokumentiert werden.

Zur Beurteilung des Schweregrads der akuten Cholangitis sollten neben der allgemeinen klinischen Einschätzung Bewusstseinslage, Kreislaufparameter (Herzfrequenz und Blutdruck), Sauerstoffsättigung und Laborparameter (insbesondere Thrombozyten, Kreatinin/glomeruläre Filtrationsrate, Quick/ INR, Albumin) herangezogen werden (B, III, starker Konsens).

\section{Kommentar:}

Der Schweregrad der akuten Cholangitis bestimmt maßgeblich die Indikation zur intensivmedizinischen Therapie sowie Notwendigkeit und Zeitpunkt einer frühzeitigen biliären Drainage [342]. In den aktuellen Tokyo-Leitlinien [339] wird der Schweregrad der Cholangitis in drei Grade eingeteilt. Der höchste Schweregrad (Grad III) ist bei Schock mit Katecholamin-Pflichtigkeit, Bewusstseinstrübung oder Versagen von Lunge, Niere, Leber oder Gerinnung gegeben. Für die Diagnose einer mäßig schweren Verlaufsform (Grad II) ist der Nachweis von mindestens zwei der folgenden Kriterien erforderlich: (1) Leukozytose > $12 \mathrm{G} / \mathrm{l}$ oder Leukopenie $<4 \mathrm{G} / \mathrm{l}$, (2) Fieber $\geq 39^{\circ} \mathrm{C}$, (3) Alter $\geq 75$ Jahre, (4) Gesamtbilirubin $\geq 5 \mathrm{mg} / \mathrm{dl}(103 \mu \mathrm{mol} / \mathrm{l})$ und (5) Albumin $<25 \mathrm{~g} / \mathrm{l}$ [333, 339]. Sie kann sich insbesondere bei unzureichender biliärer Drainage zu einer schweren Verlaufsform mit ungünstiger Prognose entwickeln. Neben einer verzögerten oder ineffektiven interventionellen Therapie wurden in Studien als Risikofaktoren für die Entwicklung eines schweren Verlaufes identifiziert: hohes Alter, 
schwere Komorbidität, andere Ätiologie als Gallengangsteine bzw. maligne Gallengangobstruktion, Cholangitis mit Leberabszess, Leberzirrhose, hiläre und intrahepatische Gallengangobstruktion, Antibiotikaresistenz der Erreger bzw. nicht adäquate kalkulierte Antibiotikabehandlung [337, 355 - 359].

\section{EMPFEHLUNG II.11}

Bei Patienten mit akuter Pankreatitis, bei denen in der primären Bildgebung Gallengangsteine nachgewiesen werden oder komplizierend ein Gallengangverschluss oder eine Cholangitis vorliegen, soll primär eine ERC mit therapeutischer Zielstellung erfolgen.

In allen anderen Fällen sollen primär eine Endosonografie oder eine MRCP durchgeführt werden, um die Indikation zur ERC zu klären (selektive ERC-Strategie) (A, II, starker Konsens, NKLM).

\section{Kommentar:}

In westlichen Industriestaaten wird bei 35 bis $60 \%$ der Patienten mit akuter Pankreatitis eine biliäre Ursache nachgewiesen. Frauen sind deutlich häufiger betroffen als Männer, und die Inzidenz steigt mit zunehmendem Lebensalter an [360 - 364]. Neben dem Fehlen anamnestischer und klinischer Hinweise auf eine alternative Genese (insbesondere Alkoholabusus), sind die Anamnese einer Cholezystektomie, der Nachweis von Gallenblasensteinen und/oder Gallengangsteinen im transabdominellen Ultraschall, eines (transienten) Anstiegs der ALT oder AST auf das 2 3-fache der oberen Norm sowie eine primäre Erhöhung bzw. ein Anstieg des Serumbilirubins im Verlauf starke Prädiktoren für die biliäre Genese einer akuten Pankreatitis. Der Nachweis von Gallenblasensteinen oder Sludge in Kombination mit einer Erhöhung von Leberfunktionsparametern hat einen sehr hohen positiven Voraussagewert für die biliäre Genese einer akuten Pankreatitis. Insbesondere der Nachweis zahlreicher kleiner Gallenblasensteine spricht für eine biliäre Pankreatitis [365]. Darüber hinaus steigt die Wahrscheinlichkeit für eine biliäre Ursache mit zunehmendem Lebensalter und ist bei Frauen deutlich höher als bei Männern [366 - 379]. Allerdings demonstrierte eine prospektive Studie, dass bei $12 \%$ aller Patienten mit akuter biliärer Pankreatitis die ALT normal sein kann und bei weiteren $17 \%$ die Erhöhung geringer als auf das 3-fache der Norm ausfallen kann [380]. Eine prospektiv-multizentrische, ERC-kontrollierte Studie zeigte auf, dass in der frühen Phase einer biliären Pankreatitis biochemische und sonografische Prädiktoren keinen (sonografischer Nachweis von Gallengangsteinen oder Gangerweiterung; Erhöhung von Bilirubin oder Transaminasen) oder nur einen geringen (Erhöhung von AP oder Y-GT) Voraussagewert für das Vorliegen von Gallengangsteinen hatten [216]. Wenngleich Studien zeigen konnten, dass bei akuter alkoholtoxischer Pankreatitis das Verhältnis von Serumlipase zu Serumamylase signifikant höher war als bei nicht-alkoholischer und biliärer Genese [381 - 383], erlaubt die Lipase-Amylase-Ratio keine ausreichend sichere Differenzierung zwischen akuter biliärer und alkoholischer Pankreatitis [384, 385].
Normal weite Gallengänge und der fehlende Nachweis von Gallengangsteinen in der transabdominellen Sonografie schließen die biliäre Genese einer akuten Pankreatitis nicht aus [223, 226, 376, 379, 386, 387]. Der Spontanabgang von Konkrementen im frühen Verlauf einer akuten biliären Pankreatitis ist häufig. Nur in etwa $18-45 \%$ der Fälle werden persistierende Gallengangsteine durch Endosonografie, MRCP oder ERC nachgewiesen [197, 223, 374, 378, 386 - 391]. Ein Anstieg von Transaminasen und Pankreasenzymen im Serum, die Persistenz erhöhter Werte und ein erhöhtes Serumbilirubin am zweiten Krankenhaustag wurden als Kriterien für die Persistenz von Gallengangsteinen identifiziert, während ein Abfall Hinweis auf eine Steinpassage ist [374, 388, 392].

Die diagnostische Genauigkeit des transabdominellen Ultraschalls und der CT zur Detektion von persistierenden Gallengangsteinen bei akuter biliärer Pankreatitis ist limitiert [223, 226, 386, 387]. Eine kurzfristige Wiederholung der transabdominellen Sonografie ist sinnvoll und kann deren Genauigkeit signifikant steigern [393].

Ist durch transabdominelle Sonografie eine definitive Klärung nicht möglich, kann diese durch MRCP und Endosonografie erfolgen. Welches der beiden Verfahren zum Einsatz kommt, soll unter Berücksichtigung von Verfügbarkeit, lokaler Expertise und patientenindividuellen Faktoren entschieden werden. Beide Methoden sind bei deutlich geringerer Invasivität ähnlich sensitiv und spezifisch wie die ERC [197, 222, 223, 226, 378, 387, 389, 390, $394-$ 397]

Ein systematisches Review der Daten von Patienten mit Verdacht auf biliäre Pankreatitis aus sechs prospektiven [197, 222, 223, 389, 396, 397] und einer retrospektiven Studie [398] machte deutlich, dass durch endosonografische Selektion von Patienten mit Choledocholithiasis zur therapeutischen ERC in den Studienkollektiven kumulativ $71 \%$ aller sonst erforderlichen ERC einschließlich der damit verbundenen Komplikationen vermieden werden konnten [399]. Ähnliche Raten vermiedener ERC wurden für den primären Einsatz der MRCP bei biliärer Pankreatitis kalkuliert $[400,401]$. Studien, die die Effektivität von Endosonografie und MRCP bei akuter biliärer Pankreatitis vergleichen, sind nicht verfügbar. Indirekte Evidenz spricht für eine etwas höhere Effektivität der Endosonografie. In einer retrospektiven Studie, in die 223 Patienten mit einer milden akuten biliären Pankreatitis eingeschlossen waren, konnte in 14 von 33 (42\%) Fällen mit negativem Ergebnis der konventionellen radiologischen Bildgebung (transabdominelle Sonografie, CT, in fast 50 \% der Fälle auch MRCP) erst eine zusätzliche Endosonografie die biliäre Ursache detektieren [402]. Zwei weitere retrospektive Analysen zeigten eine mit jeweils $62 \%$ relativ geringe diagnostische Sensitivität der MRCP bei akuter biliärer Pankreatitis [391, 393].

Eine Entscheidungsbaumanalyse verglich eine durch MRCP oder Endosonografie gesteuerte Selektion von Patienten mit akuter biliärer Pankreatitis zur therapeutischen ERC mit einer selektiven ERC-Strategie (primär konservatives Vorgehen bei milder Pankreatitis, primäre ERC mit Sphinkterotomie bei schwerer Pankreatitis). Das endosonografisch gesteuerte Vorgehen erwies sich insbesondere bei schwerer akuter Pankreatitis in Bezug auf Kosten, Vermeidung von ERC und Komplikationen als die vorteilhafteste Strategie [403]. Zu einem ähnlichen Ergebnis kam eine weitere Entscheidungsbaumanalyse: Bei akuter biliärer Pankreatitis 
mit einer Wahrscheinlichkeit des Vorliegens von Gallengangsteinen von 7 - $45 \%$ ist die Endosonografie gefolgt von einer therapeutischen ERC bei Nachweis von Gallengangsteinen oder bei biliärer Sepsis die kosteneffektivste Strategie. Bei einer höheren Wahrscheinlichkeit ist dagegen die primäre Durchführung einer ERC sinnvoll [230]. Dies betrifft insbesondere Patienten mit Cholangitis oder einer persistierenden Cholestasekonstellation, d. h. Verschlussikterus, persistierender Erhöhung von Cholestase-Enzymen und/oder Erweiterung intra- und extrahepatischer Gallengänge [404]. Bei Patienten mit akuter biliärer Pankreatitis und begleitender Cholangitis reduziert die frühe Durchführung einer ERC nach den Ergebnissen einer Cochrane-Analyse signifikant sowohl die lokalen und systemischen Pankreatitiskomplikationen als auch die Letalität. Auch bei Patienten mit begleitender biliärer Obstruktion scheint die frühe ERC die Pankreatitiskomplikationen zu reduzieren, ohne allerdings Einfluss auf die Letalität zu haben [405]. Metaanalysen und Leitlinien ergeben kein einheitliches Bild zum Stellenwert der ERC bei voraussichtlich schwerem Verlauf einer akuten biliären Pankreatitis, während bei voraussichtlich mildem Verlauf 7 von 8 Metaanalysen gegen die Durchführung einer primären ERC sprechen [404, 405].

\section{EMPFEHLUNG II.12.}

Ist nach Anamnese, klinischer Untersuchung, Labordiagnostik und transabdominellem Ultraschall die Ursache einer akuten Pankreatitis nicht zu klären, sollen eine Endosonografie oder alternativ eine MRCP erfolgen (A, II, starker Konsens).

\section{Kommentar:}

Die Ätiologie der akuten Pankreatitis kann in der Mehrheit der Fälle geklärt werden. Gallengangsteine und Alkoholabusus sind die häufigsten Ursachen, in einigen epidemiologischen Studien wird aber über einen hohen, tendenziell abnehmenden Anteil an idiopathischer Pankreatitis berichtet [361, 362, 364]. Die relative Häufigkeit ätiologisch unklarer Pankreatitiden hängt von der Intensität der primären Diagnostik ab. Nach primärer klinischanamnestischer, laborchemischer (Leberfunktionsparameter, Serumkalzium, Triglyzeride) und bildgebender (Abdomensonografie und/oder Computertomografie) Diagnostik bleiben etwa 10 $30 \%$ aller akuten Pankreatitisfälle ätiologisch unklar. Rezidive sind bei Patienten mit ätiologisch unklarer Pankreatitis häufig (20-50\%) und gehen mit einer relevanten Letalität einher [406 - 409]. Eine gezielte und intensive Diagnostik vermag bis zu $80 \%$ aller initial ätiologisch unklaren Fälle zu klären und ermöglicht durch gezielte Therapie die Verminderung der Rezidivrate [410 - 414]. Die häufigsten Ursachen sind biliärer Natur (Mikrolithiasis bzw. Sludge der Gallenblase, Choledocholithiasis, andere benigne und maligne biliopankreatische Abflussstörungen, biliopankreatische Ganganomalien). Daher hat die ERC ggf. in Kombination mit einer Galleaspiration bei idiopathischer akuter Pankreatitis einen hohen diagnostischen Ertrag [410, 413, 415, 416], allerdings um den Preis einer signifikanten Komplikationsrate. In der größten publizierten Serie wurden Komplikationen eines ERC-gestützten Vorgehens in 11,5\% der 1241 Fälle beschrieben
[416]. Daher wurden weniger invasive Strategien unter Einschluss der transabdominellen Sonografie, der Endosonografie und der MRCP evaluiert.

Die transabdominelle Sonografie vermag Sludge und Mikrolithen (d.h. Konkremente bis maximal $3 \mathrm{~mm}$ ) in der Gallenblase nur mit mäßiger Sensitivität zu verifizieren. Die Sensitivität lässt sich durch Einsatz von Harmonic imaging verbessern [417 - 419]. Bei Patienten mit einer operativ bestätigten Mikrolithiasis, die transabdominell sonografisch nicht detektierbar war, konnte in mehreren Untersuchungen eine sehr hohe Sensitivität und Spezifität der Endosonografie nachgewiesen werden [142, 378, 420, 421]. Der diagnostische Ertrag der Endosonografie bei idiopathischer akuter Pankreatitis (einschließlich von Fällen mit rezidivierender idiopathischer Pankreatitis) wird in Studien zwischen 41 und $84 \%$ angegeben. Dabei stehen der Nachweis von Sludge und Mikrolithiasis von Gallenblase und Gallengängen ganz im Vordergrund (14-61\%), gefolgt von pankreatobiliären Neoplasien ( $0-16 \%)$ und Ganganomalien (0 - 7\%). Relativ häufig finden sich bei diesen Patienten auch endosonografische Kriterien der chronischen Pankreatitis (2 - 45\%). Der diagnostische Ertrag der Endosonografie ist deutlich höher bei Patienten mit Gallenblase in situ als bei Zustand nach Cholezystektomie [411, 414, 422 - 431].

Durch MRCP ohne oder mit Sekretinstimulation können insbesondere funktionelle oder morphologische Abflussstörungen der Major- und Minorpapille sowie pankreatobiliäre Ganganomalien als mögliche Ursachen einer idiopathischen akuten Pankreatitis identifiziert werden [414, 426, 429, 432].

Welches der beiden Verfahren (Endosonografie vs. MRCP) primär zum Einsatz kommt, soll unter Berücksichtigung von Verfügbarkeit, lokaler Expertise und patientenindividuellen Faktoren entschieden werden. Wenngleich in vergleichenden Studien der diagnostische Ertrag der MRCP jeweils geringer war als der der Endosonografie, wurde dennoch durch MRCP jeweils ein geringer diagnostischer Zusatzgewinn zum Ergebnis der Endosonografie erzielt [414, 426, 429]. Daher bietet sich unter Berücksichtigung der Ergebnisse eines systematischen Reviews [433] nach ergebnisloser ätiologischer Primärdiagnostik bei Patienten mit idiopathischer akuter Pankreatitis zunächst die Durchführung einer Endosonografie an, die bei negativem Ergebnis durch eine MRCP ergänzt wird. Weitere spezielle diagnostische Verfahren (genetische Testung, sekretinstimulierte Endosonografie, intraduktale Sonografie) bleiben Einzelfällen vorbehalten [413, 426, 434].

EMPFEHLUNG II.13.

Zur medikamentösen Prophylaxe einer post-ERC-Pankreatitis soll $100 \mathrm{mg}$ Diclofenac oder $100 \mathrm{mg}$ Indometacin rektal als Suppositorium vor oder unmittelbar nach der ERC appliziert werden (A, I, Konsens).

Bei erhöhtem Risiko für eine PEP sollte zusätzlich zu dieser Prophylaxe die passagere Einlage eines Pankreasgangstents in Erwägung gezogen werden ( $\mathrm{B}, \mathrm{I}$, Konsens). 


\section{Kommentar:}

Die Empfehlung wurde von der S2k-Leitlinie Qualitätsanforderungen in der gastrointestinalen Endoskopie übernommen [435]. Zur medikamentösen Prophylaxe einer PEP ist die rektale Gabe von Diclofenac oder Indomethacin in vergleichenden Studien evidenzbasiert [436 -441]. Die rektale Applikation von $100 \mathrm{mg}$ Diclofenac oder Indometacin unmittelbar vor oder nach der ERC mit Papillotomie reduzierte die Inzidenz der PEP signifikant und soll daher standardgemäß angewandt werden. In der europäischen Leitlinie [442] wird auf der Basis von Metaanalysen weiterer prospektiv-randomisierter Studien [443 - 448] die routinemäßige Anwendung der o. g. NSAR bei allen ERC von Patienten ohne Kontraindikationen dringend empfohlen. Die Number needed to treat (NNT) liegt dabei zwischen 11 und 20.

Weitere Maßnahmen zur Reduktion des Pankreatitisrisikos sind die drahtgeführte Kanülierungstechnik, die Vermeidung der Kontrastmittelfüllung des Pankreasgangs und die passagere Einlage eines Pankreasgangstents [435]. Bezüglich der Einlage eines prophylaktischen Pankreasgangstents bei erhöhtem Risiko für PEP zeigten Metaanalysen eine signifikante Reduktion der PEP durch das prophylaktische Pankreasgangstenting mit einer NNT von 8 [449 - 451]. Diese Technik führte bei Patienten mit hohem wie mittlerem Risiko zu einer Reduktion der PEP (RR 0,32, 95 \% Konfidenzintervall 0,19-0,52, P<0,001) [450]. Die Subgruppenanalyse der Studien von Elmunzer et al. [444] zur PEP-Prophylaxe mittels Indometacin ergab bei einer Stratifizierung der Patienten nach zusätzlichem Pankreasgangstent vs. kein Stent keinen additiven Effekt über die NSAR-Gabe hinaus an. Dies bestätigt auch eine andere Analyse [452]. Die Implantation einer Pankreasgangprothese zur PEP-Prophylaxe sollte bei Risikokonstellationen wie langwierigen Papillenkanülierungen, Precut-Sphinkterotomie oder Kanülierung des Gallengangs über einen einliegenden Draht im Pankreasgang erfolgen [453]. Zu beachten sind die Verwendung kleinlumiger Pankreasgangstents und die frühzeitige Entfernung des Stents nach 3-5 Tagen, um Pankreasgangschäden zu vermeiden [453 - 455]. Der Pankreasgangstent sollte mindestens 12 - $24 \mathrm{~h}$ in situ bleiben [442]. Studien zeigen, dass 5 Fr-Plastikstents effektiver als $3 \mathrm{Fr}$-Stents sind [456].

\section{EMPFEHLUNG II.14.}

Bei biliärer Indikation können Endosonografie und ERC unmittelbar nacheinander erfolgen, sofern eine endoskopische Therapie indiziert ist ( 0 , III, Konsens).

\section{Kommentar:}

Mehrere Studien haben zeigen können, dass die Durchführung von Endosonografie und ERC in einer Sitzung ohne ein höheres Komplikationsrisiko möglich und kosteneffektiv ist [457-465]. In einer retrospektiven Studie konnten biliäre Komplikationen im Intervall zwischen Endosonografie und ERC durch die kombinierte Durchführung in einer Sitzung (0\%) im Vergleich zur getrennten Durchführung (14\%) effektiv vermieden werden [463].

Erfolgt aufgrund logistischer Gegebenheiten oder patientenindividueller Faktoren eine Durchführung in getrennten Sitzungen, sollte zur Vermeidung biliopankreatischer Komplikationen das Intervall zwischen beiden Prozeduren so kurz wie möglich sein, da die Wahrscheinlichkeit einer Cholangitis und eines Pankreatitisrezidivs mit zunehmendem Intervall zwischen Diagnose und endoskopischer Gangsanierung sowie Cholezystektomie ansteigt [466 - 470]. Studien zu verschieden langen Zeitintervallen liegen jedoch nicht vor, sodass der Einsatz der Verfahren im klinischen Alltag nach deren Verfügbarkeit variiert. Erste Erfahrungen zeigen darüber hinaus, dass nach Scheitern einer therapeutischen ERC in gleicher Sitzung eine Endosonografie-gestützte Gallengangintervention effektiv und sicher durchgeführt werden kann [471, 472]. Die mögliche Kombination von Endosonografie und endoskopischer Gallengangintervention in einer Sitzung sollte bei der Patientenaufklärung berücksichtigt werden.

\section{EMPFEHLUNG II.15.}

Bei Verdacht auf intrahepatische Gallensteine ist die transkutane Sonografie die diagnostische Methode der ersten und die MRCP die Methode der zweiten Wahl (0, III, Konsens).

\section{Kommentar:}

Die Sonografie hat gegenüber der diagnostisch intendierten ERC Vorteile, da bei dieser lithogene Abbrüche der intrahepatischen Gallengänge durch die meist nicht kalzifizierten Konkremente in aller Regel nicht in Erscheinung treten. Darüber hinaus birgt die ERC die Gefahr der Cholangitisinduktion, sodass nicht invasive Verfahren zur Diagnostik vorzuziehen sind. Die Sonografie erlaubt zuverlässig die Darstellung der Steine und die Diagnose prästenotischer Erweiterungen der Gallengänge. Die MRCP ist ebenfalls der ERC bei der Diagnostik der intrahepatischen Steine überlegen (Sensitivität 97 vs. 59\%); auch intrahepatische biliäre Strikturen werden zuverlässig entdeckt (Spezifität $97 \%$, Sensitivität $93 \%$ ) [298, 473, 474]. Dabei konnte eine zusätzlich zur Standard-MRCP durchgeführte T1-gewichtete Untersuchung die Detektion und Differenzierung von Hepatolithiasis verbessern [475].

Mittels CT können dilatierte Gänge und Strikturen sowie Leberabszesse dargestellt werden, obgleich die Steine häufig nicht direkt zur Darstellung kommen [474]. Trotz dieser bekannten Limitationen der CT ist sie in einer aktuellen japanischen Studie, die die Erfahrungen über einen Zeitraum von 40 Jahren in Japan zusammenfasst, das mit $61 \%$ am häufigsten eingesetzte bildgebende Verfahren [476].

Die ERC und PTC haben ihre primäre Bedeutung für die anschlieBende Therapieplanung. Asymptomatische intrahepatische Steine werden im Verlauf von 15 Jahren nur bei 11,5\% der Patienten im Mittel nach 3,4 Jahren symptomatisch. Die häufigsten Symptome sind dann Koliken, Cholangitis und Leberabszess [477]. Aus diesem Grund ist das abwartende Verhalten gerechtfertigt. Bei symptomatischen Steinen ist die interdisziplinäre Therapieplanung sinnvoll [478]. Der Langzeiterfolg der Chirurgie ist insbesondere dann gegeben, wenn der Befall auf einzelne periphere Lebersegmente und einen Leberlappen begrenzt ist [479]. Bei diffuser Verteilung der intrahepatischen Steine sind modifizierte perkutan-transhepatische 
cholangioskopische Verfahren mit Lithotripsie und Gallengangdilatationen sinnvoll [474, $480-483]$.

\section{Therapie}

\section{IIIA. Konservative Therapie}

\section{EMPFEHLUNG IIIA. 1.}

Die medikamentöse Litholyse mit Ursodeoxycholsäure soll nur in Einzelfällen bei symptomatischen Patienten mit kleinen, mutmaßlich aus Cholesterin bestehenden Steinen oder Gallenblasen-Sludge durchgeführt werden; zuvor soll über die Möglichkeit der kurativen Cholezystektomie aufgeklärt werden (A, I, starker Konsens, NKLM).

\section{Kommentar:}

Der Patient muss vor der medikamentösen Litholyse auf das hohe Rezidivsteinrisiko aufmerksam gemacht werden. Die Metaanalyse von Studien zur Litholyse mit UDCA [484] ergab akzeptable Therapieerfolge (Steinfreiheit bis zu $60 \%$ nach 6 Monaten) vor allem bei Patienten mit einer funktionstüchtigen Gallenblase und multiplen kleinen nicht kalkhaltigen (röntgen-negativen) Cholesterinsteinen $<5(-10) \mathrm{mm}$.

Eine funktionstüchtige Gallenblase mit durchgängigem Ductus cysticus ist die Voraussetzung für die medikamentöse Litholyse [485]. Dies kann durch eine Sonografie mit Reizmahlzeit (Ejektionsfraktion $\geq 60 \%$ ) geprüft werden [486]. Als UDCA-Dosis werden mindestens $10 \mathrm{mg} / \mathrm{kg} / \mathrm{Tag}$ empfohlen [487]. Die Therapie sollte nach sonografisch verifizierter Steinfreiheit noch für $3 \mathrm{Mo-}$ nate fortgesetzt werden. Bei neuerlichen Symptomen nach erfolgreicher Therapie wird eine Sonografie durchgeführt. Bei symptomatischem Steinrezidiv ist in der Regel die Cholezystektomie indiziert. Bei asymptomatischen Patienten ist der Nutzen von Kontrolluntersuchungen nicht belegt.

\section{EMPFEHLUNG IIIA.2.}

Die extrakorporale Stoßwellenlithotripsie (ESWL) von Gallenblasensteinen wird nicht empfohlen (A, II, starker Konsens, NKLM).

\section{Kommentar:}

Die Negativempfehlung resultiert aus dem hohen Rezidivsteinrisiko. Mehrere Studien belegen die hohe Rezidivrate im Langzeitverlauf (25 - 64\% nach 5 Jahren und $54-80 \%$ nach 10 Jahren) [101, 488 - 497]. Gute Gallenblasenkontraktilität, BMI <25 kg/m² und UDCA-Prophylaxe verzögern die Rezidivsteinbildung, verhindern sie jedoch nicht; Patienten mit Solitärsteinen bekommen seltener Rezidive [488-492, 498 - 501]. Die Mehrzahl der Rezidivsteine ist symptomatisch und ein Drittel der Patienten muss im Mittel nach 3 Jahren cholezystektomiert werden [502].

Die direkte Litholyse mit Methyl-Ter-Butyl-Ether (MTBE) nach Punktion der Gallenblase ist obsolet.

\section{EMPFEHLUNG IIIA.3.}

Die medikamentöse Therapie der biliären Kolik sollte mit nichtsteroidalen Antiphlogistika (z. B. Diclofenac, Indometacin) erfolgen (B, I, starker Konsens). Zusätzlich können Spasmolytika (z. B. N-Butylscopolamin) oder Nitroglyzerin und bei starken Schmerzen Opioide (z. B. Buprenorphin, Pethidin) eingesetzt werden (0, IV, starker Konsens, NKLM).

\section{Kommentar:}

Bei der Behandlung der akuten Gallenkolik muss zwischen der unmittelbar erforderlichen medikamentösen Schmerztherapie und der kausalen Therapie (siehe IIIB.1) unterschieden werden. Allgemein ist zur Hemmung der Motilität der Gallenwege zunächst Nahrungskarenz indiziert. Zur analgetischen Therapie der biliären Kolik werden Spasmolytika in Kombination mit Analgetika eingesetzt. Schwächer wirksame Analgetika, z. B. Metamizol [503] oder Paracetamol, können ausreichen, oder aber es werden stärker analgetisch wirksame Opiatderivate wie Pethidin oder Buprenorphin, die den Sphincter Oddi relativ wenig kontrahieren [504 - 506] nötig. Außer mit den angegebenen Medikamenten sind Gallenkoliken aber auch mit Nitroglyzerin erfolgreich behandelt worden [507].

Es ist gezeigt worden, dass NSAR wie Diclofenac (z. B. $75 \mathrm{mg}$ i. m.) $[508,509]$ oder Indometacin (z. B. $50 \mathrm{mg}$ i.v. oder 2-mal 75 mg Supp.) [510, 511] bei der biliären Kolik eine gute analgetische Wirksamkeit haben. Randomisierte kontrollierte Studien zeigen, dass durch die Gabe von NSAR in der o. a. Dosis auch die Wahrscheinlichkeit, im Verlauf einer biliären Kolik eine akute Cholezystitis zu entwickeln, gesenkt werden kann [509, 512]. Ob die „traditionelle“ Behandlung der biliären Kolik oder die Therapie mit NSAR zu einer effizienteren Schmerzlinderung führen, kann gegenwärtig aufgrund fehlender vergleichender Studien nicht entschieden werden. Die Wertigkeit verschiedener Medikamentenkombinationen wurde bisher ebenfalls nicht ausreichend untersucht.

\section{EMPFEHLUNG IIIA.4.}

Bei akuter Cholezystitis mit Zeichen der Sepsis, Cholangitis, Abszess oder Perforation sollen unverzüglich Antibiotika verabreicht werden (A, II, starker Konsens, NKLM).

Zur Antibiotikatherapie bei unkomplizierter Cholezystitis liegt keine Evidenz vor (Statement, V, starker Konsens).

\section{Kommentar:}

Die Therapie der akuten Cholezystitis erfolgt in der Regel chirurgisch mittels Cholezystektomie. Es gibt wenige Daten zur medikamentösen Therapie der akuten Cholezystitis. Neuere Studien stellen bei der leichtgradigen akuten Cholezystitis (keine Organdysfunktion, milde lokale Entzündungszeichen, Fieber $\leq 39^{\circ} \mathrm{C}$, Leukozyten $\leq 18 \mathrm{G} / \mathrm{L}$ ) den Nutzen der Antibiotika in Frage, da sich Komplikationen und Krankenhausverweildauer in einer kleinen randomisierten Studie bei 84 Patienten nicht unterschieden 
[513]. In den Tokyo-Leitlinien werden in dieser Situation keine Antibiotika empfohlen, wenn zudem die ASA-Risikoklasse $\leq$ II ist und nur wenige Komorbiditäten vorliegen (Charlson Comorbidity Index $\leq 5)$ [514, 515]. Generell kann früh von intravenöser auf orale Gabe umgestellt werden [516]. Eine große randomisierte kontrollierte Studie hat nachgewiesen, dass bei Patienten mit akuter leicht- bis mittelgradiger Cholezystitis (d. h. ohne kritische Faktoren wie Schock, Bewusstseinsstörung oder Organversagen) [514] die Antibiose postoperativ nicht fortgeführt werden muss [517]. Die gleichzeitige Gabe von NSAR wirkt nicht nur schmerzlindernd, sondern könnte die Progression der Cholezystitis vermeiden, möglicherweise weil es sich initial häufig um eine abakterielle Entzündung handelt [509].

Die Paul-Ehrlich-Gesellschaft hat in ihren aktualisierten Empfehlungen zur kalkulierten Initialtherapie bei Erwachsenen mit sekundärer Entzündung der Gallengänge eine kurze 3- bis 5-tägige Therapie [518] mit Ampicillin+Sulbactam, Moxifloxacin und Kombinationstherapien von Fluorochinolonen der Gruppe 2/3 (Ciprofloxacin/Levofloxacin) bzw. Cephalosporinen der Gruppe 3a/4 (Ceftriaxon/Cefepim) mit Metronidazol empfohlen [519]. Piperacillin + Tazobactam oder Carbapeneme Gruppe 1/2 werden bei Vorliegen weiterer Risikofaktoren eingesetzt. Insgesamt basieren alle diese Empfehlungen im Wesentlichen auf Expertenmeinungen; größere kontrollierte vergleichende Studien fehlen. Üblicherweise liegt eine Mischflora mit der Dominanz von gramnegativen Erregern (E. coli, Enterobacter, Klebsiellen), aber auch grampositiven Erregern (Enterokokken, Staphylokokken) vor. Anaerobier werden bei Infektionen der Gallengänge in 5-10\% der Fälle nachgewiesen. Kontrollierte Studien, die für die empirische Therapie den Einsatz von Metronidazol z. B. in Kombination mit Cephalosporinen begründen, liegen nicht vor. Studien zeigen, dass z. B. die 30-Tage-Letalität bei durch ESBL-bildende Enterokokken verursachter Cholangitis deutlich über der Letalität von Patienten mit Nicht-ESBL-Bildnern, die von Carbapenem erfasst werden, liegt (14 vs. $3 \%$ ). Diese erhöhte Letalität ist insbesondere durch die Verzögerung in der Einleitung einer effektiven antibiotischen Therapie begründet. Bei Sepsis ist die Antibiotikatherapie ohne Verzögerung einzuleiten.

\section{IIIB. Chirurgische Therapie}

EMPFEHLUNG IIIB. 1.

Bei unkomplizierter Cholezystolithiasis mit charakteristischen biliären Schmerzen sollte eine Cholezystektomie erfolgen (B, I, starker Konsens, NKLM).

\section{Kommentar:}

1. Die Therapieziele der Cholezystektomie bestehen in:

2. Verhinderung oder Verminderung erneuter biliärer Schmerzen

3. Verhinderung späterer oder Beseitigung bestehender Komplikationen der Cholezystolithiasis

4. Prävention des Gallenblasenkarzinoms bei Patienten mit hohem Risiko (siehe IIIB.4 -6)
Patienten mit charakteristischen biliären Schmerzen und unkomplizierter Cholezystolithiasis haben abhängig von Intensität und Anzahl der symptomatischen Episoden ein erhöhtes Risiko neuerliche Koliken zu bekommen [520]. Das Risiko, Komplikationen zu entwickeln, beträgt 1 - $3 \%$ pro Jahr [132, 134, 135, 521]. Allgemeine Kontraindikationen gegen eine Operation oder ein dezidierter Patientenwunsch können dem entgegenstehen. Die randomisierte National Cooperative Gallstone Study [131] bot die Möglichkeit, den natürlichen Spontanverlauf bei Patienten zu beobachten, die keine Litholyse mit Chenodeoxycholsäure erhielten. Von diesen Patienten entwickelten 69\% innerhalb von zwei Jahren erneut biliäre Symptome, und $4 \%$ der Patienten mussten akut cholezystektomiert [131] werden.

Während die Cholezystektomie die Komplikationen der Gallenblasensteine verhindert, lässt sich das Komplikationsrisiko von in den Gallengang übergetretenen Steinen, insbesondere das der biliären Pankreatitis, auch durch die endoskopische Papillotomie (EPT) verhindern. Falls die Gallenblase in dieser Situation funktionstüchtig und steinfrei ist, muss nicht cholezystektomiert werden. Nach einer Cholezystektomie können abdominelle Beschwerden mit meist unspezifischem Charakter bei jedem dritten bis vierten Patienten persistieren [130, 522 - 528].

\section{EMPFEHLUNG IIIB.2.}

Die Routinegabe einer Antibiotikaprophylaxe ist bei der elektiven laparoskopischen Cholezystektomie bei „low-risk“-Patienten nicht notwendig (A, I, starker Konsens).

\section{Kommentar:}

Die elektive laparoskopische Cholezystektomie ist ein weit verbreiteter Eingriff. Die perioperative Antibiotikaprophylaxe wurde bisher kontrovers diskutiert, und ein Vorteil konnte bisher nicht eindeutig belegt werden.

Die niedrige Rate an Wundinfektionen und potenziellen systemischen Infektionen bei Patienten ohne wesentlich erhöhtes Risiko erklärt, dass kontrollierte Studien zur Klärung der Evidenz eines Vorteils oder nicht vorhandenen Vorteils einer hohen Anzahl von Patienten bedürfen [529 - 535]. Insofern werden Metaanalysen herangezogen [536-538]. Die meisten kontrollierten Studien liegen für Patienten mit niedrigem Risiko vor, bei denen wenige Komorbiditäten wie Diabetes mellitus oder Immunsuppression vorliegen, infektiöse Komplikationen und eine Konversion zur offenen Cholezystektomie nicht zu erwarten sind und das Narkoserisiko niedrig ist [537]. Die Metaanalysen sagen aus, dass eine perioperative Antibiotikaprophylaxe im Rahmen einer elektiven laparoskopischen Cholezystektomie bei diesen Patienten nicht notwendig ist [537, 538]. Ein Cochrane-Review schließt 11 randomisierte Studien mit „low-risk“-Patienten ein. Die Wundinfektionsrate war über alle Studien sehr ähnlich (24/900 bzw. 2,7\%; in der Prophylaxe-Gruppe; 25/764 bzw. 3,3\% in der Nicht-Prophylaxe-Gruppe). Die systemische Infektionsrate unterschied sich ebenfalls nicht. Die Autoren schließen, dass die Evidenz, eine Antibiotika-Prophylaxe abzulehnen oder zu empfehlen, nicht ausreicht [536]. Schwedische Registerdaten [539] bei 13911 Patien- 
ten zeigten in einer multivariaten Analyse keinen Nutzen einer Antibiotikaprophylaxe. Eine Antibiotikaprophylaxe war bei $68 \%$ des Gesamtkollektivs durchgeführt worden. Im Anschluss an die beiden Metaanalysen wurden zwei weitere randomisierte Studien veröffentlicht. Eine monozentrische Studie fand bei „low-risk“-Patienten keinen Effekt einer einmaligen Cefuroxim-Gabe (4,5\% Wundinfektionsrate) im Vergleich zur Kontrollgruppe (4,0\%) [540]. Die andere Studie bei über 1000 japanischen Patienten fand eine Gesamtinfektionsrate von $0,8 \%$ in der Antibiotikagruppe und $3,7 \%$ in der Kontrollgruppe $(P=0,001)$. Die Daten sind kritisch zu sehen, weil es sich nicht um eine Antibiotikaprophylaxe handelt (stattdessen 1-mal intraoperativ, 1-mal nach $12 \mathrm{~h}$ und 1-mal nach 24 h). Die Gesamtinfektionsrate von 3,7 \% in der Kontrollgruppe erscheint relativ hoch [541]. Registerdaten aus Bayern an über 25000 Cholezystektomien berichten lediglich Infektionsraten von 1,2-1,4\%. Insgesamt zeigt sich, dass bei der elektiven laparoskopischen Cholezystektomie lokale und systemische Infektionen selten sind. Nach den publizierten Daten ist die Antibiotikaprophylaxe daher nicht generell notwendig.

Die Paul-Ehrlich-Gesellschaft sieht allerdings eine Indikation zur Antibiotikaprophylaxe bei akuter Cholezystitis, Eingriffen am Ductus choledochus und Notfalloperationen [542]. Als weitere Risiken, bei denen eine Antibiotikaprophylaxe in Erwägung gezogen werden sollte, gelten die intraoperativ eröffnete Gallenblase, die Laparotomie bzw. offene Cholezystektomie sowie die Konversion vom laparoskopischen zum offenen Vorgehen. Als generelle Risikofaktoren für postoperative Wundinfektionen gelten die allgemeinen Risikofaktoren wie von Wacha et al. [542] beschrieben. Obwohl es für die Gruppe der sogenannten „high-risk“-Patienten bei laparoskopischer Cholezystektomie keine randomisiert kontrollierten Daten gibt, wird eine risikoadaptierte und individualisierte Antibiotikaprophylaxe durchgeführt [543]. Das Risiko beginnt mit dem Eingriff. Ein wirksamer Antibiotikaspiegel ist für die Dauer der Risikoperiode sicherzustellen. Eine perioperative Antibiotikagabe sollte im besten Fall eine Stunde vor bis zu zwei Stunden nach der geplanten Operation erfolgen, spätestens jedoch vor Wundverschluss. Hierfür sind die entsprechenden Antibiotika und Dosierungen zu berücksichtigen ( $\triangleright$ Tab. 2). Als geeignetes Antibiotikum gelten ein Aminopenicillin mit Betalactamasehemmer oder ein Cephalosporin der 1. und 2. Generation. Die Fortführung der perioperativen Prophylaxe über die Operation hinaus als präventive Therapie bedarf einer besonderen Indikation [543-546].

Der Effekt einer Antibiotikaprophylaxe muss abgewogen werden gegen mögliche Nebenwirkungen, Ausbreitung von Antibiotikaresistenzen und Kosten [535, 547].

\section{EMPFEHLUNG IIIB.3.}

Die asymptomatische Cholezystolithiasis ist in der Regel keine Indikation zur Cholezystektomie (Statement, III, starker Konsens, NKLM).
- Tab. 2 Dosierung von Antibiotika in der perioperativen Prophylaxe (aus: [542]).

\begin{tabular}{|l|l|}
\hline Antibiotikum & Dosierung (pro Tag) \\
\hline Ampicillin & $5 \mathrm{~g}$ \\
\hline Ampicillin/Sulbactam & $2 \mathrm{~g} / 1 \mathrm{~g}$ \\
\hline Amoxacillin/Clavulansäure & $2 \mathrm{~g} / 0,2 \mathrm{~g}$ \\
\hline Piperacillin & $4 \mathrm{~g}$ \\
\hline Piperacillin/Tazobactam & $4 \mathrm{~g} / 0,5 \mathrm{~g}$ \\
\hline Mezlocillin & $4 \mathrm{~g}$ \\
\hline Cefazolin & $2 \mathrm{~g}$ \\
\hline Cefuroxim & $1,5 \mathrm{~g}$ \\
\hline Cefotaxim & $2 \mathrm{~g}$ \\
\hline Ceftriaxon & $2 \mathrm{~g}$ \\
\hline Ceftazidim & $2 \mathrm{~g}$ \\
\hline Cefepim & $2 \mathrm{~g}$ \\
\hline Imipenem/Cilastatin & $0,5 \mathrm{~g} / 0,5 \mathrm{~g}$ \\
\hline Meropenem & $1 \mathrm{~g}$ \\
\hline Ertapenem & $1 \mathrm{~g}$ \\
\hline Clindamycin & $600 \mathrm{mg}$ \\
\hline Metronidazol & $500 \mathrm{mg}$ \\
\hline Gentamicin & $240 \mathrm{mg}(\mathrm{bis} \mathrm{zu} 5 \mathrm{mg} / \mathrm{kg}$ \\
\hline Doxycyclin & $200 \mathrm{mg}$ \\
\hline Cotrimoxazol & $960 \mathrm{mg}$ \\
\hline Vancomycin & $1 \mathrm{~g}$ \\
\hline Teicoplanon & $400 \mathrm{mg}$ \\
\hline Ciprofloxacin & $400 \mathrm{mg}$ \\
\hline Levofloxacin & $500 \mathrm{mg}$ \\
\hline
\end{tabular}

\section{Kommentar:}

Weder umfangreiche klinische Beobachtungen noch detaillierte Analysen prospektiver Studien zum klinischen Verlauf der asymptomatischen Cholezystolithiasis belegen den Nutzen der Cholezystektomie bei asymptomatischen Steinträgern. Zum natürlichen Verlauf der asymptomatischen Gallensteinerkrankung gibt es acht Studien, in denen insgesamt 1403 Patienten über einen Zeitraum von 2-25 Jahren beobachtet wurden. In diesem Zeitraum blieben 60-80\% der Patienten asymptomatisch. Die Wahrscheinlichkeit, biliäre Symptome zu entwickeln, beträgt in den ersten 5 Jahren 2-4\% pro Jahr und halbiert sich in den folgenden fünf Jahren auf 1 - 2 \% pro Jahr [132, $134-138]$. Die jährliche Inzidenz von Komplikationen liegt bei 0,1-0,3\%, wobei den meisten Komplikationen biliäre Koliken vorausgehen [132, 134 -136]. Für die offene Cholezystektomie wurde nachgewiesen, dass die Operation bei asymptomatischen Gallensteinträgern deren Lebenserwartung nicht erhöht [133, 548]. Darüber hinaus sind die Kosten geringer, wenn man sich bei asymptomatischen Gallensteinträgern abwartend verhält und keine prophylaktische Cholezystektomie durchführt. In Ländern mit niedriger Gallenbla- 
senkarzinom-Prävalenz [549] rechtfertigt das leicht erhöhte, aber immer noch sehr niedrige Gallenblasenkarzinom-Risiko bei der asymptomatischen Cholezystolithiasis nicht den Eingriff [550, 551]. Auch Diabetiker bedürfen keiner prophylaktischen Therapie $[552,553]$.

Hinweis: Diese Leitlinien-Empfehlung wurde für die „Klug entscheiden “-Initiative der Deutschen Gesellschaft für Innere Medizin ausgewählt [554].

\section{EMPFEHLUNG IIIB.4.}

Asymptomatische Patienten mit einer Porzellangallenblase sollten cholezystektomiert werden (B, III, starker Konsens).

\section{Kommentar:}

Die Porzellangallenblase wird in der Literatur immer noch als Risikofaktor für die Entstehung des Gallenblasenkarzinoms beschrieben. Gemäß der Literatur wird die Entartungsrate bei einer Porzellangallenblase bis $62 \%$ angegeben, wenngleich aktuelle Serien geringere Raten angeben. Auch die stippchenartige, multiple partielle Kalzifizierung der Gallenblasenwand ist mit einem erhöhten Karzinomrisiko verbunden. Hier ist eine prophylaktische Cholezystektomie weiterhin unverändert anzuraten. Neuere Studien bezüglich Porzellangallenblasen aus westlichen Ländern liefern niedrigere Entartungsraten, aber können auch nur mit sehr kleinen Fallzahlen dienen, sodass die Prozentangaben hier nicht als repräsentativ angesehen werden können [555559].

\section{EMPFEHLUNG IIIB.5.}

Bei asymptomatischen Patienten mit Gallenblasensteinen $>3 \mathrm{~cm}$ Durchmesser sollte eine Cholezystektomie erwogen werden (B, II, starker Konsens).

\section{Kommentar:}

Gallensteine ab einer Größe $>3 \mathrm{~cm}$ werden in der Literatur immer noch als Risikofaktoren in der Entstehung des Gallenblasenkarzinoms beschrieben. Gemäß der Literatur verzehnfacht sich das Risiko bei Steinen $>3 \mathrm{~cm}$. Cariati et al. [557] haben sich umfassend mit der Histologie und der Entartung des Gallenblasenkarzinoms befasst und beschreiben den Stein $>3 \mathrm{~cm}$ als Risikofaktor. Gerade bei adenosquamösen und squamösen (Plattenepithel-) Karzinomen sind lange bestehende Cholesterinsteine oder gemischte Steine bereits ab 1,5 cm ein Risikofaktor [557, 558, 560].

\section{EMPFEHLUNG IIIB.6.}

Patienten mit Cholezystolithiasis und Gallenblasenpolypen $\geq 1 \mathrm{~cm}$ sollten unabhängig von der Symptomatik cholezystektomiert werden (B, II, starker Konsens).

\section{Kommentar:}

Die Prävalenz von Gallenblasenpolypen liegt zwischen 1 und $7 \%$ [561 - 564], in Deutschland beträgt sie bis zu $6 \%[565,566]$. Die Adenom-Prävalenz liegt unter $5 \%$ [563, 566]. In mehreren großen Studien [557, 563, 564, 567 - 570] hatten solitäre Polypen $\geq 1 \mathrm{~cm}$ Durchmesser eine deutlich erhöhte Wahrscheinlichkeit, neoplastischer Genese (Adenome) zu sein, und in bis zu $50 \%$ das Risiko, ein Karzinom zu tragen [563, 564, 567 - 571], sodass diese Patienten cholezystektomiert werden sollten. Bei Polypen $<1 \mathrm{~cm}$ Durchmesser war das Karzinomrisiko deutlich geringer [572 - 576], eine Polypengröße $\leq 4,15 \mathrm{~mm}$ schloss in einem systematischen Review Malignität aus [576]. Falls die Patienten jedoch gleichzeitig biliäre Symptome oder weitere Risikofaktoren für eine Adenomentwicklung (Alter > 50 Jahre, Größenzunahme des Polypen, Gallensteine, fokale Gallenblasenwandverdickung) haben, kann auch in diesen Fällen eine Cholezystektomie durchgeführt werden [563, 569, 574 - 581]. Bei Polypen > 18-20 mm kann wegen des signifikanten Malignitätsrisikos primär eine offene Cholezystektomie geplant werden [570, 577, 582 - 584]. In Kliniken mit entsprechender Expertise kann bei Polypen > 18-20 mm die Cholezystektomie auch komplett laparoskopisch durchgeführt werden [585, 586].

Bei Polypen $<1 \mathrm{~cm}$, die nicht operiert werden, werden sonografische Verlaufskontrollen empfohlen: zunächst nach 6 Monaten, dann jährlich, wenn keine Größenzunahme vorliegt, über 5 Jahre [564, 570, 581]. Während der Sonografie sind Gallenblasenpolypen durch Umlagern des Patienten von Gallenblasensteinen zu differenzieren. Das Vorliegen von mehr als einem Polypen spricht gegen ein Adenom und für das Vorliegen von Cholesterolpolypen. Wenn der Polyp im Follow-up nicht mehr nachweisbar ist, handelte es sich wahrscheinlich um einen Pseudopolypen und weitere Kontrollen sind nicht indiziert [581]. Gallenblasenpolypen können mit der Endosonografie präziser als mit der transkutanen Sonografie erfasst werden ( $80-97 \%$ vs. $52-76 \%$ ) [587 - 589]. Bei Gallenblasenpolypen $>5 \mathrm{~mm}$, die in der transkutanen Sonografie suspekt sind, kann die Endosonografie zur Abgrenzung von einem Gallenblasenkarzinom hilfreich sein [179, 590, 591].

Die Situation von polypoiden Gallenblasenveränderungen ohne gleichzeitige Cholezystolithiasis wurde im Konsensus nicht berücksichtigt.

\section{EMPFEHLUNG IIIB.7.}

Im Rahmen onkologisch resezierender Eingriffe am Magen und Ösophagus mit systematischer Lymphadenektomie sollte eine simultane Cholezystektomie durchgeführt werden (B, I, starker Konsens).

Die Cholezystektomie im Rahmen der Adipositaschirurgie sollte ausschließlich bei symptomatischen Steinträgern erfolgen (B, II, starker Konsens).

Im Rahmen größerer malresorptiver Eingriffe am Dünndarm kann eine simultane Cholezystektomie bei asymptomatischen Patienten erfolgen (0, III, starker Konsens). 


\section{Kommentar:}

Das Auftreten von Gallensteinen und Sludge ist bei Patienten nach Gastrektomie höher als in der Gesamtbevölkerung [592]. Diese höhere Inzidenz ist wahrscheinlich auf die chirurgische Präparation des N. vagus und dessen Ramus hepaticus [593] sowie die durchgeführte Magen-Darm-Rekonstruktion zurückzuführen [594]. Daher präferieren einige Chirurgen routinemäßig die simultane Cholezystektomie.

Das Auftreten von Gallensteinen nach resezierenden Eingriffen am Magen oder Ösophagus wurde im Rahmen einer Metaanalyse aus 16 Studien mit 17,5\% beziffert, was 2- bis 4-fach höher als in einem nach Alter und Geschlecht gematchten Vergleichskollektiv war. Die weitaus größte Zahl der eingeschlossenen Studien hat jedoch retrospektiven Charakter. Die Operationsverfahren unterscheiden sich stark. Bei 12 der eingeschlossenen Studien wurden Angaben zur Ausbildung einer symptomatischen Cholezystolithiasis im Verlauf gemacht. Bei 4,7\% der operierten Patienten war im Verlauf eine Cholezystektomie erforderlich. Da die zusätzliche Morbidität $(0,95 \%)$ einer simultan durchgeführten Cholezystektomie höher war als die der nachfolgenden Cholezystektomie $(0,45 \%)$ - die nach den Angaben der Autoren in der Mehrheit auch laparoskopisch durchgeführt werden kann - besteht laut den Autoren keine Notwendigkeit zur simultanen Cholezystektomie.

Demgegenüber steht aber die Tatsache, dass die Steinbildung nach onkologischer Gastrektomie mit dem Ausmaß der Lymphadenektomie steigt. Daten aus einer monozentrisch retrospektiven Studie zeigen, dass im Mittel 25\% aller Patienten nach onkologischer Gastrektomie im Verlauf Gallensteine entwickeln. Im Rahmen der erweiterten Lymphadenektomie lagen die Daten über $40 \%$.

Weiterhin belegen Daten aus einer japanischen Registerstudie ( $n=14006$ ), dass im Rahmen einer laparoskopischen onkologischen Magenresektion weder die Laparoskopie-assoziierten Komplikationen noch die Dauer des stationären Aufenthaltes durch eine zusätzlich durchgeführte Cholezystektomie $(n=1484)$ anstiegen.

Die CHOLEGAS-Studie [595] ist die einzige Studie, die multizentrisch und prospektiv randomisiert den Effekt einer simultanen Cholezystektomie im Rahmen der onkologischen Gastrektomie untersucht. Nach Einschluss von 65 Patienten in jedem Studienarm zeigen die vorliegenden Daten [596], dass die simultane Cholezystektomie die hepatobiliären Komplikationen nicht erhöht und dass die postoperative Letalität gleich ist. Ebenso vergleichbar waren die Operationsdauer, der geschätzte intraoperative Blutverlust sowie die Dauer des postoperativen stationären Aufenthaltes. Bei einer nur geringen Rekrutierung von 5 Patienten je Studienzentrum $(n=9)$ können Fragen nach der Inzidenz von Gallensteinen nach onkologischer Gastrektomie sowie deren chirurgische Therapiebedürftigkeit bei einem geplanten Nachbeobachtungszeitraum von 5 Jahren zum gegenwärtigen Zeitpunkt noch nicht beantwortet werden.

Im Rahmen der metabolischen Chirurgie ist die Rate der simultan durchgeführten Cholezystektomien in den letzten Jahren deutlich zurückgegangen. Gründe hierfür sind die höhere Gesamtmorbidität und -letalität, die höhere Wiederaufnahmerate sowie eine höhere Re-Interventionsrate [597]. Im Vergleich der verschiedenen Verfahren ist das Risiko zur postoperativen Gallensteinbildung nach der laparoskopischen Roux-en-Y-Rekonstruktion im Vergleich zum Gastric banding und zur Sleeve resection am höchsten [598]. In einer Registerstudie stellen die Autoren fest, dass die simultane Cholezystektomie nur Patienten mit symptomatischen Gallensteinleiden vorbehalten sein sollte [597]. Dies wird weiterhin durch die Daten einer Metaanalyse untermauert [599]. Das Risiko für eine sekundäre Cholezystektomie nach vorangegangener laparoskopischer Roux-en-Y-Rekonstruktion betrug 6,8\% insgesamt. Hiervon fielen 5,3\% der symptomatischen Cholezystolithiasis zu. Die sekundäre Cholezystektomie war in den meisten Fällen (95,5\%) auch laparoskopisch mit einer geringen Morbidität (1,8\%) durchführbar, sodass als Fazit die Gelegenheit-Cholezystektomie bei fehlendem Steinnachweis nicht empfohlen wird und ausschließlich symptomatischen Steinträgern vorbehalten sein soll.

Das Risiko zur Ausbildung von Gallensteinen ist generell beim Vorliegen eines M. Crohn im Vergleich zur Normalbevölkerung nahezu verdoppelt. Weitere unabhängige Risikofaktoren sind ein ileokolischer Befall, eine Krankheitsdauer von mehr als 15 Jahren, mehr als drei Rezidive sowie mehr als $30 \mathrm{~cm}$ Dünndarmverlust oder ein Kurzdarmsyndrom mit weniger als $120 \mathrm{~cm}$ verbliebenem Dünndarm [600 - 602]. Generell scheint die Operationsbedürftigkeit von Gallensteinen durch einen vorliegenden M. Crohn im Vergleich zur Normalbevölkerung nicht erhöht zu sein [601], jedoch ist ein M. Crohn als unabhängiger Risikofaktor für postoperative Komplikationen bei primären Cholezystektomien zu werten [603]. Prospektiv randomisierte Daten zu malresorptiven Operationen am Kolon und Dünndarm fehlen bis dato gänzlich. Für die Cholezystektomie sprechen ein zu erwartend langer Krankheitsverlauf mit langem Überleben sowie ein zu erwartendes Kurzdarmsyndrom [600].

\section{EMPFEHLUNG IIIB.8.}

Die akute Cholezystitis ist eine Indikation zur frühzeitigen laparoskopischen Cholezystektomie (A, I, starker Konsens, NKLM).

Diese sollte innerhalb von $24 \mathrm{~h}$ nach stationärer Aufnahme erfolgen ( $\mathrm{B}, \mathrm{I}$, starker Konsens).

\section{Kommentar:}

Die akute Cholezystitis ist die häufigste Komplikation des Gallensteinleidens. Bei $90 \%$ der Patienten mit akuter Cholezystitis ist die Ursache ein passagerer oder dauerhafter Verschluss des Ductus cysticus durch einen Gallenstein. In der Regel gilt hier die laparoskopische Cholezystektomie als Standard.

Eine der wesentlichen Fragen bei der Behandlung der akuten Cholezystitis bestand in der Vergangenheit darin, den optimalen Zeitpunkt für die laparoskopische Cholezystektomie zu finden. Es war unklar, ob zunächst eine antibiotische Vorbehandlung des Patienten und die Operation im symptomfreien Intervall erfolgen sollten, oder ob eine frühe Operation oder nicht sogar die Operation innerhalb von $24 \mathrm{~h}$ der beste Weg für den Patienten sei. Allein die Definition „frühe Operation“ wurde in der Literatur höchst un- 
terschiedlich definiert. So wurde in einigen Studien die frühe Operation innerhalb von 7 Tagen nach Symptombeginn, in anderen Studien innerhalb von $72 \mathrm{~h}$ nach Symptombeginn, definiert. Auch bestand Uneinigkeit darüber, ob der Beginn des Zeitintervalls mit Beginn der Symptome oder der Vorstellung bzw. der Aufnahme des Patienten in einer Klinik festgelegt wird [604-611].

Die neuesten Untersuchungen sehen klare Vorteile für eine unverzügliche chirurgische Intervention, einer sogenannten „immediate cholecystectomy“. Dies bedeutet, dass der Patient mit der Diagnose Akute Cholezystitis innerhalb von $24 \mathrm{~h}$ nach Aufnahme laparoskopisch cholezystektomiert werden soll. Diese klinische Diagnose soll zügig und eindeutig gestellt werden. Sie basiert auf 3 der 4 folgenden Symptome: Rechtsseitige Oberbauchschmerzen, Murphy's-Zeichen, Leukozytose und Fieber. Zusätzlich sollen eine Cholezystolithiasis (Konkremente oder Sludge) oder sonografische Zeichen der Cholezystitis (verdickte Gallenblasenwand) vorliegen. Unter Anwendung dieser Kriterien kann die Diagnose „Akute Cholezystitis“ eindeutig und nachvollziehbar gestellt werden. Steht die Diagnose fest und liegt eine Operationsfähigkeit des Patienten vor, soll die laparoskopische Cholezystektomie innerhalb von $24 \mathrm{~h}$ nach Aufnahme angestrebt werden, unabhängig davon, wann erstmals Symptome aufgetreten sind $[612,613]$.

In einer Registerstudie wurden insgesamt 4113 Patienten analysiert, die aufgrund einer akuten Cholezystitis zwischen 1995 und 2006 einer laparoskopischen Cholezystektomie unterzogen wurden. Die Untersuchung basiert auf dem Register der Swiss Association of Laparoscopic and Thoracoscopic Surgery. Dabei wurden die Patienten unterschiedlichen Operationszeitpunkten zugeordnet. Es zeigte sich, dass die Patienten, die innerhalb von $24 \mathrm{~h}$ nach Aufnahme operiert wurden, nur halb so viel postoperative Komplikationen entwickelten, wie die Patienten, die erst $>6$ Tage nach Aufnahme operiert wurden (6 vs. $13 \%$ ). Ebenso stieg die Konversionsrate von 12 auf $28 \%$ und die Re-Operationsrate von 0,9 auf 3,0\% bei der späteren Operation [614].

Die derzeit weltweit größte randomisierte Untersuchung zur akuten Cholezystitis ist die ACDC-Studie. Es handelt sich um eine interdisziplinäre, multizentrische, prospektiv-randomisierte Studie, die gemeinsam von Gastroenterologen und Chirurgen durchgeführt wurde. Es zeigen sich klare Vorteile für eine unverzügliche laparoskopische Cholezystektomie innerhalb von $24 \mathrm{~h}$ nach stationärer Aufnahme der Patienten mit akuter Cholezystitis. Dabei wurden 304 Patienten in eine frühe Gruppe (Cholezystektomie innerhalb von $24 \mathrm{~h}$ ) und 314 in eine späte Gruppe (Cholezystektomie zwischen Tag 7 und 45 nach Aufnahme) eingeschlossen. Bezüglich des primären Endpunktes, Morbidität am Tag 75, gibt es ein eindeutiges Ergebnis zugunsten der frühen Gruppe: 12 vs. $33 \%$. Die Hospitalisierungsdauer ist in der frühen Gruppe halb so lang, die Gesamtkosten des Klinikaufenthaltes unterscheiden sich ebenfalls signifikant zugunsten der frühen Gruppe. Dagegen gibt es keinen signifikanten Unterschied zwischen den Gruppen bezüglich der Konversionsrate zur offenen Operation (10 vs. 12\%) [167].

Eine weitere randomisierte Studie zur akuten Cholezystitis, bei der entweder frühzeitig $72 \mathrm{~h}$ nach Symptombeginn oder später (frühestens nach 6 Wochen) operiert wurde, bestätigte die geringere Morbidität der frühen Cholezystektomie in der deutlich grö-
- Tab. 3 ASA-Klassifikation.

ASA I normaler, gesunder Patient

ASA II Patient mit leichten Allgemeinerkrankungen

ASA III Patient mit schweren Allgemeinerkrankungen und Leistungsminderung

ASA IV Patient mit einer inaktivierenden Allgemeinerkrankung, die eine ständige Lebensbedrohung darstellt

ASA V moribunder Patient, von dem nicht erwartet wird, dass er die nächsten $24 \mathrm{~h}$ überlebt, sei es mit oder ohne Intervention

Beren ACDC-Studie [615], wobei die frühe Cholezystektomie Notfalleingriffe, die sonst bei $23 \%$ der spät operierten Patienten notwendig wurden, vollständig verhinderte. Der maximale Zeitraum bis zur frühelektiven Cholezystektomie nach Diagnosestellung bleibt damit noch ungeklärt, wobei sich $72 \mathrm{~h}$ nach Symptombeginn und $24 \mathrm{~h}$ nach Aufnahme gegenseitig nicht ausschließen.

Bei Patienten mit Begleiterkrankungen (z. B. akute Pankreatitis, ASA-Risikoklasse > III; $>$ Tab.3), bei denen eine unmittelbare laparoskopische Cholezystektomie nicht sinnvoll erscheint, wird der Operationszeitpunkt individuell festgelegt. Alternativ bzw. zeitüberbrückend kann bei Patienten mit akuter Cholezystitis und hohem Operationsrisiko eine perkutane oder endosonografisch gestützte Drainage der Gallenblase (Cholezystostomie) erfolgen (siehe IIIC.13). Die rein konservative Therapie der akuten Cholezystitis ist zwar möglich, allerdings kommt es nach alleiniger konservativer Behandlung bei über einem Drittel dieser Patienten zu Komplikationen oder Notaufnahmen wegen biliärer Schmerzen, und bei $30 \%$ wird im weiteren Verlauf eine Cholezystektomie erforderlich. Bei 10 bis $30 \%$ der Patienten mit akuter Cholezystitis treten schwere Komplikationen wie Gallenblasengangrän, -empyem oder -perforation auf. In diesen Situationen kann ein präoperatives CT hilfreiche Informationen liefern. Fisteln zwischen der Gallenblase und dem Gastrointestinaltrakt entwickeln sich bei weniger als $1 \%$ aller Gallensteinpatienten. Klinisch kann sich eine biliodigestive Fistel im späteren Spontanverlauf durch aszendierende Cholangitis oder Gallensäureverlustsyndrom manifestieren. In der Regel (60\%) handelt es sich um cholezystoduodenale Fisteln, die oft asymptomatisch sind. Wenn größere Steine durch die Fisteln abgehen, kann es zum Bild des Gallensteinileus kommen, insbesondere im terminalen Ileum. Der Nachweis der Aerobilie ist diagnostisch wegweisend für eine biliodigestive Fistel; zur weiteren Diagnostik sind Magnetresonanztomografie mit Magnetresonanzcholangiografie (MRCP) und endoskopische Verfahren geeignet [616-618].

\section{EMPFEHLUNG IIIB.9.}

Wird postoperativ ein Carcinoma in situ (Tis) oder ein Mukosakarzinom (T1a) festgestellt, ist die Entfernung der Gallenblase ausreichend (A, II, starker Konsens). 


\section{Kommentar:}

Gemäß der Daten des deutschen Zentralregisters „Okkultes Gallenblasenkarzinom “ ist eine einfache Cholezystektomie in Stadien kleiner als T1b, also speziell T1a oder PTis, ausreichend. Auch die Autoren, die eine radikale Operation ab T1b empfehlen, sehen grundsätzlich keine Indikation für ein radikales Vorgehen bei Karzinomen mit niedrigeren T-Stadien als T1b. In der Literatur gibt es somit einen Konsens, dass Gallenblasenkarzinome < T1b nicht radikal zu resezieren sind.

Es werden auch keine Lymphknotenmetastasen oder lymphatische oder perineurale Infiltration bei T1a-Tumoren beschrieben. T1a-Karzinome sind somit Tumoren, die üblicherweise weder einer Leberresektion noch einer Lymphadenektomie bedürfen [619-626].

\section{EMPFEHLUNG IIIB. 10.}

Falls nach Cholezystektomie wegen Cholezystolithiasis postoperativ ein Gallenblasenkarzinom im Stadium $\geq$ T1b nachgewiesen wird, soll bei kurativem Ansatz eine onkologische Nachresektion durchgeführt werden (A, III, starker Konsens).

\section{Kommentar:}

Falls nach Cholezystektomie wegen Cholezystolithiasis postoperativ ein Tumor im Stadium $\geq$ T1b nachgewiesen wird, ist grundsätzlich eine Reoperation mit kurativem Ansatz indiziert. Dies ist aufgrund der Daten- und Literaturlage zu empfehlen und stellt eine Ausweitung der bisherigen Leitlinienempfehlung auf das T-Stadium T1b dar. Da diese Empfehlung Auswirkungen auf die Überlebenswahrscheinlichkeit der Patienten mit Gallenblasenkarzinom haben kann, wurde trotz Evidenzgrad III von der Konsensuskonferenz der Empfehlungsgrad A festgelegt.

Die signifikante Prognoseverbesserung der Patienten im Tumorstadium T1b nach radikaler Leberresektion und Lymphadenektomie wurde mehrfach $[622,623]$ und auch an einem Kollektiv von 883 Patienten mit 109 T1b-Karzinomen im deutschen Zentralregister [626] dokumentiert. Die Leitlinien des National Comprehensive Cancer Network (NCCN) in den USA [627] und der European Society for Medical Oncology [628] empfehlen ebenfalls eine solche radikale Resektion bereits ab dem T1b-Gallenblasenkarzinom. Auch das französische Register (AFC-GBC2009 Study Group) mit insgesamt 218 inzidentellen Gallenblasenkarzinomen zeigt eine Verbesserung für die reoperierten T1b-Patienten [629, 630]. In einer Studie von D’Angelica et al. [631] wird die radikale Cholezystektomie (Leberresektion + Lymphadenektomie) nach Analyse der Fälle von 1988 bis 2002 des Memorial Sloan-Kettering Cancer Center ab einem Tumorstadium T1b empfohlen. Studien von Yi et al. [632] oder D’Hondt et al. [633] zeigten eine Verbesserung des medianen Überlebens für reoperierte T1b-Karzinome. Cavallaro et al. [625] geben eine Rezidivrate von bis zu 50 \% bei Unterlassung einer RR im Stadium T1b an. Hari et al. [634] kommen nach Analyse des Surveillance, Epidemiology and End Results Program (SEER) des National Cancer Institute in den USA mit der größten Studie im Stadium I (AJCC-Stadien T1a und T1b) mit 2788 Patienten im Stadium I zum Schluss, dass die radikale Resektion von T1b-Karzinomen zu einem Prognosevorteil führt. Von Hari et al. [634] sowie Andren-Sandberg und Deng [635] wird auf das Problem eines inkompletten Stagings bei Unterlassung einer radikalen Cholezystektomie hingewiesen.

Die Wedge-Resektion von $2-3 \mathrm{~cm}$ im Gallenblasenbett scheint bezogen auf die Radikalität des Resektionsumfangs ausreichend, sofern hiermit die R0-Situation zu erreichen ist. Bezogen auf die Leberresektionstechniken gibt es allerdings in der Literatur keinen Konsensus bezüglich des Ausmaßes eines angemessenen Resektionsumfanges für die verschiedenen T-Stadien. D’Angelica et al. [631] empfehlen eine Segment-IVb/V-Resektion ab einem T1bKarzinom; hier wurde aber die Wedge-Resektion nicht in die Evaluation miteinbezogen, sondern nur die Segment-IVb/V-Resektion gegenüber radikaleren Techniken verglichen. Jayaraman et al. [636] empfehlen für T1b-Karzinome eine Wedge- oder Segment-IVb/V-Resektion, für T2-Karzinome eine Segment-IVb/ V-Resektion und bei T3-Karzinomen eine Hemihepatektomie rechts, stets kombiniert mit einer Lymphadenektomie. Die Zentralregisterdaten zeigen tendenziell für die Wedge-Resektionstechnik die besten 5-Jahres-Überlebensraten bei T1b-Karzinomen; die Segment-IVb/V-Resektionen schneiden schlechter ab [626]. Bei T2-Tumoren zeigen die Daten des Zentralregisters tendenziell für die Segment-IVb/V-Resektion geringfügig bessere 5-Jahres-Überlebensraten verglichen mit der Wedge-Resektionstechnik, andere Techniken spielen keine Rolle. Dies ist auch deswegen relevant, da die Leitlinie bei T2/T3-Tumoren in weniger als $50 \%$ der Kliniken umgesetzt wird und Kliniken, die seltener Leberresektionen durchführen, auch seltener die Leitlinie in die Praxis umsetzen als „High volume“-Kliniken [637]. Nach mehr als 5 Jahren gleichen sich die Daten der Wedge-Resektion und Bisegmentektomie an. Bei den T3-Karzinomen hingegen sollte aufgrund der Daten des Zentralregisters mindestens eine Segment-IVb/V-Resektion durchgeführt werden [626, 638]. Die Kohortenstudie von Ethun et al. [639] bei 449 Patienten mit Gallenblasenkarzinomen - davon 120 Patienten mit T3 / T4-Karzinomen - weist darauf hin, dass das optimale Zeitintervall für die Nachresektion in dieser Gruppe bei 4 bis 8 Wochen nach der Cholezystektomie liegen könnte.

Die Angaben, bezogen auf das erforderliche Ausmaß der Leberresektion, sind in der Literatur uneinheitlich. Kohya et al. [640] beschreiben die Bisegmentektomie gegenüber der WedgeResektion bei T2-Karzinomen als das prognostisch bessere Verfahren. Araida et al. [641] konnten keine Unterschiede für T2- bis T3-Tumoren zwischen der Bisegmentektomie, Wedge-Resektion und Hepatektomie dokumentieren. Hierbei handelte es sich um eine Multicenterstudie mit 293 pT2- und 192 pT3-Karzinomen im Rahmen einer Umfrage der Japanese Society of Biliary Surgery. Es wird hier die Wedge-Resektion empfohlen, sofern hierdurch Tumorfreiheit (negative Resektionsränder) erreicht werden kann. Horiguchi et al. [642] haben nach Auswertung der Daten des Japan Biliary Cancer Registry von insgesamt 2067 Gallenblasenkarzinomen, darunter 415 T2-Karzinome und hiervon 161 Wedge-Resektion vs. 54 Segment-IVb/V-Resektionen, keine besseren Ergebnisse für die Segment-IVb/V-Resektion, jedoch eine höhere Komplikationsrate feststellen können. Auch Pawlik et al. [643] zeigen vergleichbare Ergebnisse für Patienten mit Hepatektomie, Bisegmentektomie und Wedge-Resektion. Die 5-Jahres- 
Überlebensraten bei T2-Tumoren im französischen Register (AFC-GBC-2009 Study Group) nach Wedge-Resektion betrugen $62 \%$, nach Segment-IVb/V-Resektion waren sie 59\% [629].

EMPFEHLUNG IIIB.11.

Die laparoskopische Cholezystektomie kann in jedem Trimenon einer Schwangerschaft bei dringlicher Indikation durchgeführt werden. Patientinnen, die bereits im 1. Trimenon symptomatisch geworden sind, sollten wegen erheblicher Rezidivgefahr im weiteren Verlauf ihrer Schwangerschaft früh elektiv operiert werden (B, III, starker Konsens).

\section{Kommentar:}

Gallenblasen-Sludge oder Gallensteine bilden sich bei mindestens $5 \%$ der Schwangeren aus; die Inzidenz gallensteinassoziierter Komplikationen während der Schwangerschaft liegt unter $1 \%$ [644]. Biliären Symptomen im ersten oder zweiten Trimenon folgt bei mehr als der Hälfte der betroffenen Schwangeren ein Beschwerderezidiv im weiteren Verlauf der Schwangerschaft [645 - 647]. Asymptomatische Steinträgerinnen werden nicht behandelt.

Die medikamentöse Therapie der symptomatischen Cholelithiasis in der Schwangerschaft basiert auf denselben Therapieprinzipien wie bei Nicht-Schwangeren. Zur Prüfung der Verträglichkeit und Gefahren von Medikamenten wird die Datenbank Embryotox (htttp://www.embryotox.de/) empfohlen. Nichtsteroidale Antiphlogistika (NSAID) sollten nur bis zur 28. SSW eingenommen werden; Fluorchinolone (und Imipenem) sollten wegen potenzieller Toxizität vermieden werden, während Penicilline ( \pm BetalactamInhibitor) und Cephalosporine in der Schwangerschaft sicher sind. Die konservative Behandlung der symptomatischen Cholezystolithiasis führt bei 38-69\% der Patientinnen zu rezidivierenden Symptomen und erhöht das Risiko für Komplikationen sowie stationäre Krankenhausaufenthalte [648-650]. Die Schwangerschaft ist zu keiner Phase eine Kontraindikation für eine laparoskopische Cholezystektomie [651 - 654]. Das frühelektive operative Vorgehen zur Verhinderung der hohen Rezidivwahrscheinlichkeit kann bei symptomatischer Cholezystolithiasis empfohlen werden, wohingegen ein zögerliches Vorgehen biliäre Komplikationen nach sich ziehen und das Kind gefährden kann. Insgesamt konnte aber kein sicherer prognostischer Unterschied hinsichtlich Frühgeburtlichkeit und fetaler Mortalität zwischen konservativem und chirurgischem Vorgehen nachgewiesen werden [655]. Zu beachten sind bei der laparoskopischen Cholezystektomie ein intraabdomineller Druck von maximal $15 \mathrm{mmHg}$ und ein intraoperatives $\mathrm{CO}_{2}$-Monitoring [656].

Ein konservatives Vorgehen bei symptomatischer Cholezystolithiasis während der Schwangerschaft führt zu einer über $50 \%$ igen symptombedingten Wiederaufnahmerate der Patientinnen innerhalb der ersten sechs Monate post partum [650, 657]. Patientinnen mit gleichzeitig vorliegenden Gallenblasen- und Gallengangsteinen, die nach der Gallengangsanierung asymptomatisch sind, können post partum (vorzugsweise ab der 6. Woche) cholezystektomiert werden [657].

\section{EMPFEHLUNG IIIB.12.}

Die laparoskopische Cholezystektomie sollte in einer 4-Trokartechnik durchgeführt werden (B, II, starker Konsens).

\section{Kommentar:}

Für die laparoskopische Cholezystektomie ist die 4-Trokartechnik als Standardtechnik zur Darstellung und Präparation der Strukturen des Calot'schen Dreiecks etabliert [658]. Operationstechniken mit einer reduzierten Anzahl an Zugängen können derzeit nicht als Standardtechniken empfohlen werden, sind jedoch bei ausreichender Expertise in erfahrenen Zentren möglich.

Große randomisierte Studien, die einen eindeutigen Vorteil für die Single-Incision-Techniken (SILS) oder Natural Orifice Transluminal Endoscopic Surgery (NOTES) zeigen, liegen nicht vor. Es zeigt sich vielmehr, dass die Ergebnisse sehr stark von der Erfahrung des Operateurs abhängig sind. Sowohl die Operationszeit als auch die Komplikationsrate dieser Verfahren zeigen eine starke Schwankungsbreite [659-663].

Besonders im Bereich der sogenannten Lernkurve können erhebliche Komplikationen auftreten [663, 664]. Der postoperative Schmerz wird weder durch NOTES noch Single-Incision-Techniken klinisch relevant verringert [661, 665-667].

Ein unter Umständen besseres kosmetisches Ergebnis wird als wesentlicher potenzieller Vorteil der SILS- und NOTES-Techniken gesehen [659-661, 663, 665].

\section{EMPFEHLUNG IIIB.13.}

Die postoperative Schmerztherapie nach laparoskopischer Cholezystektomie soll nach dem dreistufigen WHO-Stufenschema erfolgen (A, I, starker Konsens, NKLM).

\section{Kommentar:}

Hinsichtlich postoperativer Schmerzen nach laparoskopischer Cholezystektomie zeigt eine Metaanalyse aus 25 kontrolliert randomisierten Studien [668] keinen Vorteil zugunsten einer bestimmten Wirkstoffklasse (NSAR, Opioidanaloga, Antikonvulsiva). Alle Wirkstoffklassen reduzieren sowohl früh- als auch spät-postoperativ die Schmerzintensität auf der visuellen Analogskala und werden somit gleichermaßen empfohlen. Die Definition der jeweiligen Studienendpunkte und die Kombination der untersuchten Wirkstoffklassen ist uneinheitlich, sodass die Empfehlung einer speziellen Wirkstoffklasse nicht möglich ist und die medikamentöse Schmerztherapie dem WHO-Stufenschema folgen sollte [668].

Bezüglich der intraperitonealen Gabe von Lokalanästhetika [669] oder der Infiltration der port-sites [670] zeigen die Metaanalysen aus 54 bzw. 19 randomisiert kontrollierten Studien keinen Vorteil. Die kleinen messbaren Differenzen zur Standard-Schmerztherapie sind klinisch nicht evident. Weiterhin ist aufgrund der unterschiedlich angewendeten Wirkstoffe und der unscharf definierten primären Studienendpunkte ein Vergleich der verschiedenen Studien schwierig. Die Endpunkte zur gesundheitsbezogenen Lebensqualität oder zur Wiederaufnahme der Arbeit wurden in keiner der einge- 
schlossenen Studien berücksichtigt, sodass keine Empfehlung zur intraperitonealen Gabe von Lokalanästhetika oder Port-site-Infiltration gegeben werden kann.

\section{EMPFEHLUNG IIIB.14.}

Gallenblasen-Sludge ist in der Lage, gleiche Beschwerden wie Gallensteine selbst zu verursachen (akute Cholezystitis, biliäre Pankreatitis u. a.) und sollte bei Symptomatik mit einer laparoskopischen Cholezystektomie behandelt werden (B, III, starker Konsens).

\section{Kommentar:}

Gallenblasen-Sludge mit typischer Klinik im Sinne kolikartiger Beschwerden im rechten Oberbauch kann zu biliären Komplikationen wie akuter Cholezystitis, Cholangitis oder akuter Pankreatitis führen, und daher ist die Cholezystektomie wie bei normaler Cholezysto-/Choledocholithiasis hier eine Therapieoption. Zu diesem Ergebnis kommt auch eine Langzeituntersuchung über fast 10 Jahre bei Patienten mit Gallenblasen-Sludge, bei der es zumindest zu ähnlich häufigen biliären Ereignissen kam wie bei Patienten mit Cholezysto-/ Choledocholithiasis [671]. Eine kleine randomisierte Studie bei 85 Patienten mit akuter Pankreatitis unklarer Ätiologie, die jedoch nicht mittels Endosonografie untersucht worden waren, berichtete, dass die Rezidivrate nach Cholezystektomie mit $10 \%$ signifikant gegenüber $41 \%$ in der Kontrollgruppe vermindert wurde; bei $60 \%$ der operierten Patienten wurde Sludge nachgewiesen [672]. Auch weitere retrospektive Untersuchungen und Übersichtsarbeiten kommen zu dem Schluss, dass Gallenblasen-Sludge mit einer Mikrolithiasis einhergehen bzw. als Vorläufer von Konkrementen biliäre Komplikationen verursachen kann und durch Cholezystektomie diese Ereignisse verhindern werden können. Allein der Nachweis von Sludge ohne Beschwerden stellt aber keine Indikation zur Cholezystektomie dar [671, $673,674]$.

\section{EMPFEHLUNG IIIB.15.}

Gallengangverletzungen bei der Cholezystektomie sollen interdisziplinär von einem in der hepato-biliären Chirurgie erfahrenen Chirurgen und einem erfahrenen interventionellen Gastroenterologen behandelt werden (A, II, starker Konsens).

\section{Kommentar:}

Die Schwere einer Gallengangverletzung ist von verschiedenen Faktoren abhängig: der Lokalisation im Verlauf des Gallenganges, wie weit der Gang durchtrennt wurde, ob die Verletzung zu einem Substanzverlust geführt hat, eine ischämische oder mechanische Verletzung vorliegt und ob es sich um eine Eröffnung oder Okklusion (z. B. mittels Clips) handelt [675].

Eine intraoperative Gallengangverletzung tritt in 0,1-0,5\% [676 - 678] der Fälle auf. Hauptgründe für die Gallengangläsion sind mangelnde Erfahrung des Operateurs, schlechte Übersicht bei entzündlichen Veränderungen mit Blutungsneigung sowie das Nichterkennen von anatomischen Varianten. Unübersichtliche Darstellung des Überganges vom Infundibulum zum D. cysticus, exzessive Präparation seiner Einmündungsstelle in den Hauptgallengang oder blutstillende Maßnahmen bei Blutungen aus der abgerissenen A. cystica sind weitere Fehler.

Bei der offenen Cholezystektomie wird die Gallengangläsion mit einer Häufigkeit von $0,2 \%$ angegeben [676, 677]. In einer Metaanalyse über annähernd 80000 laparoskopische Cholezystektomien kam es in 0,5\% der Fälle zu Gallengangverletzungen [677]. In anderen prospektiv untersuchten Studien liegt die Rate unter $0,1 \%$ [678].

Zur Vermeidung von intraoperativen Gallengangverletzungen empfiehlt sich ein standardisiertes Vorgehen des Operateurs bei der Präparation der A. cystica und des Ductus cysticus mit eindeutiger Identifikation der Strukturen mittels „Critical view of safety“ [679]. Routinemäßig angewandte intraoperative Cholangiografie vermag die Inzidenz von intraoperativen Gallengangverletzungen nicht zu senken $[680,681]$.

Wird intraoperativ eine Gallengangverletzung bemerkt, ist deren Schwere makroskopisch und mittels intraoperativer Cholangiografie zu bestimmen. Wenn diese nicht sicher durchgeführt werden kann, ist eine subhepatische Drainage einzulegen und der Patient sollte einem erfahrenen hepato-biliären Chirurgen vorgestellt werden. Die spezielle Handhabung von Gallengangverletzungen ist spezialisierten Zentren mit ausreichender Erfahrung vorbehalten [675].

Geringfügige Gallengangverletzungen, die postoperativ evident werden, können mittels subhepatisch platzierter Drainage und zunächst konservativem Vorgehen behandelt werden. Bei unauffälligem klinischen Befund, nicht bestehender Sepsis und Sekretion der liegenden subhepatischen Drainage über $200 \mathrm{ml}$ pro Tag, ist eine elektive ERC mit Stenteinlage indiziert [675].

Verletzungen ohne Substanzverlust können durch direkte Naht über ein eingelegtes T-Drain versorgt werden. Ein durchtrennter Hauptgallengang mit Substanzverlust erfordert eine biliodigestive Anastomose.

EMPFEHLUNG IIIB.16.

Das symptomatische oder komplizierte Gallensteinleiden bei transplantierten oder immunsupprimierten Patienten sollte zur frühzeitigen chirurgischen Indikationsstellung führen, da die klinischen Symptome oftmals nur verzögert auftreten oder durch die Immunsuppression nicht zur Ausprägung kommen können (B, III, starker Konsens).

\section{Kommentar:}

Die Inzidenz von Gallensteinen nach Herztransplantation [682] oder Nierentransplantation [683] ist höher als in der Allgemeinbevölkerung. Morbidität und Letalität sind bei herztransplantierten Patienten höher, wenn die Cholezystektomie im Falle einer symptomatischen Cholezystolithiasis offen oder ungeplant durchgeführt wird [684]. Belastbare Daten, die eine Cholezystektomie bei Steinträgern vor der Transplantation empfehlen, existieren nicht. Die in der Literatur verfügbaren Studien tendieren sowohl 
zur Surveillance als auch zur frühzeitigen Indikationsstellung zur Vermeidung des komplizierten Steinleidens im Sinne einer Cholezystitis [685, 686].

Zur Nierentransplantation existieren einzelne, nicht randomisierte Studien, deren Autoren eine prophylaktische Cholezystektomie wegen der möglichen zu erwartenden Komplikationen nach der Transplantation für sinnvoll erachten [687, 688]. Prospektive Daten sowie Metaanalysen oder Reviews, auf deren Basis generell eine prophylaktische Cholezystektomie vor Nierentransplantation zu rechtfertigen wäre, existieren nicht.

Elektive abdominelle Operationen nach Lungentransplantation werden generell als sicher betrachtet [689], sodass das eher abwartende und überwachende Management von Gallenblasensteinen auch hier als sinnvoll erachtet werden kann.

\section{IIIC. Endoskopisch-interventionelle Therapie}

\section{EMPFEHLUNG IIIC. 1 .}

Symptomatische Gallengangsteine sollen behandelt werden (B, III, starker Konsens, NKLM).

Die Therapie asymptomatischer Gallengangsteine sollte erwogen werden (B, II, starker Konsens).

\section{Kommentar:}

Mehr als $50 \%$ der Patienten mit symptomatischen Gallengangsteinen werden innerhalb eines Jahres erneut symptomatisch und 25 \% entwickeln Komplikationen. Aus dieser Datenlage ergab sich in früheren Leitlinien die Behandlungsindikation, die jedoch auch bis heute nicht durch prospektive Studien abgesichert ist. Für die zweite Aussage gibt es eine neue Datenlage, wobei zwei Situationen zu unterscheiden sind:

1. Die Steine sind aus der Gallenblase in den Gallengang gewandert. Die Passage der Gallenblasensteine in den Ductus hepatocholedochus geschieht bei etwa $10-15 \%$ der Patienten. Der natürliche Verlauf dieser Gallengangsteine ist nach wie vor nicht hinreichend geklärt. Man nimmt an, dass bis zu 50\% der Gallengangsteine asymptomatisch bleiben können. Ein großer Teil dieser Steine geht entweder asymptomatisch (ca. 20\%) oder mit einer begleitenden milden, selbst limitierenden Pankreatitis ab. Diese Situation der simultanen Gallenblasen- und Gallengangsteine wird in Empfehlung IIIC.2 besprochen. Die Situation, dass zufällig sonografisch simultan asymptomatische Gallenblasensteine und asymptomatische Gallengangsteine entdeckt werden, ist selten. Zu dieser Situation gibt es keine prospektiven Studien. Der natürliche Verlauf von zufällig intraoperativ entdeckten und nicht durch therapeutisches Splitting präoperativ behandelten Gallengangsteinen bei symptomatischen Gallenblasensteinträgern ist in einer retrospektiven Analyse in der GallRiks-Studie $(n=3828)$ untersucht worden. Die Komplikationsrate (Kolik, Cholangitis, Pankreatitis) lag bei $25 \%$, wenn Steine nicht entfernt wurden, vs. $13 \%$, wenn Steine intraoperativ entfernt wurden. Die Odds Ratio war von der Steingröße abhängig, und das Komplikationsrisiko verdoppelte sich weiter, wenn die Gallengangsteine $>4$ - $8 \mathrm{~mm}$ waren (OR 0,52 bei Steingröße < 4 mm, OR 0,24 bei Steingröße 4-8 mm) [690].

2. Bei cholezystektomierten Patienten liegt eine andere Situation vor. In dieser Patientengruppe entstehen die Gallengangsteine primär im Gallengang, oder es handelt sich um nicht diagnostizierte vor der Cholezystektomie abgegangene Steine. Die primären Gallengangsteine werden häufig Infekt-getriggert durch Stase im Gallengang (juxtapapilläres Divertikel, Stenose) oder durch aufsteigende Infekte bei Z. n. weiter Papillotomie in ihrer Genese begünstigt. Die klinische Erstmanifestation dieser Gallengangsteine beinhaltet häufig (bis zu 75 \%) Komplikationen wie obstruktiven Ikterus, biliäre Pankreatitis oder Cholangitis [691]. Aus diesem Grund sollten diese Gallengangsteine ebenfalls extrahiert werden, so lange sie asymptomatisch sind.

\section{EMPFEHLUNG IIIC.2.}

Bei Patienten mit gleichzeitig vorliegenden Gallenblasen- und Gallengangsteinen sollte ein therapeutisches Splitting (präoder intraoperativ) erfolgen (B, I, starker Konsens, NKLM).

\section{Kommentar:}

Die Rechtfertigung eines therapeutischen Splittings mit präoperativer ERC hängt von der Sicherheit ab, mit der ein Gallengangstein präoperativ diagnostiziert werden kann. Die besten Verfahren, einen Gallengangstein zu diagnostizieren, sind die MRCP und die Endosonografie. Zur Indikation des therapeutischen Splittings wird im diagnostischen Teil Stellung genommen (siehe II.9).

In Deutschland hat sich bei der hohen Dichte des ERC-Angebots das therapeutische Splitting gut und erfolgreich etabliert. Die ERC kann prä-, intra- oder postoperativ durchgeführt werden. Die Verfahren sind in Metaanalysen gleichwertig, wobei die Krankenhausverweildauer bei der prä- und postoperativen ERC etwas länger ist. Retrospektive Studien im schwedischen Register für Gallenstein-Chirurgie und ERC (GallRiks) zeigen, dass das Pankreatitis-Risiko bei der intraoperativ durchgeführten ERC geringer zu sein scheint (prä-OP-ERC 3,6\% vs. intra-OP-ERC 2,2\%, OR 0,5, $P=0,02$, Number needed to treat 71) [692]. Allerdings ist die logistische Hürde in den meisten Kliniken aus organisatorischen Gründen zu hoch, sodass in diesen Kliniken das präoperative Splitting vorgezogen wird.

Bei entsprechender chirurgischer Expertise kann alternativ eine laparoskopische Cholezystektomie mit laparoskopischer Choledochusrevision durchgeführt werden, da dieses Vorgehen dem therapeutischen Splitting nicht unterlegen ist [693, 694]. Letalität, Morbidität und Konversionsraten waren in beiden Gruppen gleich groß. Daten zu Kosten, Krankenhausverweildauer und Lebensqualität konnten aufgrund fehlender Daten nicht analysiert werden. Die Steinfreiheitsrate war in der rein chirurgischen Gruppe zwar höher als bei den Patienten mit therapeutischem Splitting, der Unterschied war jedoch nicht signifikant (92 vs. $86 \%$ ) [694].

Werden die Steine erst intraoperativ entdeckt und ist die laparoskopische Choledochusrevision nicht möglich, so sollte das the- 
rapeutische Splitting durch die postoperative ERC vervollständigt werden.

\section{EMPFEHLUNG IIIC.3.}

Vor endoskopischer transpapillärer Steinextraktion soll eine Papillotomie durchgeführt werden (A, I, starker Konsens, NKLM).

Zur Erweiterung der Papillotomie kann bei großen Gallengangsteinen zur Verbesserung der Steinextraktion eine endoskopische papilläre Ballondilatation (EPBD) durchgeführt werden (0, I, starker Konsens).

\section{Kommentar:}

Das Risiko, Gallengangsteine durch eine intakte Papille zu extrahieren, ist inakzeptabel hoch und daher obsolet [695]. Eine vorherige Papillotomie soll durchgeführt werden. Alternativ zur Papillotomie wird die Ballondilatation eingesetzt. Der technische Erfolg ist der EPT vergleichbar, das Blutungsrisiko ist verringert. Allerdings war das Pankreatitis-Risiko in Metaanalysen um den Faktor 2-4 erhöht [696]. Aus diesem Grund ist die alleinige EPBD nur in begründeten Ausnahmefällen (Immundefekte, Koagulopathien, nicht absetzbare Gerinnungshemmertherapie) zulässig. Vor allem bei behebbaren Gerinnungstörungen ist die vorherige Anlage eines Stents oder einer nasobiliären Sonde bis zur Normalisierung der Gerinnungssituation eine sehr gute Alternative.

Aus Beobachtungsstudien wird jedoch ersichtlich, dass in den Jahren nach der Behandlung die Patienten, die durch Ballondilatation behandelt wurden, seltener Cholangitiden oder Steinrezidive entwickeln [697]. Dies ist vor allem für die Entwicklung von Steinrezidiven, die sich bei Patienten mit Steinextraktion nach Ballondilatation beinahe halbiert, signifikant.

Um sowohl das Blutungsrisiko nach EPT zu reduzieren als auch die Pankreatitis-Wahrscheinlichkeit nach Ballondilatation zu minimieren, wurden Studien zur kombinierten Behandlung mit Ballondilatation nach vorheriger EPT durchgeführt. Dabei waren bei einer Kombinationsbehandlung bei vergleichbarer Erfolgsrate sowohl das Pankreatitis-Risiko als auch die Blutungsrate im Vergleich zur alleinigen EPT signifikant niedriger [698, 699]. Dieses Ergebnis zeigt sich vor allem bei großen Steinen, die sich nach alleiniger EPT nur schwer extrahieren ließen. Wichtig war dabei, dass der Ballondurchmesser die Weite des Gallenganges nicht überschreiten darf, um eine Perforation zu vermeiden. Die meisten Studien verwendeten unabhängig vom Durchmesser des Gallengangs $8 \mathrm{~mm}$-Ballons, wobei längere Dilatationszeiten (> 1 $5 \mathrm{~min}$ ) sicherer waren [700]. Metaanalysen zeigen, dass die Kombination von EPT + EPBD die Notwendigkeit der mechanischen Lithotripsie verringert [701, 702]. Eine einzelne randomisierte kontrollierte Studie berichtete, dass die Kombination zur Steinextraktion genauso effektiv war wie EPT mit mechanischer Lithotripsie, aber mit weniger Komplikationen einherging (4,4 vs. 20,0\%, $\mathrm{P}=0,049, \mathrm{~N}=90$ ) [703], die Ergebnisse sollten aber durch Studien weiter belegt werden.
Zwei randomisierte Studien aus Japan untersuchten, ob das Dormia-Körbchen oder der Steinballon zur endoskopischen Steinentfernung besser geeignet sind. Für die BasketBall-Studie wurden multizentrisch insgesamt 184 Patienten mit Gallengangkonkrementen $\leq 11 \mathrm{~mm}$ randomisiert [704]. Das Hauptziel war das komplette Entfernen aller Konkremente aus dem Choledochus innerhalb von $10 \mathrm{~min}$, das bei $81 \%$ der Patienten mit Körbchen und bei $84 \%$ mit Ballon erreicht wurde; die Komplikationsrate (Blutung, Pankreatitis, Cholangitis) betrug 6,6 bzw. 11,8\%. Diese Unterschiede waren nicht signifikant. Ishiwatari et al. [705] schlossen multizentrisch 172 Patienten mit Konkrementen $\leq 10 \mathrm{~mm}$ ein. Hier wurde das Hauptzielkriterium, die komplette Konkrementextraktion (ohne Zeitlimit), mit Ballon bei $92 \%$ und mit Körbchen bei 89 \% der Patienten erreicht, sodass der Ballon nicht signifikant unterlegen war und v. a. bei Patienten mit mehr als 4 Steinen im Gallengang Vorteile hatte.

\section{EMPFEHLUNG IIIC.4.}

Bei Misslingen (auch unter Einsatz der mechanischen Lithotripsie oder der endoskopischen Ballondilatation) der endoskopischen Steinextraktion sollten als adjuvante Lithotripsieverfahren extrakorporale Stoßwellenlithotripsie (ESWL), intrakorporale Laserlithotripsie oder elektrohydraulische Lithotripsie (EHL) eingesetzt werden. Bei gleichzeitiger Cholezystolithiasis soll die chirurgische Alternative interdisziplinär besprochen werden (B, III, starker Konsens, NKLM).

\section{Kommentar:}

Sind die Steine trotz mechanischer Lithotripsie oder Einsatz der endoskopischen Ballondilatation immer noch für die Extraktion zu groß, bieten sich verschiedene Verfahren, wie die intrakorporale Laserlithotripsie, die elektrohydraulische Lithotripsie sowie die extrakorporale Stoßwellenlithotripsie (ESWL) der Gallengangsteine je nach Verfügbarkeit - zur Zerkleinerung der Steine an. Die intraduktale Lithotripsie erfolgt unter direkter optischer Sicht durch ein Cholangioskop. Dadurch werden Verletzungen der Gallengangwand vermieden. Hinsichtlich der Fragmentationseffizienz zeigten kleinere randomisierte kontrollierte Studien Vorteile der elektrohydraulischen Lithotripsie und der Laserlithotripsie gegenüber der ESWL [706 - 714]. Nach Ausschöpfung all dieser Verfahren ist die Steinfreiheit bei deutlich über $95 \%$ der Patienten zu erzielen. Im interdisziplinären Austausch sollte jedoch diese Indikation bei gleichzeitiger Cholezystolithiasis mit dem Chirurgen besprochen werden.

Ist die chirurgische Cholezystektomie mit intraoperativer Choledochusrevision möglich, sollte diese dann vorgezogen werden [694, 715]. 


\section{EMPFEHLUNG IIIC.5.}

Falls die endoskopische transpapilläre Therapie nicht gelingt und eine chirurgische Therapie nicht sinnvoll ist, sollte bei symptomatischen Patienten eine perkutan-transhepatische Gallengangstein-Behandlung durchgeführt werden (B, III, starker Konsens, NKLM).

Bei multimorbiden Patienten kann alternativ die transpapilläre Einlage einer Endoprothese erfolgen (B, I, starker Konsens).

\section{Kommentar:}

Die perkutan-transhepatische Cholangiografie (PTC) und Steinbehandlung kann bei Misslingen des endoskopisch-retrograden Vorgehens alternativ zum operativen Vorgehen bei Choledocholithiasis therapeutisch eingesetzt werden [716, 717]. Die Komplikationsraten (Hämobilie, Pneumothorax, Gallenleck in das Peritoneum) der interventionell-therapeutisch erweiterten PTC sind höher als die der ERC und EPT. Bei größeren Steinen müssen auch bei der PTC oft adjuvante Lithotripsieverfahren eingesetzt werden. Aus diesen Gründen steht die chirurgische Option gleichwertig zur Verfügung.

Bei Patienten mit einem inakzeptabel hohen Risiko ist die Endoprotheseneinlage geeignet, kurzfristig die steinbedingten Probleme (obstruktive Cholangitis, Verschlussikterus) sicher zu beseitigen [718-720].

Bei sehr alten Patienten (> 80 Jahre) oder anderen HochrisikoPatienten kann die Endoprothese als Langzeittherapie belassen werden [719]. Die Dauerversorgung mit Endoprothesen sollte allerdings wegen des Risikos der sekundären Cholangitis bei Stentverschluss und nachfolgender Sepsisgefahr auf Ausnahmen beschränkt bleiben [718, 721]. Regelmäßige Stentwechsel (alle 3 Monate) sind dann notwendig.

EMPFEHLUNG IIIC.6.

Nach erfolgreicher endoskopischer Gallengang-Sanierung sollte bei Cholezystolithiasis möglichst innerhalb von 72 Stunden cholezystektomiert werden. Eine steinfreie funktionstüchtige Gallenblase kann belassen werden (B, I, starker Konsens).

\section{Kommentar:}

Nach erfolgreicher endoskopischer Entfernung der Gallengangsteine bei simultan vorliegenden Gallenblasensteinen sollte eine Cholezystektomie möglichst bald erfolgen, es sei denn, sonografisch lassen sich in der normal kontrahierenden Gallenblase keine Steine nachweisen (siehe IIIB.1 zur sonografischen Prüfung der Gallenblasenfunktion). Das bisher oft praktizierte Verfahren, diese Patienten nach 4 - 6 Wochen zur elektiven laparoskopischen Cholezystektomie wieder einzubestellen, beruht auf einer holländischen [722] und einer asiatischen Studie [723]. Beide Studien untersuchten die Fragestellung, ob nach endoskopischer Gallengangsteinentfernung eine Cholezystektomie notwendig ist. Die Nachbeobachtungszeit betrug in der holländischen Studie zwei Jahre, in der asiatischen fünf Jahre in der Untersuchungsgruppe ohne Cholezystektomie. In der Kontrollgruppe wurde in der holländischen Studie die laparoskopische Cholezystektomie innerhalb von sechs Wochen durchgeführt. Biliäre Ereignisse traten innerhalb dieser Zeit nicht auf, während in der nicht operierten Gruppe bei $42 \%$ Komplikationen (Koliken und Cholezystitis) auftraten. In der asiatischen Studie wurde die laparoskopische Cholezystektomie im Median nach 26 Tagen (1 - 123 Tage) durchgeführt. Biliäre Ereignisse traten in dieser Gruppe bei $7 \%$ der Patienten auf. In der Beobachtungsgruppe lag die Häufigkeit der biliären Ereignisse mit $24 \%$ signifikant höher. Kürzlich sind zwei weitere randomisierte Studien erschienen, die die prophylaktische Cholezystektomie nach Gallengangsteinentfernung nochmals mit Wait-and-see ohne Cholezystektomie verglichen: In einer Studie, die nur 90 Patienten umfasste und in der Crossover zwischen beiden Armen häufig war, reduzierte die OP die Inzidenz einer nachfolgenden Cholezystitis, aber nicht einer Cholangitis [724]; in der zweiten Studie wurden 162 Patienten, die alle älter als 70 Jahre waren, randomisiert, wobei auch hier die Zahl der biliären Ereignisse (einschließlich Cholangitis) durch die elektive Cholezystektomie signifikant reduziert wurde [725].

Aufgrund der Ergebnisse der ersten Studien [722] war die zeitliche Grenze der Cholezystektomie nach ERC bei 4-6 Wochen angesetzt worden. Intention der Studien war jedoch nicht, den optimalen Zeitpunkt der ERC zu erfassen, sondern die Indikation einer Cholezystektomie nach ERC zu verifizieren. Mittlerweile wurde in zwei retrospektiven Studien [726, 727] und in einer prospektiven randomisierten Multicenterstudie [728] der optimale Zeitpunkt der Cholezystektomie nach ERC untersucht. In der randomisierten kontrollierten Studie mit 94 Patienten traten bei $28 \%$ der Patienten biliäre Koliken und bei $9 \%$ eine akute Cholezystitis auf, wenn die laparoskopische Cholezystektomie erst nach 6-8 Wochen erfolgte. Wurde die Operation dagegen in weniger als $72 \mathrm{~h}$ nach der ERC mit Steinextraktion durchgeführt, so fand sich kein derartiges Ereignis. Die Rate an biliären Pankreatitiden lag in beiden Gruppen niedrig bei $2 \%$. Die Konversionsrate und OP-Dauer unterschieden sich nicht. In der retrospektiven Studie von Schiphorst et al. [726] lag die mediane Zeit bis zur Cholezystektomie bei 7 Wochen ( 1 - 49 Wochen). In diesem Zeitraum traten bei $20 \%$ Komplikationen in Form von Cholezystitis (11\%), Gallengangsteinrezidiv (5\%), Cholangitis (2\%) oder Pankreatitis (1\%) auf. Innerhalb von 1 Woche hatten $4 \%$ und nach 3 Wochen bereits $10 \%$ diese Komplikationen entwickelt. Die kürzlich publizierte retrospektive Studie von Huang et al. [727] fand bei Patienten, die noch während des Erstaufenthaltes mit primär endoskopischer Therapie eine Cholezystektomie erhielten, im Vergleich zu Patienten mit verzögerter Cholezystektomie (innerhalb von 60 Tagen nach ERC) und Verzicht auf Cholezystektomie eine Reduktion des Risikos erneuter biliärer Ereignisse innerhalb eines 60-Tage-Zeitraums um $92 \%$.

Wegen dieser Ergebnisse lässt sich die Wartezeit von 4 - 6 Wochen nicht mehr rechtfertigen. Aufgrund der Daten der prospektiven Studie liegt der optimale Zeitpunkt einer Cholezystektomie nach ERC innerhalb von $72 \mathrm{~h}$, auf Basis der retrospektiven Studie von Schiphorst et al. [726] ließe sich auch 1 Woche noch rechtfertigen, vorausgesetzt es liegt keine Pankreatitis vor. 
- Tab.4 Atlanta-Klassifikation der akuten Pankreatitis [838].

milde Form

moderate Form

schwere Form keine Organfunktionsstörungen, keine lokalen Komplikationen

lokale Komplikationen und/oder vorübergehende Organfunktionsstörungen ( $<48 \mathrm{~h}$ )

persistierende Organfunktionsstörungen $>48 \mathrm{~h}$, berechnet nach dem modifizierten Marshall-Score* für Organfunktionsstörungen

*Modifizierter Marshall-Score [839] für Organfunktionsstörungen

\begin{tabular}{|l|l|l|l|l|l|}
\hline & 0 & 1 & 2 & 3 & 4 \\
\hline $\mathrm{PaO}_{2} / \mathrm{FiO}_{2}$ & $>400$ & $301-400$ & $201-300$ & $101-200$ & $<101$ \\
\hline Kreatinin $(\mathrm{mg} / \mathrm{dl})$ & $<1,4$ & $1,4-1,8$ & $1,9-3,5$ & $3,6-4,9$ & $>4,9$ \\
\hline RR systolisch $(\mathrm{mmHg})$ & $>90$ & $<90$ & $<90$ & $<90$ & $<90$ \\
& & Ansprechen auf Volumen & kein Ansprechen auf Volumen & $\mathrm{pH}<7,3$ & $\begin{array}{l}<\mathrm{PH}<7,2 \\
\end{array}$ \\
\hline
\end{tabular}

Bei einem Score $\geq 2$ liegt eine Organfunktionsstörung vor.

\section{EMPFEHLUNG IIIC.7.}

Bei unkomplizierter biliärer Pankreatitis und abklingender Cholestase/Pankreatitis sollte auf eine ERC verzichtet werden, wenn Endosonografie oder MRCP keinen Steinnachweis ergeben haben. Die Cholezystektomie soll dann so bald wie möglich erfolgen (A, I, starker Konsens).

\section{Kommentar:}

Bei der biliären Pankreatitis mit Cholestase/Ikterus und/oder Zeichen einer Cholangitis soll eine ERC mit Steinextraktion nach Maßgabe der klinischen Dringlichkeit erfolgen (siehe IIIC.9). Die Behandlung der biliären Pankreatitis ohne Cholangitis richtet sich nach deren Schweregrad. Die meisten Fälle der biliären Pankreatitis sind mild und heilen spontan aus. Nach Ablauf einer milden Pankreatitis sollte daher bei noch vorhandenen Gallenblasensteinen die Cholezystektomie zügig erfolgen [467, 729-731]. Diese zunächst auf retrospektiven Studien beruhende Empfehlung wurde kürzlich durch die randomisierte kontrollierte PONCHO-Studie mit 264 Patienten (Alter $\leq 68$ Jahre, ASA-Risikoklasse $\leq 3$; - Tab. 3) in den Niederlanden untermauert [470], die nach milder biliärer Pankreatitis (kein Organversagen nach $>48$ h, keine lokalen Komplikationen wie peripankreatische Nekrosen, CRP $<100 \mathrm{mg} / \mathrm{l}$, orale Ernährungsmöglichkeit und Fehlen opioider Schmerzmedikamente) die frühelektive Cholezystektomie innerhalb von $72 \mathrm{~h}$ mit der spätelektiven Cholezystektomie innerhalb von 25-30 Tagen verglich. Die frühe Operation senkte die Wiederaufnahmerate bzw. Mortalität signifikant von 17 auf $5 \%$ und das Risiko für eine erneute Pankreatitis von 9 auf $2 \%$ ohne Komplikationsraten, Konversionsraten oder Krankenhausverweildauer nachteilig zu beeinflussen. Aus diesen Daten ergibt sich die Empfehlung, dass nach einer milden biliären Pankreatitis die Cholezystektomie so bald wie möglich erfolgen soll. Dieses Vorgehen war in der PONCHOStudie zudem kosteneffektiv [732].

\section{EMPFEHLUNG IIIC.8.}

Bei schwerer Pankreatitis mit Steinnachweis im Gallengang ohne Cholangitis sollte die ERC mit endoskopischer Papillotomie $<72$ h nach Symptombeginn erfolgen. Bei gegebener Indikation zur Cholezystektomie sollte diese nach Abklingen der Pankreatitis durchgeführt werden (B, II, starker Konsens, NKLM).

\section{Kommentar:}

Die Entscheidung, ob eine milde oder schwere Verlaufsform einer Pankreatitis vorliegt, stellt sich häufig erst nach $48 \mathrm{~h}$ heraus. Der Schweregrad der akuten Pankreatitis wird nach der AtlantaKlassifikation definiert [733, 734]. Die schwere Pankreatitis ist assoziiert mit Organversagen, systemischen oder lokalen Komplikationen, wobei Organversagen, die innerhalb von $48 \mathrm{~h}$ reversibel sind, nicht zwangsläufig als schwere Pankreatitis anzusehen sind ( $\triangleright$ Tab.4). In einer neueren Metaanalyse zum optimalen Zeitpunkt einer ERC ( $<24$ vs. $<72$ h) bei biliärer Pankreatitis ohne Cholangitis zeigte sich kein Unterschied in der Letalität [405]. Daher erscheint es vernünftig, den Verlauf des Schweregrads abzuwarten (24-48 h) [735, 736]. Bei schwerer Pankreatitis ist dann nach Nachweis eines Gallengangsteines durch endoskopischen Ultraschall die ERC zügig indiziert. Es ist jedoch wichtig, dass bei gleichzeitig vorliegender Cholangitis die ERC auch bei biliärer Begleitpankreatitis so rasch wie möglich erfolgen soll.

Nach einer schweren Pankreatitis sollte die Cholezystektomie erst nach Abklingen der Pankreatitis erfolgen. Diese Studien sind vor allem bei offenen Cholezystektomien durchgeführt. Es gibt vereinzelte retrospektive Analysen zur offenen Cholezystektomie nach schwerer Pankreatitis, in denen beschrieben wird, dass Patienten mit moderater bis schwerer Pankreatitis mit einem Ranson-Score über 5 Punkte bei der späten Cholezystektomie nach über 6 Wochen besser abschneiden als die Patienten, die früh operiert werden [737, 738]. Dieselben Daten gibt es auch für die laparoskopische Cholezystektomie, wobei in beiden Verfahren keine prospektiven randomisierten Studien existieren. 


\section{EMPFEHLUNG IIIC.9.}

Bei der biliären Pankreatitis mit Cholestase/Ikterus und/oder Zeichen einer Cholangitis soll eine ERC/Papillotomie mit Steinextraktion so rasch wie möglich durchgeführt werden (A, I, starker Konsens, NKLM).

Bei Cholangitis soll die ERC/Papillotomie innerhalb von $24 \mathrm{~h}$ nach Aufnahme erfolgen (A, I, starker Konsens).

\section{Kommentar:}

Beim Vorliegen einer Cholangitis oder schweren biliären Pankreatitis ist der Wert einer möglichst raschen endoskopischen Intervention gesichert [729, 739 - 741]. Die erhöhte Letalität wird durch die Ableitung der Cholangitis gesenkt. In der Studie von Oria et al. [742], die ebenfalls frühe und elektive ERC verglich, wurden bei 72 \% der Patienten im ERC-Arm Gallengangsteine nachgewiesen und entfernt; Patienten mit Cholangitis waren ausgeschlossen, und $>90 \%$ der Patienten wurden im Verlauf des stationären Aufenthaltes cholezystektomiert. Es ergaben sich keine Unterschiede hinsichtlich Organversagen, Komplikationen und Letalität [742]. Es besteht in Anlehnung an Studien [740, 743, 744] Konsens, dass die ERC/EPT innerhalb von $24 \mathrm{~h}$ nach Aufnahme erfolgen sollte.

\section{EMPFEHLUNG IIIC. 10.}

Bei obstruktiver steinbedingter akuter Cholangitis soll unverzüglich eine Antibiotikatherapie begonnen werden. Eine endoskopische Therapie der Obstruktion (Steinextraktion oder Drainage) soll in Abhängigkeit von der Dringlichkeit so rasch wie möglich, bei Zeichen der Sepsis unverzüglich, erfolgen (A, I, Konsens).

Falls das transpapilläre Vorgehen misslingt, sollen alternative interventionelle Verfahren oder ein chirurgisches Vorgehen erwogen werden (A, II, starker Konsens).

\section{Kommentar:}

Bei der Cholangitis zeigte sich in einer randomisierten Studie ein signifikanter Vorteil des endoskopischen gegenüber dem operativen Vorgehen hinsichtlich der Komplikationen und der Letalität [743]. Deshalb gilt das endoskopische Vorgehen heute nach wie vor als Therapie der Wahl. Die endoskopische Therapie erfordert nicht in jedem Fall die Durchführung einer EPT, jedoch die Einlage einer biliären Drainage [745]. Beim Misslingen der endoskopischen Steintherapie und drohender mechanischer Obstruktion des biliären Systems sind sofortige Drainagemaßnahmen unerlässlich. Eine systemische antibiotische Therapie soll sofort angesetzt werden.

Gelingt es auf endoskopischem Weg nicht, die Papilla vateri oder den Gallengang zu erreichen, ist bei entsprechender Expertise der perkutan-transhepatische Zugang mit Anlage einer perkutan-transhepatischen Cholangio-Drainage (PTCD) angezeigt. Große Gallengangsteine können mit den gleichen Fragmentationsmethoden, ggf. unter Zuhilfenahme der Cholangioskopie, zerkleinert und entfernt werden, wie dies für den transduodenalen Zugang gilt. Die Erfolgsrate für die Behandlung von Steinen in den Gallengängen mit perkutan-transhepatischer Cholangioskopie beträgt $90-99 \%$, die Komplikationsrate ist etwas höher als bei der EPT.

In Fällen, in denen die Papille endoskopisch zwar einstellbar, jedoch nicht intubierbar ist, kann ein kombiniertes perkutanes oder endosonografisches und endoskopisches Vorgehen indiziert sein (so genanntes Rendezvous-Verfahren), bei dem ein perkutan oder durch endosonografische Feinnadelpunktion (EUS-FNP) platzierter Führungsdraht endoskopisch aufgenommen wird und als Schiene für die weitere, dann endoskopische Intervention verwendet werden kann. Misslingt die steinbedingte Behebung der Gallengangobstruktion, muss perkutan-transhepatisch interveniert werden. Steht dieses Verfahren nicht zur Verfügung, sollte der Patient in ein Zentrum verlegt werden. Eine Alternative zum perkutan-transhepatischen Vorgehen ist in Expertenhand die endosonografisch gestützte Gallengangsdrainage [746], die auf verschiedenen Zugangsrouten erfolgen kann und in einer Metaanalyse im Vergleich zur perkutan-transhepatischen Gallenwegsdrainage eine höhere klinische Effektivität, weniger Komplikationen und eine geringere Re-Interventionsrate aufwies [747]. Einschränkend muss gesagt werden, dass nach einer anderen Metaanalyse nur etwa $14 \%$ der in Studien eingeschlossenen Patienten benigne Indikationen für die Durchführung einer endosonografischen Gallengangsdrainage aufwiesen [748].

Bei obstruktiver, steinbedingter, akuter Cholangitis sind neben der unverzüglichen antibiotischen Therapie und Flüssigkeitssubstitution zur Stabilisierung der Kreislaufsituation eine rasche Steinentfernung oder eine Drainage mit Stent-Einlage oder Anlage einer nasobiliären Sonde erforderlich. Der Zeitpunkt der Obstruktionsbehandlung (Steinextraktion oder Drainage) richtet sich nach dem Schweregrad der Cholangitis.

Bei schwerer akuter Cholangitis Grad III (zur Definition siehe II.10) [342] sind eine Drainage oder Beseitigung des Hindernisses unverzüglich erforderlich; unverzüglich bedeutet in diesem Kontext alsbaldiges Handeln nach einer angemessenen Zeit weiterer diagnostischer Überlegungen. Die endoskopische Ableitung ist der chirurgischen Sanierung deutlich überlegen. Wenn die Steinextraktion misslingt, so sind eine nasobiliäre Sonde oder ein biliärer Stent zur Ableitung notwendig. Beide Verfahren sind gleich effektiv. Bei akuter Cholangitis Grad II ist die endoskopische Sanierung innerhalb von $24 \mathrm{~h}$ erforderlich [749]. Treffen Kriterien weder für Grad II noch für Grad III zu, so reicht die endoskopische Sanierung innerhalb der ersten $72 \mathrm{~h}$ aus.

\section{EMPFEHLUNG IIIC. 11.}

Eine prophylaktische Antibiotikagabe vor ERC ist nicht notwendig (A, I, starker Konsens).

Gelingt die vollständige Obstruktionsbeseitigung (Steinextraktion oder Stent) nicht im ersten Versuch, sollte eine Antibiotikaprophylaxe erfolgen (B, II, starker Konsens). 


\section{Kommentar:}

Eine prophylaktische Antibiotikatherapie vor ERC senkt nicht die Letalität und auch nicht die Cholangitis-Rate, wenn der Stein erfolgreich bei der ersten ERC komplett entfernt werden kann. Mehrere randomisierte und teilweise Placebo-kontrollierte Studien untersuchten die Wirksamkeit einer prophylaktischen Antibiose bei unselektierten Patienten oder bei Patienten mit einer Gallengangobstruktion. Diese Studien wurden in Metaanalysen [750, 751] und einer Cochrane-Analyse ausgewertet [752]. Es konnte dabei nicht gezeigt werden, dass durch eine antibiotische prophylaktische Therapie die Mortalität reduziert werden kann [752]. Es kann aus diesen Daten jedoch abgeleitet werden, dass die Cholangitis-Rate nach Misserfolg im ersten Versuch unter Antibiotikagabe signifikant um die Hälfte reduziert werden kann. Bei Patienten mit erfolgreicher Steinentfernung bei der ersten ERC besteht kein Unterschied zwischen der Cholangitis-Rate mit oder ohne prophylaktische Antibiotikagabe [752]. Die Dauer der antibiotischen Therapie in prophylaktischer Indikation ist nicht durch randomisierte Studien abgesichert.

Es sollte eine komplette Drainage aller mit Kontrastmittel dargestellten Gangsysteme angestrebt werden; zudem sollte nur in die Segmente Kontrastmittel injiziert werden, die mit einem Führungsdraht sondiert werden können und die damit auch prinzipiell mittels interventioneller Therapie drainierbar sind.

\section{EMPFEHLUNG IIIC. 12.}

Eine Cholangioskopie kann die intraduktale Lithotripsie transpapillär oder perkutan unter Sicht unterstützen (B, III, starker Konsens). Die Cholangioskopie soll unter Wasserspülung oder $\mathrm{CO}_{2}$-Insufflation durchgeführt werden (GCP, III, starker Konsens).

\section{Kommentar:}

Bei der Behandlung der Gallengangsteine kann eine Cholangioskopie die Lithotripsie unter Sicht unterstützen und die Kontrolle der Steinfreiheit nach Lithotripsie dokumentieren [753, 754]. Voraussetzung für die transpapilläre Cholangioskopie sollte eine adäquat weite Papillotomie sein. Eine periinterventionelle Antibiotikaprophylaxe wird empfohlen.

Die Visualisierung des Gallengangsystems erfolgt meist unter Wasserspülung oder $\mathrm{CO}_{2}$-Insufflation. Im Rahmen der direkten peroralen Cholangioskopie mit Luftinsufflation wurden schwerwiegende Luftembolien beschrieben [755, 756].

Mit dem Einsatz ultradünner Endoskope, die primär für die transnasale Endoskopie des oberen Gastrointestinaltrakts entwickelt wurden, ist auch die direkte perorale Cholangioskopie mit guter Bildqualität ohne ERC möglich. Nach weiter Papillotomie erfolgt eine direkte Intubation des Ductus choledochus.
EMPFEHLUNG IIIC.13.

Bei Patienten mit akuter (auch akalkulöser) Cholezystitis und hohem Operationsrisiko kann eine perkutane Drainage der Gallenblase (Cholezystostomie) erfolgen (0, II, starker Konsens).

\section{Kommentar:}

Bei akuter Cholezystitis bei älteren Patienten (> 65 Jahre) oder erhöhtem Operationsrisiko (ASA-Risikoklasse $\geq$ III; $>$ Tab. 3) kann eine perkutane Drainage der Gallenblase (Cholezystostomie) mit niedrigen Letalitäts- und hohen Erfolgsraten durchgeführt werden [757, 758]. Es liegen randomisierte kontrollierte Studien vor, bei denen die Cholezystostomie mit früher oder später Cholezystektomie [759] bzw. die Cholezystostomie mit der zunächst rein konservativen Therapie verglichen wurden [760]. Hierbei konnten im systematischen Review der randomisierten kontrollierten Studien keine Unterschiede hinsichtlich der Letalität in den verschiedenen Armen detektiert werden [761]. Die Auswertung von retrospektiven Studien konnte eine Gleichwertigkeit von Cholezystektomie und Cholezystostomie nicht belegen [762]. Ohne Cholezystektomie besteht die Gefahr, dass sich der Zustand der Patienten im Verlauf wieder verschlechtert [760].

Alternative Verfahren sind die endosonografisch gesteuerte Gallenblasendrainage (EUS-GBD) [763] und transpapilläre Gallenblasendrainagen [764, 765], die in randomisierten Studien evaluiert wurden und durch erfahrene Untersucher in spezialisierten Zentren durchgeführt werden. Die Ergebnisse für die nasobiliäre Drainage und Stenting der Gallenblase mit 6 - 10 Fr-Stents waren hinsichtlich technischer und klinischer Erfolgsraten sowie Komplikationen vergleichbar [766]. Für die EUS-GBD bei akuter Cholezystitis wurden zuletzt insbesondere Lumina-aneinanderlegende Metallstents (LAMS) erfolgreich eingesetzt [767], wobei die Komplikations- und Reinterventionsraten in retrospektiven Serien niedriger als beim perkutanen Vorgehen waren [768-770]. Die Ergebnisse scheinen der endoskopisch-transpapillären und der perkutanen Gallenblasendrainage zumindest ebenbürtig zu sein [768 - 773]. Die aktualisierte japanische Leitlinie empfiehlt bei chirurgischen Hochrisikopatienten die perkutane Drainage als Standard, sieht jedoch die EUS-GBD durch erfahrene Endoskopiker als gleichwertig evidenzbasiertes Verfahren an [766]. Es sind weitere ausreichend große Studien zu dieser Fragestellung und auch im Vergleich mit der subtotalen Cholezystektomie [774] sinnvoll.

\section{EMPFEHLUNG IIIC. 14.}

Asymptomatische intrahepatische Steine sollten nicht grundsätzlich behandelt werden (B, III, starker Konsens). Bei symptomatischen intrahepatischen Steinen sollte die Therapieentscheidung patientenbezogen interdisziplinär getroffen werden (B, IV, starker Konsens). 


\section{Kommentar:}

Asymptomatische intrahepatische Steine werden im Verlauf von 15 Jahren nur bei 11,5\% der Patienten im Mittel nach 3,4 Jahren symptomatisch [477]. Bei inpaktierten intrahepatischen Steinen ist das Risiko, durch die ERC eine Cholangitis zu induzieren, erhöht. Aus diesem Grund ist das abwartende Verhalten gerechtfertigt. Bei symptomatischen Steinen ist die interdisziplinäre Therapieplanung sinnvoll. Die häufigsten Symptome sind Koliken, Cholangitis und Leberabszess, seltener Cholangiokarzinome [477]. Aus diesem Grund ist das abwartende Verhalten gerechtfertigt. Bei symptomatischen Steinen ist die interdisziplinäre Therapieplanung sinnvoll [478]. Der Langzeiterfolg der Chirurgie ist insbesondere dann gegeben, wenn der Befall auf einzelne periphere Lebersegmente und einen Leberlappen begrenzt ist [479]. Bei diffuser Verteilung der intrahepatischen Steine sind perkutantranshepatische cholangioskopische Verfahren mit Lithotripsie und Gallengangdilatationen sinnvoll [474, 480 - 483, 775, 776].

\section{EMPFEHLUNG IIIC.15.}

Symptomatische Gallengangsteine sollten auch in der Schwangerschaft durch endoskopische Papillotomie und Steinextraktion therapiert werden (B, III, starker Konsens). Falls eine Verwendung von Röntgenstrahlen erforderlich ist, stellt diese bei vorherigem Nachweis von Gallengangsteinen unter Beachtung der Strahlenschutzrichtlinien auch im ersten Trimenon keine absolute Kontraindikation dar (Statement, IV, starker Konsens).

\section{Kommentar:}

Mehrere Studien haben die Sicherheit der ERC in der Schwangerschaft bei tendenziell erhöhtem Pankreatitisrisiko nachgewiesen [777 - 787]. Die Untersuchung sollte durch einen erfahrenen Untersucher erfolgen, wobei eine Ultraschall-geleitete EPT helfen kann, die Strahlenbelastung zu vermeiden [654, 788]. Zu Risiken und unerwünschten Arzneimittelwirkungen von Sedativa, Analgetika und Antibiotika wird auf den Kommentar zur Empfehlung IIIB.11 und die Datenbank Embryotox (htttp://www.embryotox. de/) verwiesen.

Die Ermittlung der Strahlendosis basiert auf dem 3-StufenKonzept, wobei bis 20 mSv (Stufe I) eine Dosisabschätzung und Protokollierung durch den Arzt ausreichend ist [789]; die Schwellendosis für Fehlbildungen liegt bei 100 mSv (deterministische Schäden). Da eine Durchleuchtung bei lateraler Projektion und normaler Konstitution zu einer Uterusdosis von $32 \mathrm{mSv} / \mathrm{min}$ führt, sollten die Durchleuchtungszeiten möglichst kurz gehalten und keine Röntgenaufnahmen angefertigt werden [789]. Die schwangere Patientin sollte für den Eingriff auf die linke Körperseite gelagert werden, um eine Okklusion der Vena cava zu vermeiden [654]. Bei der Stromapplikation soll die Neutralelektrode so (rechtsthorakal oder am rechten Oberarm) platziert werden, dass der Uterus sich nicht zwischen Sphinkterotom und der Elektrode befindet.

\section{Qualitätssicherung}

\section{EMPFEHLUNG IV. 1 .}

Die folgenden Qualitätsindikatoren sollten für die Cholezystektomie erfüllt werden (Zielbereiche in Klammern):

- Operationsbedingte Gallenwegskomplikationen bei Cholezystektomie oder innerhalb von 30 Tagen nach Cholezystektomie (Okklusion oder Durchtrennung des Ductus hepatocholedochus als Sentinel event)

- Reinterventionen aufgrund von Komplikationen bei Cholezystektomie oder innerhalb von 90 Tagen nach Cholezystektomie (Verhältnis der beobachteten zur erwarteten Rate $\leq 95 \%$-Perzentil aller Kliniken)

- Sterblichkeit bei Cholezystektomie oder innerhalb von 90 Tagen nach Cholezystektomie (Verhältnis der beobachteten zur erwarteten Rate $\leq 95 \%$-Perzentil aller Kliniken, Letalität in den ASA-Risikoklassen I - III als Sentinel event)

(B, III, starker Konsens).

\section{Kommentar:}

Sentinel event wird in den Begriffsdefinitionen des Leitlinienreports definiert. Die Empfehlung nimmt die Indikatoren auf, die 2015 im Bericht des AQUA-Instituts zur Prüfung und Bewertung der Indikatoren der externen stationären Qualitätssicherung für die verpflichtende öffentliche Berichterstattung empfohlen wurden (Kategorien 1 und 2) [790]. Die hier genannten Qualitätsindikatoren sind keine offiziellen Indikatoren der gesetzlichen Qualitätssicherung oder des Instituts für Qualitätssicherung im Gesundheitswesen (IQTIG). Basierend auf den Ergebnissen des AQUA-Instituts [791] ist die Fortführung des Qualitätssicherungsverfahrens Cholezystektomie durch das IQTIG geplant. Die Regelungen entsprechen dem Status des IQTIG als unabhängigem Institut nach §137a SGB V.

Intraoperative Verletzungen oder die Okklusion des Ductus hepatocholedochus sind eingriffsspezifische Komplikationen von Cholezystektomien und werden in Studien zur Beurteilung der Ergebnisqualität von Cholezystektomien genutzt. Sie sind eine der gravierendsten Komplikationen von Cholezystektomien [792]. Die Bundesauswertung zum Erfassungsjahr 2014 berichtet von 203 Fällen mit Durchtrennung oder Verschluss des Ductus hepatocholedochus (0,12\% aller Fälle) [793]. Die Verletzungen der Gallengänge bzw. der Verschluss der Gallengänge können sowohl intraoperativ als auch postoperativ entdeckt und behandelt werden [794]. Verletzungen der Gallengänge sind ein Hauptgrund für den Umstieg von einer laparoskopischen Cholezystektomie auf eine offen-chirurgische Cholezystektomie [795]. Gallengangverletzungen (siehe IIIB.15) führen in prospektiven Studien trotz sehr guter objektiver Ergebnisse der überwiegend endoskopischen Therapie zu einer signifikanten Beeinträchtigung der Lebensqualität im Langzeitverlauf $[677,796]$.

Eine Reintervention ist ein erneuter operativer oder interventioneller Eingriff nach einer Operation wegen postoperativ aufgetretener Komplikationen. Typische Gründe für eine Reintervention nach Cholezystektomie sind belassene Gallengangsteine, Gallen- 
gangverletzungen, Blutungen und Entzündungen. Bei der elektiv durchgeführten Cholezystektomie ist die Reinterventionsrate geringer als bei akuten Eingriffen [797, 798]. Ferner treten bei laparoskopischen Cholezystektomien deutlich seltener Komplikationen als bei offen-chirurgischen Operationen auf [799]. Da die Wahl des offenen Zugangs aber hauptsächlich bei Patienten mit sehr ungünstigen Voraussetzungen gewählt wird, beschreibt dies keinen Qualitätsunterschied, sondern beruht auf einem Selektionseffekt.

Der Tod innerhalb von 90 Tagen nach Cholezystektomie legt einen Einfluss der postoperativen Komplikationen auf die präfinale Entwicklung nahe. Die Vermeidung von postoperativen Komplikationen und deren angemessene Behandlung ist der wichtigste Schritt zur Vermeidung von postoperativen Todesfällen. Dieser Indikator dient der indirekten Überprüfung einer guten Diagnoseund Behandlungsqualität bei Cholezystektomien. Die Letalität aller in der letzten AQUA-Erhebung 2014 erfassten Cholezystektomien lag bei 0,90\% ( $\vee$ Tab. 5); bei Patienten der Risikoklassen ASA Risikoklassen I - III ( $\triangleright$ Tab. 3) betrug sie 0,42 \% [793].

Die oben genannten Indikatoren der Ergebnisqualität können auf der Basis von Routinedaten evaluiert werden [800]. Zwischen 2009 und 2013 starben in Deutschland 2957 Patienten bei 731000 Cholezystektomien, was einer Krankenhaussterblichkeit von $0,4 \%$ entspricht; eine höhere Sterblichkeit wurde insbesondere mit einem Alter $>65$ Jahre sowie Begleiterkrankungen (Herzinsuffizienz, Niereninsuffizienz), Gerinnungsstörungen und Mangelernährung assoziiert [801].

Für 889000 zwischen 2009 und 2014 in deutschen Krankenhäusern durchgeführte Cholezystektomien konnte kein Unterschied hinsichtlich der risikoadjustierten Letalität zwischen Kliniken mit sehr niedrigen (Median 71 pro Jahr [44-91]) und sehr hohen Fallzahlen (Median 286 pro Jahr [264 - 331]) festgestellt werden [802].

Verschiedene validierte Verfahren zur Erhebung der Lebensqualität wurden in randomisierten klinischen Studien zur Therapie von Gallensteinen verwendet ( $\triangleright$ Tab. 6) [523, $803-816]$. Die gemeinsamen Endpunkte beim Gallensteinleiden umfassen das Sistieren der Schmerzen und die Dauer der Rekonvaleszenzphase und insbesondere die Häufigkeit von Gallengangverletzungen und der mit ihnen verbundenen langfristigen Beeinträchtigung der Lebensqualität ( Tab.6). Obgleich krankheitsspezifische Fragebögen die durch Gallensteine bedingten Beschwerden erfassen, müssen sie auch auf generischen Skalen wie EQ-5D ( $\triangleright$ Tab.6) abgebildet werden, um den Einfluss auf den Gesundheitsstatus über verschiedene Krankheiten hinweg vergleichen zu können. Nur zwei Fragebögen (SF-36 und GIQLI) wurden im Hinblick auf patientenrelevante Veränderungen evaluiert (minimal clinically important difference, MCID) [817, 818]. Hier ist auch zu beachten, dass Gesundheitszustand und Lebensqualität als subjektive Einschätzung und Gesundheitszustand als medizinisches Assessment verschiedener Funktionen differenziert werden [819, 820]. Die meisten Studien fokussierten auf prä- und postoperative Schmerzen und Komplikationsraten der Eingriffe, sodass die verwendeten Skalen eher die Auswirkungen der Cholezystektomie auf den Gesundheitszustand als auf die Lebensqualität messen.
- Tab. 5 Ergebnisqualität der Cholezystektomie 2014 [793].

\begin{tabular}{|l|l|l|}
\hline Qualitätsindikator & $\begin{array}{l}\text { absolute } \\
\text { Zahlen }\end{array}$ & $\begin{array}{l}\text { relative } \\
\text { Zahlen }\end{array}$ \\
\hline $\begin{array}{l}\text { Eingriffsspezifische Komplikation: } \\
\text { Verschluss oder Durchtrennung } \\
\text { des Ductus hepatocholedochus }\end{array}$ & $203 / 176097$ & $0,12 \%$ \\
\hline $\begin{array}{l}\text { Verhältnis der beobachteten zur } \\
\text { erwarteteten Rate (O/E) an Ver- } \\
\text { schlüssen oder Durchtrennungen } \\
\text { des Ductus hepatocholedochus }\end{array}$ & $203 / 214$ & 0,94 \\
\hline $\begin{array}{l}\text { Reinterventionsrate aufgrund von } \\
\text { Komplikationen }\end{array}$ & $4516 / 176097$ & $2,56 \%$ \\
\hline $\begin{array}{l}\text { O/E an Reinterventionen } \\
\text { nach laparoskopisch begonnener } \\
\text { Operation }\end{array}$ & $1252 / 99173$ & $1,26 \%$ \\
\hline Sterblichkeit im Krankenhaus & $1582 / 176097$ & $0,90 \%$ \\
\hline O/E an Todesfällen & $1592 / 1627$ & 0,98 \\
\hline Sterblichkeit bei geringem Risiko & $209 / 176097$ & $0,12 \%$ \\
\hline
\end{tabular}

- Tab. 6 Skalen, die zur Erfassung der Lebensqualität beim Gallensteinleiden eingesetzt werden können [6].

generische Skalen zur Erfassung der Lebensqualität

- EQ-5D

- 6-Item Short-Form Health Survey

- Nottingham Health Profile

- Psychological General Well-Being Index

spezifische Skalen zur Erfassung der Lebensqualität

- Gastrointestinal QOL Index

- Gallstone Impact Checklist

- Otago Gallstones Condition-Specific Questionnaire

Aktuell gibt es kein systematisches Review zur Lebensqualität nach Cholezystektomie. Zahlreiche Studien haben eine durchschnittliche Verbesserung der gesundheitsbezogenen Lebensqualität nachgewiesen [804, 806, 808, 811, 821]; während einige Studien dies nicht zeigen konnten [812].

EMPFEHLUNG IV.2.

Bei einem Gallenblasenkarzinom sind die Meldepflichten zur Dokumentation im zuständigen Krebsregister bzw. Tumorzentrum zu beachten (Statement, IV, starker Konsens).

\section{Kommentar:}

Im Rahmen der Cholezystektomie, die ursprünglich durch das Gallensteinleiden indiziert war, kann es zur Entdeckung vormals nicht diagnostizierter Gallenblasenkarzinome kommen. Diese 
- Tab.7 Zukünftige Forschungsfelder (Auswahl).

- Identifizierung genetischer und exogener lithogener Risikofaktoren sowie neuer Präventionsstrategien

- prospektive Untersuchung der genetischen und klinischen Kriterien hereditärer Gallensteinerkrankungen

- langfristiger Nutzen und Kosteneffektivität von Endosonografie vs. MRCP bei V. a. Gallengangsteine

- Kosten-Nutzen-Analysen bei Patienten mit asymptomatischen/ wenig symptomatischen Gallenblasensteinen: natürlicher Verlauf vs. laparoskopische Cholezystektomie

- systematische Analyse der Lebensqualität nach operativer Therapie des Gallensteinleidens

- Versorgungsforschung zu Langzeitresultaten der Cholezystektomie und regional unterschiedlichen Eingriffshäufigkeiten

- Studien zum Risiko biliärer Koliken und Komplikationen von asymptomatischen Gallensteinen und Gallenblasen-Sludge

- Pathogenese und Prävention von (rezidivierenden) Gallengangsteinen, Studien zur bakteriellen Besiedlung und Entzündung der Gallengänge sowie zur Antibiotikaprophylaxe

- Versorgungsforschung zur Frage der einzeitigen intraoperativen Choledochusrevision vs. therapeutisches Splitting

werden ebenso wie alle anderen Cholangiokarzinome in den epidemiologischen Krebsregistern erfasst, die durch Landesgesetze geregelt sind. Die Landesgesetze sind hinsichtlich der Übermittlung und der Daten-Speicherung sowie der Widerspruchsrechte uneinheitlich. Im Falle eines präoperativ nicht diagnostizierten Gallenblasenkarzinoms besteht die Möglichkeit, dieses zusätzlich im Zentralregister Okkultes Gallenblasenkarzinom, das durch die Deutsche Gesellschaft für Allgemein- und Viszeralchirurgie unterstützt wird, zu melden [626, 822].

\section{EMPFEHLUNG IV.3.}

Als Qualitätsindikatoren für die Endosonografie bei pankreatobiliären Fragestellungen werden empfohlen: Dokumentation der maximalen Gallengang- und Pankreasgangweite sowie intraduktaler Strukturen (B, III, starker Konsens).

Bei einer Endosonografie des biliären Systems sollte eine komplette Beurteilung des extrahepatischen Gallengangverlaufs von der Papille bis in den Leberhilus sowie der Gallenblase erfolgen (B, III, starker Konsens).

Die endosonografische Untersuchung des biliären Systems kann sowohl mit radialen als auch mit longitudinalen Echoendoskopen erfolgen (0, III, starker Konsens).

\section{Kommentar:}

Die endosonografische Untersuchung des biliären Systems soll von Untersuchern vorgenommen werden, die qualifizierte Kenntnisse und klinisch-praktische Erfahrungen in der biliären Endosonografie erworben haben. Eine entsprechende Ausbildung in einem zertifizierten Kurssystem ist grundsätzlich wünschenswert. Zur Lernkurve der Endosonografie liegen Daten vor, die sich nicht spezifisch auf das biliäre System beziehen. Die Lernkurven sind individuell variabel und legen nahe, dass die meisten Untersucher ausreichende Kompetenz nach mindestens 225 endosonografischen Untersuchungen erworben haben [823, 824].

Die S2k-Leitlinie Qualitätsanforderungen in der Endoskopie spezifiziert die Qualitätsindikatoren für die Endosonografie bei pankreatobiliären Fragestellungen wie folgt: Visualisierung des Verlaufs des Ductus hepatocholedochus und Dokumentation des maximalen Durchmessers, Visualisierung des gesamten Pankreas mit Verlauf des Ductus pancreaticus und dessen maximalem Durchmesser [435].

Die überwiegende Anzahl von Studien zum Stellenwert der Endosonografie bei biliären Fragestellungen sind mit radialen Echoendoskopen durchgeführt worden. Mit longitudinalen Echoendoskopen können Gallengangsteine mit der gleichen hohen Genauigkeit diagnostiziert werden wie mit radialen Instrumenten [143, 260, 270 - 273, 275]. In Zentren mit hoher Fallzahl ist bei pankreatobiliären Fragestellungen ein Trend hin zum Einsatz des longitudinalen Echoendoskops dokumentiert [825].

\section{EMPFEHLUNG IV.4.}

Als Qualitätsindikatoren für die ERC bei Choledocholithiasis werden empfohlen (Zielbereiche in Klammern):

a) präprozedural

- Frequenz der Antibiotikaprophylaxe bei gegebener Indikation (100\%)

- Frequenz der Post-ERC-Pankreatitis-Prophylaxe bei Risikopatienten/Risikointerventionen (100\%)

b) intraprozedural

- Frequenz des Kanülierungserfolgs für die Gallengänge bei naiver Papille und normaler Ganganatomie (>90\%)

- Frequenz der erfolgreichen Steinentfernung bei Gallengangsteinen unter $10 \mathrm{~mm}$ und normaler Ganganatomie (>90\%)

- Frequenz der Erfassung von Durchleuchtungszeit und Dosis (100\%)

c) postprozedural

- Frequenz der Post-ERC-Pankreatitis (möglichst niedrig)

- Frequenz von Blutungen nach Papillotomie (möglichst niedrig)

- Frequenz von Perforationen (möglichst niedrig)

(B, III, Konsens).

\section{Kommentar:}

Der Expertenkonsensus wurde von der Leitlinie Qualitätsanforderungen in der gastrointestinalen Endoskopie übernommen [435]. Die spezifischen Indikationen zur Antibiotikaprophylaxe werden im Kommentar zur Empfehlung IIIA.4 genannt. Zur PostERC-Pankreatitis-Prophylaxe ist die rektale Applikation von Diclofenac oder Indometacin unmittelbar vor oder nach der ERC mit Papillotomie gut belegt und soll standardgemäß angewandt werden (siehe IIIC.13).

Eine große Metaanalyse analysierte aus über 8000 Publikationen zum Thema der ERC-Qualitätskriterien 52 qualitativ adäquate prospektive und retrospektive Studien [826]. Die kumulative Erfolgsrate der Gallengangkanülierung betrug 89\% (95\% Konfi- 
denzintervall $0,87-0,92)$ und die Steinextraktionsrate aus dem Ductus hepatocholedochus lag bei 88 \% (95\% Konfidenzintervall $0,83-0,94)$. Für die Teilnahme eines Trainees an der Prozedur bei Gallengangkanülierung und Steinextraktion zeigten sich in den Studien keine Unterschiede. Die aktuelle Publikation der American Society for Gastrointestinal Endoscopy zu Qualitätsindikatoren in der ERC [827] benennt ähnliche intraprozedurale Qualitätsindikatoren, wobei hier Erfolgsraten für Gallangangkanülierung und Steinextraktion $>90 \%$ angegeben werden. Die intraprozeduralen Qualiätsindikatoren sind als vorläufig bewertete Empfehlungen anzusehen, da eine abschließende Bewertung erst nach zusätzlich erforderlichen Tests erfolgen kann. Die Erfassung der Durchleuchtungszeit und Strahlendosis soll erfolgen und ist im Strahlenschutzgesetz verankert [828].

Neben allgemeinen postprozeduralen Qualitätsindikatoren kann die Erfassung der spezifischen Komplikationen nach ERC als Qualitätsindikator dienen. Bei der Erfassung der Post-ERC-Pankreatitis-Rate sollte die Analyse auf der Indikation der Intervention basieren (z. B. naive vs. vorgeschnittene Papille, Zweiteingriff nach Precut), um Risikosubguppen zu definieren [435].

\section{EMPFEHLUNG IV.5.}

Die endoskopische Papillotomie bei Choledocholithiasis kann bei ausgewählten Patienten ambulant durchgeführt werden (B, III, starker Konsens).

Bei der Entscheidung zur ambulanten EPT sind prozedurenbezogene, patientenindividuelle und soziale Umfeldfaktoren bedeutend (Statement, IV, starker Konsens).

\section{Kommentar:}

Mehrere Studien haben gezeigt, dass Patienten unter bestimmten Voraussetzungen direkt nach einer ERC und sogar nach EPT entlassen werden können. In der Regel wird eine stationäre Nachbeobachtung von $24 \mathrm{~h}$ empfohlen, da die meisten Komplikationen nach EPT erst nach $4-24 \mathrm{~h}$ auftreten [829, 830]. In einer prospektiven Multicenter-Studie betrug die stationäre Wiederaufnahmerate nach ambulanter EPT 5,7\% [830]. Bei Vorliegen spezifischer Risikofaktoren (Sphinkter-Oddi-Dysfunktion, jüngeres Lebensalter, schwierige Kanülierung, Precut-Sphinkterotomie, Pankreasgangdarstellung, kombiniertes endoskopischperkutanes Vorgehen, Leberzirrhose) stieg diese Rate auf 12,2\% an, wohingegen sie ohne diese Risikofaktoren bei 4,2 \% lag [830].

\section{EMPFEHLUNG IV.6.}

Die elektive laparoskopische Cholezystektomie kann bei ausgewählten Patienten ambulant durchgeführt werden (0, II, starker Konsens).

Bei der Entscheidung zur ambulanten laparoskopischen Cholezystektomie sind prozedurenbezogene, patientenindividuelle und soziale Umfeldfaktoren bedeutend (Statement, IV, starker Konsens).

\section{Kommentar:}

Zu dieser Fragestellung liegen randomisierte kontrollierte Studien vor [751], es sind jedoch weitere Studien zu den Auswirkungen auf Lebensqualität und Outcome erforderlich. In der randomisierten kontrollierten Studie aus Schweden [831] unterschieden sich Komplikationsraten und Lebensqualitätsindex nicht zwischen ambulanten Patienten und stationären Kurzliegern $(\mathrm{N}=100)$; die Kosten wurden kaum gesenkt. Abhängig von den Studienkriterien kommen bis zu 40-60\% aller Elektivpatienten für eine tageschirurgische Behandlung in Betracht [832 - 834]. Bei etwa 80 \% der Patienten ist die Durchführung dann tatsächlich ambulant möglich; die Wiederaufnahmerate ist mit 3,4\% vertretbar gering [835]. Beachtenswert ist, dass ein hoher Anteil der Patienten (20 - $32 \%$ ) retrospektiv eine stationäre Behandlung vorgezogen hätte [832, 836, 837]. Voraussetzung für eine ambulante Cholezystektomie sind neben patientenspezifischen Faktoren eine sorgfältige präoperative Vorbereitung und Aufklärung sowie strukturierte Nachsorgepläne.

\section{Tabellen}

- Tab. 1:Kriterien für eine simultane Choledocholithiasis bei Cholezystolithiasis

- Tab. 2: Dosierung von Antibiotika in der perioperativen Prophylaxe (aus: [542])

- Tab. 3: ASA-Klassifikation

- Tab. 4: Atlanta-Klassifikation der akuten Pankreatitis [838]

- Tab. 5: Qualitätsindikatoren für die Cholezystektomie 2014 [793]

- Tab. 6: Skalen, die zur Erfassung der Lebensqualität beim Gallensteinleiden eingesetzt werden können [6]

- Tab. 7: Zukünftige Forschungsfelder (Auswahl) 17

\section{Abkürzungen}

$\begin{array}{ll}\text { ABC } & \text { ATP Binding Cassette } \\ \text { ACDC } & \begin{array}{l}\text { Acute Cholecystitis: Early vs. Delayed } \\ \text { Cholecystectomy }\end{array} \\ \text { ALT } & \text { Alanin-Aminotransferase } \\ \text { AP } & \text { alkalische Phosphatase } \\ \text { ASA } & \text { American Society of Anesthesiologists } \\ \text { AST } & \text { Aspartat-Aminotransferase } \\ \text { BMI } & \text { Body-Mass-Index } \\ \text { CEUS } & \text { kontrastverstärkte Ultraschall-Untersuchung } \\ \text { CHOLEGAS } & \text { Gastrectomy Plus Prophylactic Cholecystec- } \\ & \text { tomy in Gastric Cancer Surgery } \\ \text { CRP } & \text { C-reaktives Protein } \\ \text { CT } & \text { Computertomografie } \\ \text { DMPK } & \text { Dystrophia myotonica protein kinase } \\ \text { EHL } & \text { elekrohydraulische Lithotripsie } \\ \text { EPBD } & \text { endoskopische Papilläre Ballondilatation }\end{array}$




$\begin{array}{ll}\text { EPT } & \text { endoskopische Papillotomie } \\ \text { ERC } & \text { endoskopisch-retrograde Cholangiografie } \\ \text { ESBL } & \text { Extended Spectrum } \beta \text {-Laktamasen } \\ \text { ESWL } & \text { extrakorporale Stoßwellenlithotripsie } \\ \text { EUS-FNP } & \begin{array}{l}\text { endosonographisch gesteuerte Feinnadel- } \\ \text { punktion }\end{array} \\ \text { EUS-GBD } & \begin{array}{l}\text { endosonographisch gesteuerte Gallenblasen- } \\ \text { drainage }\end{array} \\ \text { HERS } & \text { Heart and Estrogen Replacement Study } \\ \text { KM } & \text { Kontrastmittel } \\ \text { LAMS } & \text { Lumina-aneinanderlegende Metallstents } \\ \text { LPAC } & \text { Low phospholipid-associated cholelithiasis } \\ \text { MRCP } & \text { Magnetresonanzcholangiografie } \\ \text { MRT } & \text { Magnetresonanztomografie } \\ \text { MTBE } & \text { Methyl-Ter-Butyl-Ether } \\ \text { NCCN } & \text { National Comprehensive Cancer Network } \\ \text { NKLM } & \text { nationaler Kompetenz-basierter Lernziel- } \\ & \text { katalog Medizin } \\ \text { NNT } & \text { Number needed to treat } \\ \text { NOTES } & \text { Natural Orifice Transluminal Endoscopic } \\ & \text { Surgery } \\ \text { NSAID } & \text { nichtsteroidale Antiphlogistika } \\ \text { NSAR } & \text { nichtsteroidale Antirheumatika } \\ \text { PEP } & \text { Postexpositionsprophylaxe } \\ \text { PONCHO } & \text { Same-admission versus interval cholecys- } \\ \text { PTC } & \begin{array}{l}\text { tectomy for mild gallstone pancreatitis } \\ \text { perkutan-transhepatische Cholangiografie }\end{array} \\ \text { SEER } & \text { Surveillance, Epidemiology and End Results } \\ \text { SILS } & \text { Program } \\ \text { UDCA } & \text { Single-incision laparoscopic surgery } \\ \text { Ursodeoxycholsäure } \\ & \\ & \end{array}$

Interessenkonflikt

Die potenziellen Interessenkonflikterklärungen und deren Bewertungen sind im Leitlinienportal dargestellt.

\section{Literatur}

[1] Arbeitsgemeinschaft der Wissenschaftlichen Medizinischen Fachgesellschaften (AWMF Online). Interdisziplinäre Leitlinie der Qualität S3 zur „Prävention und Therapie der Adipositas“. 2014. Registernummer 050-001. Deutsche Adipositas-Gesellschaft (DAG) e. V., Deutsche Diabetes Gesellschaft (DDG), Deutsche Gesellschaft für Ernährung (DGE) e. V., Deutsche Gesellschaft für Ernährungsmedizin (DGEM) e.V

[2] Lemmens VE, Oenema A, Klepp KI et al. A systematic review of the evidence regarding efficacy of obesity prevention interventions among adults. Obes Rev 2008; 9: 446-455

[3] Lombard CB, Deeks AA, Teede HJ. A systematic review of interventions aimed at the prevention of weight gain in adults. Public Health Nutr 2009; 12: $2236-2246$

[4] Brown T, Avenell A, Edmunds LD et al. Systematic review of long-term lifestyle interventions to prevent weight gain and morbidity in adults. Obes Rev 2009; 10: 627-638
[5] Enwald HP, Huotari ML. Preventing the obesity epidemic by second generation tailored health communication: an interdisciplinary review. J Med Internet Res 2010; 12: e24

[6] Lammert F, Gurusamy K, Ko CW et al. Gallstones. Nat Rev Dis Primers 2016; 2: 16024

[7] Leitzmann MF, Giovannucci EL, Rimm EB et al. The relation of physical activity to risk for symptomatic gallstone disease in men. Ann Intern Med 1998; 128: 417-425

[8] Kolotkin RL, Crosby RD, Williams GR. Health-related quality of life varies among obese subgroups. Obes Res 2002; 10: 748-756

[9] Dansinger ML, Gleason JA, Griffith JL et al. Comparison of the Atkins, Ornish, Weight Watchers, and Zone diets for weight loss and heart disease risk reduction: a randomized trial. JAMA 2005; 293: 43 - 53

[10] Storti KL, Brach JS, FitzGerald S] et al. Physical activity and decreased risk of clinical gallstone disease among post-menopausal women. Prev Med 2005; 41: $772-777$

[11] Berg A, Berg A, Frey I et al. Exercise based lifestyle intervention in obese adults: results of the intervention study m.o.B.I.L.I.s. Dtsch Arztebl Int 2008; 105: $197-203$

[12] Hu FB, Li TY, Colditz GA et al. Television watching and other sedentary behaviors in relation to risk of obesity and type 2 diabetes mellitus in women. JAMA 2003; 289: 1785-1791

[13] Saris WH, Blair SN, van Baak MA et al. How much physical activity is enough to prevent unhealthy weight gain? Outcome of the IASO 1st Stock Conference and consensus statement. Obes Rev 2003; 4: 101 114

[14] Asikainen TM, Kukkonen-Harjula K, Miilunpalo S. Exercise for health for early postmenopausal women: a systematic review of randomised controlled trials. Sports Med 2004; 34: $753-778$

[15] Kay S], Fiatarone Singh MA. The influence of physical activity on abdominal fat: a systematic review of the literature. Obes Rev 2006; 7 : $183-200$

[16] Malik VS, Schulze MB, Hu FB. Intake of sugar-sweetened beverages and weight gain: a systematic review. Am J Clin Nutr 2006; 84: 274-288

[17] Vartanian LR, Schwartz MB, Brownell KD. Effects of soft drink consumption on nutrition and health: a systematic review and metaanalysis. Am J Public Health 2007; 97: 667-675

[18] McTiernan A, Sorensen B, Irwin ML et al. Exercise effect on weight and body fat in men and women. Obesity (Silver Spring) 2007; 15: $1496-$ 1512

[19] Waller K, Kaprio J, Kujala UM. Associations between long-term physical activity, waist circumference and weight gain: a 30-year longitudinal twin study. Int J Obes (Lond) 2008; 32: 353 - 361

[20] Harland JI, Garton LE. Whole-grain intake as a marker of healthy body weight and adiposity. Public Health Nutr 2008; 11: 554-563

[21] Savage JS, Marini M, Birch LL. Dietary energy density predicts women's weight change over 6 y. Am J Clin Nutr 2008; 88: 677-684

[22] Donnelly JE, Blair SN, Jakicic JM et al. American college of sports medicine position stand. Appropriate physical activity intervention strategies for weight loss and prevention of weight regain for adults. Med Sci Sports Exerc 2009; 41: 459-471

[23] Marmot M, Atinmo T, Byers T et al. Food, nutrition, physical activity, and the prevention of cancer: a global perspective (PDF). In: 2. Washington DA ed, World Cancer Research Fund/American Institute for Cancer Research (AICR) Expert Reports. 2007

[24] Bes-Rastrollo M, van Dam RM, Martinez-Gonzalez MA et al. Prospective study of dietary energy density and weight gain in women. Am J Clin Nutr 2008; 88: $769-777$

[25] Hooper L, Abdelhamid A, Moore HJ et al. Effect of reducing total fat intake on body weight: systematic review and metaanalysis of randomised controlled trials and cohort studies. BMJ 2012; 345: e7666 
[26] Tsai C], Leitzmann MF, Willett WC et al. Fruit and vegetable consumption and risk of cholecystectomy in women. Am J Med 2006; 119: 760 - 767

[27] Nordenvall C, Oskarsson V, Wolk A. Fruit and vegetable consumption and risk of cholecystectomy: a prospective cohort study of women and men. Eur J Nutr 2018; 57: 75-81

[28] Tsai C], Leitzmann MF, Willett WC et al. Glycemic load, glycemic index, and carbohydrate intake in relation to risk of cholecystectomy in women. Gastroenterology 2005; 129: 105-112

[29] Tsai C], Leitzmann MF, Willett WC et al. Dietary carbohydrates and glycaemic load and the incidence of symptomatic gall stone disease in men. Gut 2005; 54 : $823-828$

[30] Misciagna G, Centonze S, Leoci C et al. Diet, physical activity, and gallstones - a population-based, case-control study in southern Italy. Am J Clin Nutr 1999; 69: 120-126

[31] Tsai C], Leitzmann MF, Willett WC et al. Long-term intake of dietary fiber and decreased risk of cholecystectomy in women. Am J Gastroenterol 2004; 99: $1364-1370$

[32] Lander EM, Wertheim BC, Koch SM et al. Vegetable protein intake is associated with lower gallbladder disease risk: Findings from the women's health initiative prospective cohort. Prev Med 2016; 88: 20-26

[33] Pixley F, Mann J. Dietary factors in the aetiology of gall stones: a case control study. Gut 1988; 29: 1511-1515

[34] Pixley F, Wilson D, McPherson K et al. Effect of vegetarianism on development of gall stones in women. Br Med J (Clin Res Ed) 1985; 291: 11 12

[35] Walcher T, Haenle MM, Mason RA et al. The effect of alcohol, tobacco and caffeine consumption and vegetarian diet on gallstone prevalence. Eur J Gastroenterol Hepatol 2010; 22: 1345-1351

[36] Nervi F, Covarrubias C, Bravo P et al. Influence of legume intake on biliary lipids and cholesterol saturation in young Chilean men. Identification of a dietary risk factor for cholesterol gallstone formation in a highly prevalent area. Gastroenterology 1989; 96: 825-830

[37] McConnell T], Appleby PN, Key TJ. Vegetarian diet as a risk factor for symptomatic gallstone disease. Eur J Clin Nutr 2017; 71: 731 - 735

[38] Key T], Davey GK, Appleby PN. Health benefits of a vegetarian diet. Proc Nutr Soc 1999; 58: $271-275$

[39] Barre A, Gusto G, Cadeau C et al. Diet and risk of cholecystectomy: a prospective study based on the French E3N cohort. Am J Gastroenterol 2017; 112: 1448 - 1456

[40] Tsai C], Leitzmann MF, Hu FB et al. Frequent nut consumption and decreased risk of cholecystectomy in women. Am J Clin Nutr 2004; 80: 76 81

[41] Tsai C], Leitzmann MF, Willett WC et al. The effect of long-term intake of cis unsaturated fats on the risk for gallstone disease in men: a prospective cohort study. Ann Intern Med 2004; 141: 514- 522

[42] Tsai C], Leitzmann MF, Willett WC et al. Long-term intake of trans-fatty acids and risk of gallstone disease in men. Arch Intern Med 2005; 165 : $1011-1015$

[43] Tsai C], Leitzmann MF, Willett WC et al. Long-chain saturated fatty acids consumption and risk of gallstone disease among men. Ann Surg 2008; 247: $95-103$

[44] Ortega RM, Fernandez-Azuela M, Encinas-Sotillos A et al. Differences in diet and food habits between patients with gallstones and controls. J Am Coll Nutr 1997; 16: 88-95

[45] Simon JA, Hudes ES. Serum ascorbic acid and gallbladder disease prevalence among US adults: the Third National Health and Nutrition Examination Survey (NHANES III). Arch Intern Med 2000; 160: 931-936

[46] Simon JA, Hudes ES. Serum ascorbic acid and other correlates of gallbladder disease among US adults. Am J Public Health 1998; 88: 1208 1212

[47] Simon JA, Grady D, Snabes MC et al. Ascorbic acid supplement use and the prevalence of gallbladder disease. Heart \& Estrogen-Progestin
Replacement Study (HERS) Research Group. J Clin Epidemiol 1998; 51: $257-265$

[48] Walcher T, Haenle MM, Kron M et al. Vitamin C supplement use may protect against gallstones: an observational study on a randomly selected population. BMC Gastroenterol 2009; 9: 74

[49] Tsai C], Leitzmann MF, Willett WC et al. Long-term effect of magnesium consumption on the risk of symptomatic gallstone disease among men. Am J Gastroenterol 2008; 103: 375-382

[50] Zhang YP, Li WQ, Sun YL et al. Systematic review with metaanalysis: coffee consumption and the risk of gallstone disease. Aliment Pharmacol Ther 2015; 42: $637-648$

[51] Nordenvall C, Oskarsson V, Wolk A. Inverse association between coffee consumption and risk of cholecystectomy in women but not in men. Clin Gastroenterol Hepatol 2015; 13: 1096 - 1102.e1091

[52] Leitzmann MF, Willett WC, Rimm EB et al. A prospective study of coffee consumption and the risk of symptomatic gallstone disease in men. Jama 1999; 281: 2106-2112

[53] Leitzmann MF, Stampfer MJ, Willett WC et al. Coffee intake is associated with lower risk of symptomatic gallstone disease in women. Gastroenterology 2002; 123: $1823-1830$

[54] Jørgensen T. Gall stones in a Danish population. Relation to weight, physical activity, smoking, coffee consumption, and diabetes mellitus. Gut 1989; 30: $528-534$

[55] Misciagna G, Leoci C, Guerra V et al. Epidemiology of cholelithiasis in southern Italy. Part II: Risk factors. Eur J Gastroenterol Hepatol 1996; 8: $585-593$

[56] Ruhl CE, Everhart JE. Association of coffee consumption with gallbladder disease. Am J Epidemiol 2000; 152: 1034-1038

[57] Ishizuk H, Eguchi H, Oda T et al. Relation of coffee, green tea, and caffeine intake to gallstone disease in middle-aged Japanese men. Eur J Epidemiol 2003; 18: $401-405$

[58] Attili AF, Scafato E, Marchioli R et al. Diet and gallstones in Italy: the cross-sectional MICOL results. Hepatology 1998; 27: 1492 - 1498

[59] Gebhard RL, Prigge WF, Ansel HJ et al. The role of gallbladder emptying in gallstone formation during diet-induced rapid weight loss. Hepatology $1996 ; 24: 544-548$

[60] Festi D, Colecchia A, Orsini M et al. Gallbladder motility and gallstone formation in obese patients following very low calorie diets. Use it (fat) to lose it (well). Int J Obes Relat Metab Disord 1998; 22: 592 - 600

[61] Stokes CS, Gluud LL, Casper M et al. Ursodeoxycholic acid and diets higher in fat prevent gallbladder stones during weight loss: a metaanalysis of randomized controlled trials. Clin Gastroenterol Hepatol 2014; 12: 1090 - 1100.e1092; quiz e1061

[62] Moran S, Milke P, Rodriguez-Leal G et al. Ref: Gallstone formation in obese subjects undergoing a weight reduction diet. Int J Obes Relat Metab Disord 1998; 22: 282-284

[63] Mendez-Sanchez N, Gonzalez V, Aguayo P et al. Fish oil (n-3) polyunsaturated fatty acids beneficially affect biliary cholesterol nucleation time in obese women losing weight. J Nutr 2001; 131: 2300-2303

[64] Berr F, Holl J, Jungst D et al. Dietary N-3 polyunsaturated fatty acids decrease biliary cholesterol saturation in gallstone disease. Hepatology 1992; 16: $960-967$

[65] Jonkers IJ, Smelt AH, Ledeboer M et al. Gall bladder dysmotility: a risk factor for gall stone formation in hypertriglyceridaemia and reversal on triglyceride lowering therapy by bezafibrate and fish oil. Gut 2003; 52: $109-115$

[66] Utter AC, Whitcomb DC, Nieman DC et al. Effects of exercise training on gallbladder function in an obese female population. Med Sci Sports Exerc 2000; 32: $41-45$

[67] Ko CW, Napolitano PG, Lee SP et al. Physical activity, maternal metabolic measures, and the incidence of gallbladder sludge or stones during pregnancy: a randomized trial. Am J Perinatol 2014; 31: 39-48 
[68] Kan HP, Guo WB, Tan YF et al. Statin use and risk of gallstone disease: A metaanalysis. Hepatol Res 2014. doi:10.1111/hepr.12433

[69] Wu BU, Pandol S], Liu IL. Simvastatin is associated with reduced risk of acute pancreatitis: findings from a regional integrated healthcare system. Gut 2015; 64: $133-138$

[70] Venneman NG, Renooij W, Rehfeld JF et al. Small gallstones, preserved gallbladder motility, and fast crystallization are associated with pancreatitis. Hepatology 2005; 41: $738-746$

[71] Stein A, Hermoni D, Elis A et al. Effect of ezetimibe on the prevalence of cholelithiasis. World J Gastroenterol 2012; 18: 5789-5792

[72] Wang $\mathrm{HH}$, Portincasa P, Mendez-Sanchez $\mathrm{N}$ et al. Effect of ezetimibe on the prevention and dissolution of cholesterol gallstones. Gastroenterology 2008; 134: $2101-2110$

[73] Maclure KM, Hayes KC, Colditz GA et al. Weight, diet, and the risk of symptomatic gallstones in middle-aged women. N Engl J Med 1989; 321: $563-569$

[74] Völzke H, Baumeister SE, Alte D et al. Independent risk factors for gallstone formation in a region with high cholelithiasis prevalence. Digestion 2005; 71: $97-105$

[75] Tsai C], Leitzmann MF, Willett WC et al. Central adiposity, regional fat distribution, and the risk of cholecystectomy in women. Gut 2006; 55: $708-714$

[76] Leitzmann MF, Rimm EB, Willett WC et al. Recreational physical activity and the risk of cholecystectomy in women. N Engl J Med 1999; 341 : $777-784$

[77] Broomfield PH, Chopra R, Sheinbaum RC et al. Effects of ursodeoxycholic acid and aspirin on the formation of lithogenic bile and gallstones during loss of weight. N Engl J Med 1988; 319: 1567-1572

[78] Liddle RA, Goldstein RB, Saxton J. Gallstone formation during weightreduction dieting. Arch Intern Med 1989; 149: 1750-1753

[79] Yang H, Petersen GM, Roth MP et al. Risk factors for gallstone formation during rapid loss of weight. Dig Dis Sci 1992; 37: 912 - 918

[80] Weinsier RL, Wilson L], Lee J. Medically safe rate of weight loss for the treatment of obesity: a guideline based on risk of gallstone formation. Am J Med 1995; 98: 115-117

[81] Syngal S, Coakley EH, Willett WC et al. Long-term weight patterns and risk for cholecystectomy in women. Ann Intern Med 1999; 130: 471 477

[82] Tsai C], Leitzmann MF, Willett WC et al. Weight cycling and risk of gallstone disease in men. Arch Intern Med 2006; 166: 2369-2374

[83] Shiffman ML, Sugerman HJ, Kellum JH et al. Gallstones in patients with morbid obesity. Relationship to body weight, weight loss and gallbladder bile cholesterol solubility. Int J Obes Relat Metab Disord 1993; 17 : $153-158$

[84] Sugerman HJ, Brewer WH, Shiffman ML et al. A multicenter, placebocontrolled, randomized, double-blind, prospective trial of prophylactic ursodiol for the prevention of gallstone formation following gastric-bypass-induced rapid weight loss. Am J Surg 1995; 169: 91 - 96

[85] O’Brien PE, Dixon JB. A rational approach to cholelithiasis in bariatric surgery: its application to the laparoscopically placed adjustable gastric band. Arch Surg 2003; 138: 908 -912

[86] Williams C, Gowan R, Perey B]. A double-blind placebo-controlled trial of ursodeoxycholic acid in the prevention of gallstones during weight loss after vertical banded gastroplasty. Obes Surg 1993; 3: 257 - 259

[87] Worobetz LJ, Inglis FG, Shaffer EA. The effect of ursodeoxycholic acid therapy on gallstone formation in the morbidly obese during rapid weight loss. Am J Gastroenterol 1993; 88: 1705-1710

[88] Shiffman ML, Kaplan GD, Brinkman-Kaplan V et al. Prophylaxis against gallstone formation with ursodeoxycholic acid in patients participating in a very-low-calorie diet program. Ann Intern Med 1995; 122: 899-905
[89] Wudel L], Wright JK, Debelak JP et al. Prevention of gallstone formation in morbidly obese patients undergoing rapid weight loss: results of a randomized controlled pilot study. J Surg Res 2002; 102: 50 - 56

[90] Miller K, Hell E, Lang B et al. Gallstone formation prophylaxis after gastric restrictive procedures for weight loss: a randomized double-blind placebo-controlled trial. Ann Surg 2003; 238: 697-702

[91] Villegas L, Schneider B, Provost D et al. Is routine cholecystectomy required during laparoscopic gastric bypass? Obes Surg 2004; 14: 206 211

[92] Ellner S], Myers TT, Piorkowski JR et al. Routine cholecystectomy is not mandatory during morbid obesity surgery. Surg Obes Relat Dis 2007; 3: $456-460$

[93] Rosmorduc O, Hermelin B, Poupon R. MDR3 gene defect in adults with symptomatic intrahepatic and gallbladder cholesterol cholelithiasis. Gastroenterology 2001; 120: 1459-1467

[94] Rosmorduc O, Hermelin B, Boelle PY et al. ABCB4 gene mutation-associated cholelithiasis in adults. Gastroenterology 2003; 125: 452 - 459

[95] Rosmorduc O, Poupon R. Low phospholipid associated cholelithiasis: association with mutation in the MDR3 / ABCB4 gene. Orphanet J Rare Dis 2007; 2: 29

[96] Poupon R, Rosmorduc O, Boelle PY et al. Genotype-phenotype relationships in the low-phospholipid-associated cholelithiasis syndrome: a study of 156 consecutive patients. Hepatology 2013; 58: 1105 - 1110

[97] Arbeitsgemeinschaft der Wissenschaftlichen Medizinischen Fachgesellschaften (AWMF Online). S3-Leitlinie Hormontherapie in der Peri- und Postmenopause (HT). 2009. Registernummer 015-062

[98] Marjoribanks ], Farquhar C, Roberts $\mathrm{H}$ et al. Long-term hormone therapy for perimenopausal and postmenopausal women. Cochrane Database Syst Rev 2017; 1: CD004143

[99] Jørgensen T. Gall stones in a Danish population: fertility period, pregnancies, and exogenous female sex hormones. Gut 1988; 29: 433 - 439

[100] Grodstein F, Colditz GA, Stampfer M]. Postmenopausal hormone use and cholecystectomy in a large prospective study. Obstet Gynecol 1994; 83: 5-11

[101] Cesmeli E, Elewaut AE, Kerre T et al. Gallstone recurrence after successful shock wave therapy: the magnitude of the problem and the predictive factors. Am J Gastroenterol 1999; 94: 474-479

[102] Mamdani MM, Tu K, van Walraven C et al. Postmenopausal estrogen replacement therapy and increased rates of cholecystectomy and appendectomy. CMAJ 2000; 162: 1421 - 1424

[103] La Vecchia C, Negri E, D’Avanzo B et al. Oral contraceptives and noncontraceptive oestrogens in the risk of gallstone disease requiring surgery. J Epidemiol Community Health 1992; 46: 234-236

[104] Schwarz S, Volzke H, Baumeister SE et al. Menopausal hormone therapy and gallbladder disease: the study of health in Pomerania (SHIP). Clin Endocrinol (Oxf) 2007; 67: 51 - 59

[105] Simon JA, Hunninghake DB, Agarwal SK et al. Effect of estrogen plus progestin on risk for biliary tract surgery in postmenopausal women with coronary artery disease. The Heart and Estrogen/progestin Replacement Study. Ann Intern Med 2001; 135: 493 - 501

[106] Rossouw JE, Anderson GL, Prentice RL et al. Risks and benefits of estrogen plus progestin in healthy postmenopausal women: principal results from the Women's Health Initiative randomized controlled trial. JAMA 2002; 288: $321-333$

[107] Cirillo DJ, Wallace RB, Rodabough RJ et al. Effect of estrogen therapy on gallbladder disease. JAMA 2005; 293: 330-339

[108] Hart AR, Luben R, Welch A et al. Hormone replacement therapy and symptomatic gallstones - a prospective population study in the EPICNorfolk cohort. Digestion 2008; 77: 4-9

[109] Liu B, Beral V, Balkwill A et al. Gallbladder disease and use of transdermal versus oral hormone replacement therapy in postmenopausal women: prospective cohort study. BMJ 2008; 337: a386 
[110] Testoni PA, Tittobello A. Long-term efficacy of endoscopic papillosphincterotomy for common bile duct stones and benign papillary stenosis. Surg Endosc 1991; 5: 135-139

[111] Bergman J], van der Mey S, Rauws EA et al. Long-term follow-up after endoscopic sphincterotomy for bile duct stones in patients younger than 60 years of age. Gastrointest Endosc 1996; 44: $643-649$

[112] Lai KH, Peng N], Lo GH et al. Prediction of recurrent choledocholithiasis by quantitative cholescintigrafy in patients after endoscopic sphincterotomy. Gut 1997; 41: 399-403

[113] Kratzer W, Mason RA, Grammer S et al. Difficult bile duct stone recurrence after endoscopy and extracorporeal shockwave lithotripsy. Hepatogastroenterology 1998; 45: 910-916

[114] Tanaka M, Takahata S, Konomi $\mathrm{H}$ et al. Long-term consequence of endoscopic sphincterotomy for bile duct stones. Gastrointest Endosc 1998; 48: 465-469

[115] Sugiyama M, Suzuki Y, Abe $N$ et al. Endoscopic retreatment of recurrent choledocholithiasis after sphincterotomy. Gut 2004; 53: 1856 1859

[116] Lee YN, Moon JH, Choi HJ et al. Direct peroral cholangioscopy using an ultraslim upper endoscope for management of residual stones after mechanical lithotripsy for retained common bile duct stones. Endoscopy 2012; 44: 819-824

[117] von Schonfels W, Buch S, Wolk M et al. Recurrence of gallstones after cholecystectomy is associated with ABCG5/8 genotype. J Gastroenterol 2013; 48: $391-396$

[118] Smit JW, van Erpecum KJ, Renooij W et al. The effects of the 3-hydroxy, 3-methylglutaryl coenzyme A reductase inhibitor pravastatin on bile composition and nucleation of cholesterol crystals in cholesterol gallstone disease. Hepatology 1995; 21: 1523-1529

[119] Galandi D, Schwarzer G, Bassler D et al. Ursodeoxycholic acid and/or antibiotics for prevention of biliary stent occlusion. Cochrane Database Syst Rev 2002; 3: CD003043

[120] Sciume C, Geraci G, Pisello F et al. Prevention of clogging of biliary stents by administration of levofloxacin and ursodeoxycholic acid. Chir Ital 2004; 56: 831 - 837

[121] Chan G, Barkun J, Barkun AN et al. The role of ciprofloxacin in prolonging polyethylene biliary stent patency: a multicenter, double-blinded effectiveness study. J Gastrointest Surg 2005; 9: 481-488

[122] Reuken PA, Torres D, Baier M et al. Risk Factors for Multi-Drug Resistant Pathogens and Failure of Empiric First-Line Therapy in Acute Cholangitis. PLoS One 2017; 12: e0169900

[123] Berger MY, van der Velden J], Lijmer JG et al. Abdominal symptoms: do they predict gallstones? A systematic review. Scand J Gastroenterol 2000; $35: 70-76$

[124] Festi D, Sottili S, Colecchia A et al. Clinical manifestations of gallstone disease: evidence from the multicenter Italian study on cholelithiasis (MICOL). Hepatology 1999; 30: 839-846

[125] Shabanzadeh DM, Sorensen LT, Jorgensen T. Which abdominal symptoms are associated with clinical events in a population unaware of their gallstones? A cohort study. J Gastrointest Surg 2017; 21: 831 839

[126] Berhane T, Vetrhus M, Hausken T et al. Pain attacks in non-complicated and complicated gallstone disease have a characteristic pattern and are accompanied by dyspepsia in most patients: the results of a prospective study. Scand J Gastroenterol 2006; 41: 93-101

[127] Jørgensen T. Abdominal symptoms and gallstone disease: an epidemiological investigation. Hepatology 1989; 9: 856-860

[128] Glambek I, Arnesjo B, Soreide O. Correlation between gallstones and abdominal symptoms in a random population. Results from a screening study. Scand J Gastroenterol 1989; 24: 277-281

[129] Kennedy TM, Jones RH. Epidemiology of cholecystectomy and irritable bowel syndrome in a UK population. Br J Surg 2000; 87: 1658- 1663
[130] Weinert CR, Arnett D, Jacobs D et al. Relationship between persistence of abdominal symptomes and successful outcome after cholecystectomy. Arch Intern Med 2000; 160: 989-995

[131] Thistle JL, Cleary PA, Lachin JM et al. The natural history of cholelithiasis: the National Cooperative Gallstone Study. Ann Intern Med 1984; 101: $171-175$

[132] Friedman GD, Raviola CA, Fireman B. Prognosis of gallstones with mild or no symptoms: 25 years of follow-up in a health maintenance organization. J Clin Epidemiol 1989; 42: 127-136

[133] Ransohoff DF, Gracie WA. Treatment of gallstones. Ann Intern Med 1993; 119: 606-619

[134] Attili AF, De Santis A, Capri R et al. The natural history of gallstones: the GREPCO experience. The GREPCO Group. Hepatology 1995; 21: 655 660

[135] McSherry CK, Ferstenberg H, Calhoun WF et al. The natural history of diagnosed gallstone disease in symptomatic and asymptomatic patients. Ann Surg 1985; 202: 59-63

[136] Gracie WA, Ransohoff DF. The natural history of silent gallstones: the innocent gallstone is not a myth. N Engl J Med 1982; 307: 798-800

[137] Friedman GD. Natural history of asymptomatic and symptomatic gallstones. Am J Surg 1993; 165: 399-404

[138] Festi D, Reggiani ML, Attili AF et al. Natural history of gallstone disease: Expectant management or active treatment? Results from a population-based cohort study. J Gastroenterol Hepatol 2010; 25: 719-724

[139] Shabanzadeh DM, Sorensen LT, Jorgensen T. A prediction rule for risk stratification of incidentally discovered gallstones: Results from a large cohort study. Gastroenterology 2016; 150: 156 - 167.e151

[140] Shea JA, Berlin JA, Escarce J] et al. Revised estimates of diagnostic test sensitivity and specificity in suspected biliary tract disease. Arch Intern Med 1994; 154: $2573-2581$

[141] Ahmed M, Diggory R. The correlation between ultrasonografy and histology in the search for gallstones. Ann R Coll Surg Engl 2011; 93: $81-83$

[142] Mirbagheri SA, Mohamadnejad M, Nasiri J et al. Prospective evaluation of endoscopic ultrasonografy in the diagnosis of biliary microlithiasis in patients with normal transabdominal ultrasonografy. J Gastrointest Surg 2005; 9: $961-964$

[143] Thorbøll J, Vilmann P, Jacobsen B et al. Endoscopic ultrasonografy in detection of cholelithiasis in patients with biliary pain and negative transabdominal ultrasonografy. Scand J Gastroenterol 2004; 39: 267 269

[144] Angelico M, Gandin C, Canuzzi P et al. Gallstones in cystic fibrosis: a critical reappraisal. Hepatology 1991; 14: $768-775$

[145] Fracchia M, Pellegrino S, Secreto P et al. Biliary lipid composition in cholesterol microlithiasis. Gut 2001; 48: 702 - 706

[146] Condat B, Zanditenas D, Barbu V et al. Prevalence of low phospholipidassociated cholelithiasis in young female patients. Dig Liver Dis 2013; 45: $915-919$

[147] Van Mil SW, van der Woerd WL, van der Brugge $G$ et al. Benign recurrent intrahepatic cholestasis type 2 is caused by mutations in ABCB11. Gastroenterology 2004; 127: 379-384

[148] Duggirala R, Mitchell BD, Blangero J et al. Genetic determinants of variation in gallbladder disease in the Mexican-American population. Genet Epidemiol 1999; 16: 191-204

[149] Nakeeb A, Comuzzie AG, Martin L et al. Gallstones: genetics versus environment. Ann Surg 2002; 235: 842-849

[150] Katsika D, Grjibovski A, Einarsson C et al. Genetic and environmental influences on symptomatic gallstone disease: a Swedish study of 43141 twin pairs. Hepatology 2005; 41: $1138-1143$

[151] Puppala S, Dodd GD, Fowler $S$ et al. A genomewide search finds major susceptibility loci for gallbladder disease on chromosome 1 in Mexican Americans. Am J Hum Genet 2006; 78: 377-392 
[152] Buch S, Schafmayer C, Volzke H et al. A genome-wide association scan identifies the hepatic cholesterol transporter ABCG8 as a susceptibility factor for human gallstone disease. Nat Genet 2007; 39: 995-999

[153] Grünhage F, Acalovschi M, Tirziu S et al. Increased gallstone risk in humans conferred by common variant of hepatic ATP-binding cassette transporter for cholesterol. Hepatology 2007; 46: 793-801

[154] Kiewiet J], Leeuwenburgh MM, Bipat S et al. A systematic review and metaanalysis of diagnostic performance of imaging in acute cholecystitis. Radiology 2012; 264: $708-720$

[155] O'Connor OJ, Maher MM. Imaging of cholecystitis. Am J Roentgenol 2011; 196: W367-W374

[156] Yokoe M, Takada T, Strasberg SM et al. New diagnostic criteria and severity assessment of acute cholecystitis in revised Tokyo guidelines. J Hepatobiliary Pancreat Sci 2012; 19: 578- 585

[157] Pinto A, Reginelli A, Cagini L et al. Accuracy of ultrasonografy in the diagnosis of acute calculous cholecystitis: review of the literature. Crit Ultrasound J 2013; 5 (Suppl. 1): S11

[158] Ralls PW, Halls ], Lapin SA et al. Prospective evaluation of the sonografic Murphy sign in suspected acute cholecystitis. J Clin Ultrasound 1982; 10: $113-115$

[159] Cwik G, Skoczylas T, Wyroslak-Najs J et al. The value of percutaneous ultrasound in predicting conversion from laparoscopic to open cholecystectomy due to acute cholecystitis. Surg Endosc 2013; 27: 2561 2568

[160] Altun E, Semelka RC, Elias J Jr et al. Acute cholecystitis: MR findings and differentiation from chronic cholecystitis. Radiology 2007; 244: 174 183

[161] Fuks D, Mouly C, Robert B et al. Acute cholecystitis: preoperative CT can help the surgeon consider conversion from laparoscopic to open cholecystectomy. Radiology 2012; 263: 128-138

[162] Revel L, Lubrano J, Badet $\mathrm{N}$ et al. Preoperative diagnosis of gangrenous acute cholecystitis: usefulness of CEUS. Abdom Imaging 2014; 39 : $1175-1181$

[163] Benarroch-Gampel ], Boyd CA, Sheffield KM et al. Overuse of CT in patients with complicated gallstone disease. J Am Coll Surg 2011; 213: $524-530$

[164] Tonolini M, Ravelli A, Villa C et al. Urgent MRI with MR cholangiopancreatografy (MRCP) of acute cholecystitis and related complications: diagnostic role and spectrum of imaging findings. Emerg Radiol 2012; 19: $341-348$

[165] Soyer P, Hoeffel C, Dohan A et al. Acute cholecystitis: quantitative and qualitative evaluation with 64-section helical CT. Acta Radiol 2013; 54 : $477-486$

[166] Kaoutzanis C, Davies E, Leichtle SW et al. Abdominal ultrasound versus hepato-imino diacetic acid scan in diagnosing acute cholecystitiswhat is the real benefit? J Surg Res 2014; 188: 44-52

[167] Gutt CN, Encke J, Koninger J et al. Acute cholecystitis: early versus delayed cholecystectomy, a multicenter randomized trial (ACDC study, NCT00447304). Ann Surg 2013; 258: 385-393

[168] Summers SM, Scruggs W, Menchine MD et al. A prospective evaluation of emergency department bedside ultrasonografy for the detection of acute cholecystitis. Ann Emerg Med 2010; 56: 114-122

[169] Carroll PJ, Gibson D, El-Faedy O et al. Surgeon-performed ultrasound at the bedside for the detection of appendicitis and gallstones: systematic review and metaanalysis. Am J Surg 2013; 205: $102-108$

[170] Irkorucu O, Reyhan E, Erdem H et al. Accuracy of surgeon-performed gallbladder ultrasound in identification of acute cholecystitis. J Invest Surg 2013; 26: $85-88$

[171] Suk KT, Kim CH, Baik SK et al. Gallbladder wall thickening in patients with acute hepatitis. J Clin Ultrasound 2009; 37: 144-148
[172] Smith EA, Dillman JR, Elsayes KM et al. Cross-sectional imaging of acute and chronic gallbladder inflammatory disease. Am J Roentgenol 2009; 192: $188-196$

[173] Runner G], Corwin MT, Siewert B et al. Gallbladder wall thickening. Am J Roentgenol 2014; 202: W1 - W12

[174] Bharath Kumar Reddy KR, Laksmana RR, Veerappa BG et al. Ultrasonografy as a tool in predicting the severity of dengue fever in children-a useful aid in a developing country. Pediatr Radiol 2013; 43: 971 - 977

[175] Park SJ, Kim JD, Seo YS et al. Computed tomografy findings for predicting severe acute hepatitis with prolonged cholestasis. World J Gastroenterol 2013; 19: 2543 - 2549

[176] Kai K, Ide T, Masuda M et al. Clinicopathologic features of advanced gallbladder cancer associated with adenomyomatosis. Virchows Arch 2011; 459: 573 - 580

[177] Bang SH, Lee JY, Woo H et al. Differentiating between adenomyomatosis and gallbladder cancer: revisiting a comparative study of highresolution ultrasound, multidetector $C T$, and MR imaging. Korean J Radiol 2014; 15: 226-234

[178] Joo I, Lee JY, Kim JH et al. Differentiation of adenomyomatosis of the gallbladder from early-stage, wall-thickening-type gallbladder cancer using high-resolution ultrasound. Eur Radiol 2013; 23: 730-738

[179] Choi JH, Seo DW, Choi JH et al. Utility of contrast-enhanced harmonic EUS in the diagnosis of malignant gallbladder polyps (with videos). Gastrointest Endosc 2013; 78: 484-493

[180] Imazu H, Mori N, Kanazawa K et al. Contrast-enhanced harmonic endoscopic ultrasonografy in the differential diagnosis of gallbladder wall thickening. Dig Dis Sci 2014; 59: 1909 - 1916

[181] Abboud PA, Malet PF, Berlin JA et al. Predictors of common bile duct stones prior to cholecystectomy: a metaanalysis. Gastrointest Endosc 1996; 44: $450-455$

[182] Trondsen E, Edwin B, Reiertsen O et al. Prediction of common bile duct stones prior to cholecystectomy: a prospective validation of a discriminant analysis function. Arch Surg 1998; 133: 162-166

[183] Fahlke J, Ridwelski K, Manger T et al. Diagnostic workup before laparoscopic cholecystectomy - which diagnostic tools should be used? Hepatogastroenterology 2001; 48: 59-65

[184] Lamberts MP, Kievit W, Ozdemir C et al. Value of EGD in patients referred for cholecystectomy: a systematic review and metaanalysis. Gastrointest Endosc 2015; 82: 24-31

[185] Aronson N, Flamm CR, Mark D et al. Endoscopic retrograde cholangiopancreatografy. Evid Rep Technol Assess 2002; 50: 1 - 369

[186] Barkun AN, Barkun JS, Fried GM et al. Useful predictors of bile duct stones in patients undergoing laparoscopic cholecystectomy. McGill gallstone treatment group. Ann Surg 1994; 220: 32 - 39

[187] Koo KP, Traverso LW. Do preoperative indicators predict the presence of common bile duct stones during laparoscopic cholecystectomy? Am J Surg 1996; 171: 495-499

[188] Onken JE, Brazer SR, Eisen GM et al. Predicting the presence of choledocholithiasis in patients with symptomatic cholelithiasis. Am J Gastroenterol 1996; 91: $762-767$

[189] Santucci L, Natalini G, Sarpi L et al. Selective endoscopic retrograde cholangiografy and preoperative bile duct stone removal in patients scheduled for laparoscopic cholecystectomy: a prospective study. Am J Gastroenterol 1996; 91: 1326-1330

[190] Alponat A, Kum CK, Rajnakova A et al. Predictive factors for synchronous common bile duct stones in patients with cholelithiasis. Surg Endosc 1997; 11: $928-932$

[191] Kim KH, Kim W, Lee HI et al. Prediction of common bile duct stones: its validation in laparoscopic cholecystectomy. Hepatogastroenterology 1997; 44: $1574-1579$ 
[192] Bergamaschi R, Tuech J], Braconier L et al. Selective endoscopic retrograde cholangiografy prior to laparoscopic cholecystectomy for gallstones. Am J Surg 1999; 178: 46-49

[193] Masci E, Fanti L, Mariani A et al. Selection criteria for pre-operative endoscopic retrograde cholangiografy and endoscopic-laparoscopic treatment of biliary stones. Eur J Gastroenterol Hepatol 1999; 11: $781-784$

[194] Prat F, Amouyal G, Amouyal P et al. Prospective controlled study of endoscopic ultrasonografy and endoscopic retrograde cholangiografy in patients with suspected common-bileduct lithiasis. Lancet 1996; 347: $75-79$

[195] Sahai AV, Mauldin PD, Marsi V et al. Bile duct stones and laparoscopic cholecystectomy: a decision analysis to assess the roles of intraoperative cholangiografy, EUS, and ERCP. Gastrointest Endosc 1999; 49: 334-343

[196] Kama NA, Atli M, Doganay M et al. Practical recommendations for the prediction and management of common bile duct stones in patients with gallstones. Surg Endosc 2001; 15: 942-945

[197] Liu CL, Lo CM, Chan JK et al. Detection of choledocholithiasis by EUS in acute pancreatitis: a prospective evaluation in 100 consecutive patients. Gastrointest Endosc 2001; 54: 325-330

[198] Luman W, Chan ES. Mathematical model for predicting biliary therapeutic endoscopic retrograde pancreatografy (ERCP). Dig Liver Dis 2003; 35: 486-492

[199] Sarli L, Costi R, Gobbi S et al. Scoring system to predict asymptomatic choledocholithiasis before laparoscopic cholecystectomy. A matched case-control study. Surg Endosc 2003; 17: 1396 - 1403

[200] Grande M, Torquati A, Tucci G et al. Preoperative risk factors for common bile duct stones: defining the patient at high risk in the laparoscopic cholecystectomy era. J Laparoendosc Adv Surg Tech A 2004; 14: $281-286$

[201] Yang MH, Chen TH, Wang SE et al. Biochemical predictors for absence of common bile duct stones in patients undergoing laparoscopic cholecystectomy. Surg Endosc 2008; 22: 1620-1624

[202] Horwood J, Akbar F, Davis K et al. Prospective evaluation of a selective approach to cholangiografy for suspected common bile duct stones. Ann R Coll Surg Engl 2010; 92: 206-210

[203] Jovanovic P, Salkic NN, Zerem E et al. Biochemical and ultrasound parameters may help predict the need for therapeutic endoscopic retrograde cholangiopancreatografy (ERCP) in patients with a firm clinical and biochemical suspicion for choledocholithiasis. Eur ] Intern Med 2011; 22: e110-e114

[204] Trivedi P], Tse D, Al-Bakir I et al. Appropriate patient selection in the management of common bile duct stones: when not to do ERCP. ISRN Surg 2012; 2012: 286365

[205] Al-jiffry BO, Elfateh A, Chundrigar T et al. Non-invasive assessment of choledocholithiasis in patients with gallstones and abnormal liver function. World J Gastroenterol 2013; 19: 5877-5882

[206] Isherwood J, Garcea G, Williams R et al. Serology and ultrasound for diagnosis of choledocholithiasis. Ann R Coll Surg Engl 2014; 96: 224 228

[207] Rickes S, Treiber G, Monkemüller K et al. Impact of the operator's experience on value of high-resolution transabdominal ultrasound in the diagnosis of choledocholithiasis: a prospective comparison using endoscopic retrograde cholangiografy as the gold standard. Scand J Gastroenterol 2006; 41: 838-843

[208] Ripolles T, Ramirez-Fuentes C, Martinez-Perez M] et al. Tissue harmonic sonografy in the diagnosis of common bile duct stones: a comparison with endoscopic retrograde cholangiografy. J Clin Ultrasound 2009; 37: $501-506$

[209] Gurusamy KS, Giljaca V, Takwoingi Y et al. Ultrasound versus liver function tests for diagnosis of common bile duct stones. Cochrane Database Syst Rev 2015; 2: CD011548
[210] Boys JA, Doorly MG, Zehetner ] et al. Can ultrasound common bile duct diameter predict common bile duct stones in the setting of acute cholecystitis? Am J Surg 2014; 207: 432 - 435; discussion 435

[211] Maple JT, Ben-Menachem T, Committee ASoP et al. The role of endoscopy in the evaluation of suspected choledocholithiasis. Gastrointest Endosc 2010; 71: 1-9

[212] Sienz M, Ignee A, Dietrich CF. Reference values in abdominal ultrasound - biliopancreatic system and spleen. Z Gastroenterol 2011; 49: $845-870$

[213] Daradkeh S, Tarawneh E, Al-Hadidy A. Factors affecting common bile duct diameter. Hepatogastroenterology 2005; 52: 1659-1661

[214] Benjaminov F, Leichtman G, Naftali T et al. Effects of age and cholecystectomy on common bile duct diameter as measured by endoscopic ultrasonografy. Surg Endosc 2013; 27: 303-307

[215] McArthur TA, Planz V, Fineberg NS et al. The common duct dilates after cholecystectomy and with advancing age: reality or myth? J Ultrasound Med 2013; 32: 1385-1391

[216] van Santvoort HC, Bakker OJ, Besselink MG et al. Prediction of common bile duct stones in the earliest stages of acute biliary pancreatitis. Endoscopy 2011; 43: 8-13

[217] Costi R, Sarli L, Caruso G et al. Preoperative ultrasonografic assessment of the number and size of gallbladder stones: is it a useful predictor of asymptomatic choledochal lithiasis? J Ultrasound Med 2002; 21: 971 976

[218] Huguier M, Bornet P, Charpak Y et al. Selective contraindications based on multivariate analysis for operative cholangiografy in biliary lithiasis. Surg Gynecol Obstet 1991; 172: 470-474

[219] Cohen S, Bacon BR, Berlin JA et al. NIH state-of-the-science statement on endoscopic retrograde cholangiopancreatografy (ERCP) for diagnosis and therapy. NIH Consensus State Sci Statements 2002; 19: 1 26

[220] Chhoda A, Jain D, Singhal S. Sex-related differences in predicting choledocholithiasis using current American Society of Gastrointestinal Endoscopy risk criteria. Ann Gastroenterol 2017; 30: 682 -687

[221] Topal B, Fieuws S, Tomczyk K et al. Clinical models are inaccurate in predicting bile duct stones in situ for patients with gallbladder. Surg Endosc 2009; 23: 38 - 44

[222] Sugiyama M, Atomi Y. Acute biliary pancreatitis: the roles of endoscopic ultrasonografy and endoscopic retrograde cholangiopancreatografy. Surgery 1998; 124: 14-21

[223] Chak A, Hawes RH, Cooper GS et al. Prospective assessment of the utility of EUS in the evaluation of gallstone pancreatitis. Gastrointest Endosc 1999; 49: 599-604

[224] Palazzo L, Girollet PP, Salmeron M et al. Value of endoscopic ultrasonografy in the diagnosis of common bile duct stones: comparison with surgical exploration and ERCP. Gastrointest Endosc 1995; 42: 225 231

[225] Tseng LJ, Jao YT, Mo LR et al. Over-the-wire US catheter probe as an adjunct to ERCP in the detection of choledocholithiasis. Gastrointest Endosc 2001; 54: 720-723

[226] Moon JH, Cho YD, Cha SW et al. The detection of bile duct stones in suspected biliary pancreatitis: comparison of MRCP, ERCP, and intraductal US. Am J Gastroenterol 2005; 100: 1051 - 1057

[227] Freeman ML. Complications of endoscopic retrograde cholangiopancreatografy: avoidance and management. Gastrointest Endosc Clin N Am 2012; 22: 567-586

[228] Johnson AG, Hosking SW. Appraisal of the management of bile duct stones. Br J Surg 1987; 74: 555-560

[229] Murison MS, Gartell PC, McGinn FP. Does selective peroperative cholangiografy result in missed common bile duct stones? J R Coll Surg Edinb 1993; 38: 220 - 224 
[230] Arguedas MR, Dupont AW, Wilcox CM. Where do ERCP, endoscopic ultrasound, magnetic resonance cholangiopancreatografy, and intraoperative cholangiografy fit in the management of acute biliary pancreatitis? A decision analysis model. Am J Gastroenterol 2001; 96 : $2892-2899$

[231] Scheiman JM, Carlos RC, Barnett JL et al. Can endoscopic ultrasound or magnetic resonance cholangiopancreatografy replace ERCP in patients with suspected biliary disease? A prospective trial and cost analysis. Am J Gastroenterol 2001; 96: 2900-2904

[232] Palazzo L, O'Toole D. EUS in common bile duct stones. Gastrointest Endosc 2002; 56: S49-S57

[233] Wang B, Guo Z, Liu Z et al. Preoperative versus intraoperative endoscopic sphincterotomy in patients with gallbladder and suspected common bile duct stones: system review and metaanalysis. Surg Endosc 2013; 27: 2454-2465

[234] Gurusamy K, Sahay S], Burroughs AK et al. Systematic review and metaanalysis of intraoperative versus preoperative endoscopic sphincterotomy in patients with gallbladder and suspected common bile duct stones. Br J Surg 2011; 98: 908 -916

[235] Prachayakul V, Aswakul P, Bhunthumkomol P et al. Diagnostic yield of endoscopic ultrasonografy in patients with intermediate or high likelihood of choledocholithiasis: a retrospective study from one universitybased endoscopy center. BMC Gastroenterol 2014; 14: 165

[236] $\mathrm{He} \mathrm{H}$, Tan C, Wu J et al. Accuracy of ASGE high-risk criteria in evaluation of patients with suspected common bile duct stones. Gastrointest Endosc 2017; 86: 525 - 532

[237] Sethi S, Wang F, Korson AS et al. Prospective assessment of consensus criteria for evaluation of patients with suspected choledocholithiasis. Dig Endosc 2016; 28: 75 - 82

[238] Suarez AL, LaBarre NT, Cotton PB et al. An assessment of existing risk stratification guidelines for the evaluation of patients with suspected choledocholithiasis. Surg Endosc 2016; 30: 4613-4618

[239] Adams MA, Hosmer AE, Wamsteker EJ et al. Predicting the likelihood of a persistent bile duct stone in patients with suspected choledocholithiasis: accuracy of existing guidelines and the impact of laboratory trends. Gastrointest Endosc 2015; 82: 88 -93

[240] Narvaez Rivera RM, Gonzalez Gonzalez JA, Monreal Robles R et al. Accuracy of ASGE criteria for the prediction of choledocholithiasis. Rev Esp Enferm Dig 2016; 108: 309-314

[241] Patel R, Ingle M, Choksi D et al. Endoscopic ultrasonografy can prevent unnecessary diagnostic endoscopic retrograde cholangiopancreatografy even in patients with high likelihood of choledocholithiasis and inconclusive ultrasonografy: results of a prospective study. Clin Endosc 2017; 50: $592-597$

[242] Kuzu UB, Odemis B, Disibeyaz S et al. Management of suspected common bile duct stone: diagnostic yield of current guidelines. HPB (Oxford) 2017; 19: 126-132

[243] Rubin MI, Thosani NC, Tanikella R et al. Endoscopic retrograde cholangiopancreatografy for suspected choledocholithiasis: testing the current guidelines. Dig Liver Dis 2013; 45: 744-749

[244] Ainsworth AP, Rafaelsen SR, Wamberg PA et al. Is there a difference in diagnostic accuracy and clinical impact between endoscopic ultrasonografy and magnetic resonance cholangiopancreatografy? Endoscopy 2003; 35: 1029-1032

[245] Fusaroli P, Lisotti A, Syguda A et al. Reliability of endoscopic ultrasound in predicting the number and size of common bile duct stones before endoscopic retrograde cholangiopancreatografy. Dig Liver Dis 2016; 48: $277-282$

[246] Garrow D, Miller S, Sinha D et al. Endoscopic ultrasound: a metaanalysis of test performance in suspected biliary obstruction. Clin Gastroenterol Hepatol 2007; 5: 616-623

[247] Polkowski M, Regula J, Tilszer A et al. Endoscopic ultrasound versus endoscopic retrograde cholangiografy for patients with intermediate probability of bile duct stones: a randomized trial comparing two management strategies. Endoscopy 2007; 39: 296-303

[248] Lee YT, Chan FK, Leung WK et al. Comparison of EUS and ERCP in the investigation with suspected biliary obstruction caused by choledocholithiasis: a randomized study. Gastrointest Endosc 2008; 67: 660 668

[249] Karakan T, Cindoruk M, Alagozlu $\mathrm{H}$ et al. EUS versus endoscopic retrograde cholangiografy for patients with intermediate probability of bile duct stones: a prospective randomized trial. Gastrointest Endosc 2009; 69: $244-252$

[250] Sharma R, Menachery ], Choudhary NS et al. Routine endoscopic ultrasound in moderate and indeterminate risk patients of suspected choledocholithiasis to avoid unwarranted ERCP: A prospective randomized blinded study. Indian J Gastroenterol 2015; 34: 300 - 304

[251] Jenssen C, Alvarez-Sanchez MV, Napoleon B et al. Diagnostic endoscopic ultrasonografy: assessment of safety and prevention of complications. World J Gastroenterol 2012; 18: 4659-4676

[252] Sugiyama M, Atomi Y. Endoscopic ultrasonografy for diagnosing choledocholithiasis: a prospective comparative study with ultrasonografy and computed tomografy. Gastrointest Endosc 1997; 45: 143-146

[253] Dancygier H, Nattermann C. The role of endoscopic ultrasonografy in biliary tract disease: obstructive jaundice. Endoscopy 1994; 26: 800 802

[254] Norton SA, Alderson D. Prospective comparison of endoscopic ultrasonografy and endoscopic retrograde cholangiopancreatografy in the detection of bile duct stones. Br J Surg 1997; 84: 1366-1369

[255] Canto MI, Chak A, Stellato T et al. Endoscopic ultrasonografy versus cholangiografy for the diagnosis of choledocholithiasis. Gastrointest Endosc 1998; 47: 439-448

[256] De Ledinghen V, Lecesne R, Raymond JM et al. Diagnosis of choledocholithiasis: EUS or magnetic resonance cholangiografy? A prospective controlled study. Gastrointest Endosc 1999; 49: 26-31

[257] Polkowski M, Palucki J, Regula J et al. Helical computed tomografic cholangiografy versus endosonografy for suspected bile duct stones: a prospective blinded study in non-jaundiced patients. Gut 1999; 45: $744-749$

[258] Materne R, van Beers BE, Gigot JF et al. Extrahepatic biliary obstruction: magnetic resonance imaging compared with endoscopic ultrasonografy. Endoscopy 2000; 32: 3-9

[259] Buscarini E, Tansini P, Vallisa D et al. EUS for suspected choledocholithiasis: do benefits outweigh costs? A prospective, controlled study. Gastrointest Endosc 2003; 57: 510 - 518

[260] Napoleon B, Dumortier ], Keriven-Souquet O et al. Do normal findings at biliary endoscopic ultrasonografy obviate the need for endoscopic retrograde cholangiografy in patients with suspicion of common bile duct stone? A prospective follow-up study of 238 patients. Endoscopy 2003; 35: $411-415$

[261] Tse F, Barkun JS, Barkun AN. The elective evaluation of patients with suspected choledocholithiasis undergoing laparoscopic cholecystectomy. Gastrointest Endosc 2004; 60: 437 - 448

[262] Aubé C, Delorme B, Yzet T et al. MR cholangiopancreatografy versus endoscopic sonografy in suspected common bile duct lithiasis: a prospective, comparative study. Am J Roentgenol 2005; 184: 55 - 62

[263] Kondo S, Isayama H, Akahane M et al. Detection of common bile duct stones: comparison between endoscopic ultrasonografy, magnetic resonance cholangiografy, and helical-computed-tomografic cholangiografy. Eur J Radiol 2005; 54: 271 - 275

[264] Ney MV, Maluf-Filho F, Sakai P et al. Echo-endoscopy versus endoscopic retrograde cholangiografy for the diagnosis of choledocholithiasis: the influence of the size of the stone and diameter of the common bile duct. Arq Gastroenterol 2005; 42: 239-243

[265] Ang TL, Teo EK, Fock KM. Endosonografy- vs. endoscopic retrograde cholangiopancreatografy-based strategies in the evaluation of sus- 
pected common bile duct stones in patients with normal transabdominal imaging. Aliment Pharmacol Ther 2007; 26: 1163-1170

[266] Aljebreen A, Azzam N, Eloubeidi MA. Prospective study of endoscopic ultrasound performance in suspected choledocholithiasis. J Gastroenterol Hepatol 2008; 23: $741-745$

[267] Jeon TJ, Cho JH, Kim YS et al. Diagnostic value of endoscopic ultrasonografy in symptomatic patients with high and intermediate probabilities of common bile duct stones and a negative computed tomografy scan. Gut Liver 2017; 11: $290-297$

[268] Giljaca V, Gurusamy KS, Takwoingi Y et al. Endoscopic ultrasound versus magnetic resonance cholangiopancreatografy for common bile duct stones. Cochrane Database Syst Rev 2015; 2: CD011549

[269] Tse F, Liu L, Barkun AN et al. EUS: a metaanalysis of test performance in suspected choledocholithiasis. Gastrointest Endosc 2008; 67: 235 244

[270] Lachter ], Rubin A, Shiller M et al. Linear EUS for bile duct stones. Gastrointest Endosc 2000; 51: 51 - 54

[271] Kohut M, Nowakowska-Dulawa E, Marek T et al. Accuracy of linear endoscopic ultrasonografy in the evaluation of patients with suspected common bile duct stones. Endoscopy 2002; 34: 299-303

[272] Kohut M, Nowak A, Nowakowska-Dulawa E et al. Endosonografy with linear array instead of endoscopic retrograde cholangiografy as the diagnostic tool in patients with moderate suspicion of common bile duct stones. World J Gastroenterol 2003; 9: 612-614

[273] Janssen J, Halboos A, Greiner L. EUS accurately predicts the need for therapeutic ERCP in patients with a low probability of biliary obstruction. Gastrointest Endosc 2008; 68: 470-476

[274] Wehrmann T, Martchenko K, Riphaus A. Catheter probe extraductal ultrasonografy vs. conventional endoscopic ultrasonografy for detection of bile duct stones. Endoscopy 2009; 41: 133-137

[275] Chan HH, Wang EM, Sun MS et al. Linear echoendoscope-guided ERCP for the diagnosis of occult common bile duct stones. BMC Gastroenterol 2013; 13: 44

[276] Wang M, He X, Tian C et al. The diagnostic accuracy of linear endoscopic ultrasound for evaluating symptoms suggestive of common bile duct stones. Gastroenterol Res Pract 2016; 2016: 6957235

[277] Bang BW, Hong JT, Choi YC et al. Is endoscopic ultrasound needed as an add-on test for gallstone diseases without choledocholithiasis on multidetector computed tomografy? Dig Dis Sci 2012; 57: 3246 3251

[278] Varghese JC, Liddell RP, Farrell MA et al. The diagnostic accuracy of magnetic resonance cholangiopancreatografy and ultrasound compared with direct cholangiografy in the detection of choledocholithiasis. Clin Radiol 1999; 54: 604-614

[279] Guibaud L, Bret PM, Reinhold C et al. Bile duct obstruction and choledocholithiasis: diagnosis with MR cholangiografy. Radiology 1995; 197: $109-115$

[280] Chan YL, Chan AC, Lam WW et al. Choledocholithiasis: comparison of MR cholangiografy and endoscopic retrograde cholangiografy. Radiology 1996; 200: 85-89

[281] Reuther G, Kiefer B, Tuchmann A et al. MR-Cholangiopankreatikografie als Einzelschussprojektion: Erfahrungen und Ergebnisse bei 200 Untersuchungen. Röfo 1996; 165: 535-543

[282] Pavone P, Laghi A, Lomanto D et al. MR cholangiografy (MRC) in the evaluation of CBD stones before laparoscopic cholecystectomy. Surg Endosc 1997; 11: $982-985$

[283] Adamek HE, Albert ], Weitz M et al. A prospective evaluation of magnetic resonance cholangiopancreatografy in patients with suspected bile duct obstruction. Gut 1998; 43: 680-683

[284] Holzknecht N, Gauger J, Sackmann M et al. Breath-hold MR cholangiografy with snapshot techniques: prospective comparison with endoscopic retrograde cholangiografy. Radiology 1998; 206: 657-664
[285] Lomas D], Bearcroft PW, Gimson AE. MR cholangiopancreatografy: prospective comparison of a breath-hold 2D projection technique with diagnostic ERCP. Eur Radiol 1999; 9: 1411-1417

[286] Zidi SH, Prat F, Le Guen O et al. Use of magnetic resonance cholangiografy in the diagnosis of choledocholithiasis: prospective comparison with a reference imaging method. Gut 1999; 44: 118-122

[287] Angulo P, Pearce DH, Johnson CD et al. Magnetic resonance cholangiografy in patients with biliary disease: its role in primary sclerosing cholangitis. J Hepatol 2000; 33: 520-527

[288] Demartines N, Eisner L, Schnabel K et al. Evaluation of magnetic resonance cholangiografy in the management of bile duct stones. Arch Surg 2000; 135: 148-152

[289] Soto JA, Alvarez O, Munera F et al. Diagnosing bile duct stones: comparison of unenhanced helical CT, oral contrast-enhanced CT cholangiografy, and MR cholangiografy. Am J Roentgenol 2000; 175: 1127 1134

[290] Soto JA, Barish MA, Alvarez O et al. Detection of choledocholithiasis with MR cholangiografy: comparison of three-dimensional fast spinecho and single- and multisection half-Fourier rapid acquisition with relaxation enhancement sequences. Radiology 2000; 215: 737 - 745

[291] Stiris MG, Tennoe B, Aadland E et al. MR cholangiopancreaticografy and endoscopic retrograde cholangiopancreaticografy in patients with suspected common bile duct stones. Acta Radiol 2000; 41: 269-272

[292] Laokpessi A, Bouillet P, Sautereau D et al. Value of magnetic resonance cholangiografy in the preoperative diagnosis of common bile duct stones. Am J Gastroenterol 2001; 96: 2354-2359

[293] Boraschi P, Gigoni R, Braccini G et al. Detection of common bile duct stones before laparoscopic cholecystectomy. Evaluation with MR cholangiografy. Acta Radiol 2002; 43: 593 - 598

[294] Calvo MM, Bujanda L, Calderon A et al. Role of magnetic resonance cholangiopancreatografy in patients with suspected choledocholithiasis. Mayo Clin Proc 2002; 77: 422 - 428

[295] Hakansson K, Ekberg O, Hakansson HO et al. MR and ultrasound in screening of patients with suspected biliary tract disease. Acta Radiol 2002; 43: $80-86$

[296] Hussein FM, Alsumait B, Aman S et al. Diagnosis of choledocholithiasis and bile duct stenosis by magnetic resonance cholangiogram. Australas Radiol 2002; 46: $41-46$

[297] Jendresen MB, Thorboll JE, Adamsen S et al. Preoperative routine magnetic resonance cholangiopancreatografy before laparoscopic cholecystectomy: a prospective study. Eur J Surg 2002; 168: 690-694

[298] Kim TK, Kim BS, Kim JH et al. Diagnosis of intrahepatic stones: superiority of MR cholangiopancreatografy over endoscopic retrograde cholangiopancreatografy. Am J Roentgenol 2002; 179: 429-434

[299] Kim JH, Kim MJ, Park SI et al. MR cholangiografy in symptomatic gallstones: diagnostic accuracy according to clinical risk group. Radiology 2002; 224: $410-416$

[300] Taylor AC, Little AF, Hennessy OF et al. Prospective assessment of magnetic resonance cholangiopancreatografy for noninvasive imaging of the biliary tree. Gastrointest Endosc 2002; 55: 17-22

[301] Urban M, Holzer B, Sebesta C et al. Efficacy of diagnosis of mechanical cholestasis by magnetic resonance cholangiografy. World J Surg 2002; 26: $353-358$

[302] Griffin N, Wastle ML, Dunn WK et al. Magnetic resonance cholangiopancreatografy versus endoscopic retrograde cholangiopancreatografy in the diagnosis of choledocholithiasis. Eur ] Gastroenterol Hepatol 2003; 15: $809-813$

[303] Kats J, Kraai M, Dijkstra AJ et al. Magnetic resonance cholangiopancreaticografy as a diagnostic tool for common bile duct stones: a comparison with ERCP and clinical follow-up. Dig Surg 2003; 20: 32 37 
[304] Romagnuolo J, Bardou M, Rahme E et al. Magnetic resonance cholangiopancreatografy: a metaanalysis of test performance in suspected biliary disease. Ann Intern Med 2003; 139: 547 - 557

[305] Kaltenthaler E, Vergel YB, Chilcott J et al. A systematic review and economic evaluation of magnetic resonance cholangiopancreatografy compared with diagnostic endoscopic retrograde cholangiopancreatografy. Health Technol Assess 2004; 8: 1-89

[306] Verma D, Kapedia A, Eisen GM et al. EUS vs MRCP for detection of choledocholithiasis. Gastrointest Endosc 2006; 64: 248-254

[307] Shanmugam V, Beattie GC, Yule SR et al. Is magnetic resonance cholangiopancreatografy the new gold standard in biliary imaging? $\mathrm{Br}$ ] Radiol 2005; 78: 888-893

[308] Polistina FA, Frego M, Bisello M et al. Accuracy of magnetic resonance cholangiografy compared to operative endoscopy in detecting biliary stones, a single center experience and review of literature. World J Radiol 2015; 7: 70-78

[309] De Castro VL, Moura EG, Chaves DM et al. Endoscopic ultrasound versus magnetic resonance cholangiopancreatografy in suspected choledocholithiasis: A systematic review. Endosc Ultrasound 2016; 5: 118 128

[310] Chen W, Mo JJ, Lin L et al. Diagnostic value of magnetic resonance cholangiopancreatografy in choledocholithiasis. World J Gastroenterol 2015; 21: 3351-3360

[311] Bhat M, Romagnuolo J, da Silveira E et al. Randomised clinical trial: MRCP-first vs. ERCP-first approach in patients with suspected biliary obstruction due to bile duct stones. Aliment Pharmacol Ther 2013; 38: $1045-1053$

[312] Ward WH, Fluke LM, Hoagland BD et al. The role of magnetic resonance cholangiopancreatografy in the diagnosis of choledocholithiasis: do benefits outweigh the costs? Am Surg 2015; 81: 720-725

[313] Meeralam Y, Al-Shammari K, Yaghoobi M. Diagnostic accuracy of EUS compared with MRCP in detecting choledocholithiasis: a metaanalysis of diagnostic test accuracy in head-to-head studies. Gastrointest Endosc 2017; 86: 986-993

[314] Neitlich JD, Topazian M, Smith RC et al. Detection of choledocholithiasis: comparison of unenhanced helical $C T$ and endoscopic retrograde cholangiopancreatografy. Radiology 1997; 203: 753-757

[315] Tseng CW, Chen CC, Chen TS et al. Can computed tomografy with coronal reconstruction improve the diagnosis of choledocholithiasis? J Gastroenterol Hepatol 2008; 23: 1586-1589

[316] Sleytr UB, Umrath W. Proceedings: Double-replicating method for suspensions and tissue fragments. Arzneimittelforschung 1975; 25 : 455

[317] Velanovich V, Morton JM, McDonald M et al. Analysis of the SAGES outcomes initiative cholecystectomy registry. Surg Endosc 2006; 20: 43-50

[318] Varadarajulu S, Eloubeidi MA, Wilcox CM et al. Do all patients with abnormal intraoperative cholangiogram merit endoscopic retrograde cholangiopancreatografy? Surg Endosc 2006; 20: 801-805

[319] Khan OA, Balaji S, Branagan G et al. Randomized clinical trial of routine on-table cholangiografy during laparoscopic cholecystectomy. $\mathrm{Br}$ J Surg 2011; 98: 362 -367

[320] Aziz O, Ashrafian $\mathrm{H}$, Jones $\mathrm{C}$ et al. Laparoscopic ultrasonografy versus intra-operative cholangiogram for the detection of common bile duct stones during laparoscopic cholecystectomy: a metaanalysis of diagnostic accuracy. Int J Surg 2014; 12: 712-719

[321] Hüttl TP, Hrdina C, Krämling H] et al. Gallstone surgery in German university hospitals. Development, complications and changing strategies. Langenbecks Arch Surg 2001; 386: 410-417

[322] Ludwig K, Köckerling F, Hohenberger W et al. Die chirurgische Therapie der Cholecysto-/Choledocholithiasis. Ergebnisse einer deutschlandweiten Umfrage an 859 Kliniken mit 123090 Cholecystektomien. Chirurg 2001; 72: 1171-1178
[323] Ludwig K, Bernhardt J, Lorenz D. Value and consequences of routine intraoperative cholangiografy during cholecystectomy. Surg Laparosc Endosc Percutan Tech 2002; 12: 154-159

[324] Ludwig K, Wilhelm L, Prinz C et al. Die intraoperative laparoskopische Cholangiografie - Wann ist sie sinnvoll? Zentralbl Chir 2004; 129: $185-190$

[325] Niederau C, Muller J, Sonnenberg A et al. Extrahepatic bile ducts in healthy subjects, in patients with cholelithiasis, and in postcholecystectomy patients: a prospective ultrasonic study. J Clin Ultrasound 1983; 11: 23-27

[326] Chawla S, Trick WE, Gilkey S et al. Does cholecystectomy status influence the common bile duct diameter? A matched-pair analysis. Dig Dis Sci 2010; 55: $1155-1160$

[327] Valkovic P, Miletic D, Zelic M et al. Dynamic changes in the common bile duct after laparoscopic cholecystectomy: a prospective longitudinal sonografic study. Ultraschall in Med 2011; 32: 479-484

[328] Park SM, Kim WS, Bae IH et al. Common bile duct dilatation after cholecystectomy: a one-year prospective study. J Korean Surg Soc 2012; 83: $97-101$

[329] Terhaar OA, Abbas S, Thornton FJ et al. Imaging patients with "postcholecystectomy syndrome”: an algorithmic approach. Clin Radiol 2005; 60: 78-84

[330] Howard K, Lord SJ, Speer A et al. Value of magnetic resonance cholangiopancreatografy in the diagnosis of biliary abnormalities in postcholecystectomy patients: a probabilistic cost-effectiveness analysis of diagnostic strategies. Int J Technol Assess Health Care 2006; 22: 109 118

[331] Filip M, Saftoiu A, Popescu C et al. Postcholecystectomy syndrome - an algorithmic approach. J Gastrointestin Liver Dis 2009; 18: 67 - 71

[332] Mohamadnejad M, Hashemi SJ, Zamani F et al. Utility of endoscopic ultrasound to diagnose remnant stones in symptomatic patients after cholecystectomy. Endoscopy 2014; 46: 650-655

[333] Kimura Y, Takada T, Strasberg SM et al. TG13 current terminology, etiology, and epidemiology of acute cholangitis and cholecystitis. J Hepatobiliary Pancreat Sci 2013; 20: 8-23

[334] Rahman SH, Larvin M, McMahon MJ et al. Clinical presentation and delayed treatment of cholangitis in older people. Dig Dis Sci 2005; 50 : $2207-2210$

[335] Rosing DK, De Virgilio C, Nguyen AT et al. Cholangitis: analysis of admission prognostic indicators and outcomes. Am Surg 2007; 73: $949-954$

[336] Kiriyama S, Takada T, Strasberg SM et al. TG13 guidelines for diagnosis and severity grading of acute cholangitis (with videos). J Hepatobiliary Pancreat Sci 2013; 20: 24-34

[337] Sun G, Han L, Yang Y et al. Comparison of two editions of Tokyo guidelines for the management of acute cholangitis. J Hepatobiliary Pancreat Sci 2014; 21: 113-119

[338] Sun G, Han L, Yang YS et al. Verification of the Tokyo guidelines for acute cholangitis secondary to benign and malignant biliary obstruction: experience from a Chinese tertiary hospital. Hepatobiliary Pancreat Dis Int 2013; 12: $400-407$

[339] Kiriyama S, Kozaka K, Takada T et al. Tokyo guidelines 2018: diagnostic and severity grading criteria for acute cholangitis. J Hepatobiliary Pancreat Sci 2018; 25: $17-30$

[340] Catalano OA, Sahani DV, Forcione DG et al. Biliary infections: spectrum of imaging findings and management. Radiografics 2009; 29: 20592080

[341] Miura F, Takada T, Strasberg SM et al. TG13 flowchart for the management of acute cholangitis and cholecystitis. J Hepatobiliary Pancreat Sci 2013; 20: 47-54 
[342] Miura F, Okamoto K, Takada T et al. Tokyo guidelines 2018: initial management of acute biliary infection and flowchart for acute cholangitis. J Hepatobiliary Pancreat Sci 2018; 25: 31-40

[343] Rosen CL, Brown DF, Chang Y et al. Ultrasonografy by emergency physicians in patients with suspected cholecystitis. Am J Emerg Med 2001; 19: $32-36$

[344] Kendall JL, Shimp RJ. Performance and interpretation of focused right upper quadrant ultrasound by emergency physicians. J Emerg Med 2001; 21: 7-13

[345] Balthazar E], Birnbaum BA, Naidich M. Acute cholangitis: CT evaluation. J Comput Assist Tomogr 1993; 17: 283-289

[346] Arai K, Kawai K, Kohda W et al. Dynamic CT of acute cholangitis: early inhomogeneous enhancement of the liver. Am J Roentgenol 2003; 181: $115-118$

[347] Kim SW, Shin HC, Kim HC et al. Diagnostic performance of multidetector $\mathrm{CT}$ for acute cholangitis: evaluation of a CT scoring method. $\mathrm{Br}$ J Radiol 2012; 85: 770-777

[348] Hong M], Kim SW, Kim HC et al. Comparison of the clinical characteristics and imaging findings of acute cholangitis with and without biliary dilatation. Br J Radiol 2012; 85: e1219-e1225

[349] Akaike G, Ishiyama M, Suzuki S et al. Significance of peribiliary oedema on computed tomografy in diagnosis and severity assessment of acute cholangitis. Eur J Radiol 2013; 82: e429-e433

[350] Gaines P, Markham N, Leung J et al. The thick common bile duct in pyogenic cholangitis. Clin Radiol 1991; 44: 175-177

[351] Alper E, Unsal B, Buyrac $Z$ et al. Role of radial endosonografy in the diagnosis of acute cholangitis. Dig Dis Sci 2011; 56: 2191-2196

[352] Eun HW, Kim JH, Hong SS et al. Assessment of acute cholangitis by MR imaging. Eur J Radiol 2012; 81: 2476-2480

[353] Khashab MA, Tariq A, Tariq U et al. Delayed and unsuccessful endoscopic retrograde cholangiopancreatografy are associated with worse outcomes in patients with acute cholangitis. Clin Gastroenterol Hepatol 2012; 10: 1157-1161

[354] Navaneethan U, Gutierrez NG, Jegadeesan R et al. Factors predicting adverse short-term outcomes in patients with acute cholangitis undergoing ERCP: A single center experience. World J Gastrointest Endosc 2014; 6: 74-81

[355] Gigot JF, Leese T, Dereme T et al. Acute cholangitis. Multivariate analysis of risk factors. Ann Surg 1989; 209: 435-438

[356] Lee CC, Chang IJ, Lai YC et al. Epidemiology and prognostic determinants of patients with bacteremic cholecystitis or cholangitis. Am J Gastroenterol 2007; 102: 563-569

[357] Salek J, Livote E, Sideridis K et al. Analysis of risk factors predictive of early mortality and urgent ERCP in acute cholangitis. J Clin Gastroenterol 2009; 43: $171-175$

[358] Lee BS, Hwang JH, Lee SH et al. Risk factors of organ failure in patients with bacteremic cholangitis. Dig Dis Sci 2013; 58: 1091 - 1099

[359] Navaneethan U, Gutierrez NG, Jegadeesan R et al. Delay in performing ERCP and adverse events increase the 30-day readmission risk in patients with acute cholangitis. Gastrointest Endosc 2013; 78: 81-90

[360] Lankisch PG, Assmus C, Maisonneuve P et al. Epidemiology of pancreatic diseases in Luneburg County. A study in a defined German population. Pancreatology 2002; 2: 469-477

[361] Yadav D, Lowenfels AB. Trends in the epidemiology of the first attack of acute pancreatitis: a systematic review. Pancreas 2006; 33: 323 330

[362] Frey CF, Zhou H, Harvey D] et al. The incidence and case-fatality rates of acute biliary, alcoholic, and idiopathic pancreatitis in California, 1994-2001. Pancreas 2006; 33: 336 - 344

[363] van Geenen EJ, van der Peet DL, Bhagirath P et al. Etiology and diagnosis of acute biliary pancreatitis. Nat Rev Gastroenterol Hepatol 2010; 7: $495-502$
[364] Chen Y, Zak Y, Hernandez-Boussard T et al. The epidemiology of idiopathic acute pancreatitis, analysis of the nationwide inpatient sample from 1998 to 2007. Pancreas 2013; 42: 1-5

[365] Venneman NG, Buskens E, Besselink MG et al. Small gallstones are associated with increased risk of acute pancreatitis: potential benefits of prophylactic cholecystectomy? Am J Gastroenterol 2005; 100: $2540-2550$

[366] Davidson BR, Neoptolemos JP, Leese T et al. Biochemical prediction of gallstones in acute pancreatitis: a prospective study of three systems. Br J Surg 1988; 75: 213-215

[367] Mayer AD, McMahon MJ. Biochemical identification of patients with gallstones associated with acute pancreatitis on the day of admission to hospital. Ann Surg 1985; 201: 68-75

[368] Wang SS, Lin XZ, Tsai YT et al. Clinical significance of ultrasonografy, computed tomografy, and biochemical tests in the rapid diagnosis of gallstone-related pancreatitis: a prospective study. Pancreas 1988; 3: $153-158$

[369] Neoptolemos JP, Hall AW, Finlay DF et al. The urgent diagnosis of gallstones in acute pancreatitis: a prospective study of three methods. $\mathrm{Br}$ J Surg 1984; 71: 230-233

[370] Blamey SL, Osborne DH, Gilmour WH et al. The early identification of patients with gallstone associated pancreatitis using clinical and biochemical factors only. Ann Surg 1983; 198: 574-578

[371] Van Gossum A, Seferian V, Rodzynek J] et al. Early detection of biliary pancreatitis. Dig Dis Sci 1984; 29: 97-101

[372] Kazmierczak SC, Catrou PG, Van Lente F. Enzymatic markers of gallstone-induced pancreatitis identified by ROC curve analysis, discriminant analysis, logistic regression, likelihood ratios, and information theory. Clin Chem 1995; 41: 523-531

[373] Tenner S, Dubner H, Steinberg W. Predicting gallstone pancreatitis with laboratory parameters: a metaanalysis. Am J Gastroenterol 1994; 89: $1863-1866$

[374] Chang L, Lo SK, Stabile BE et al. Gallstone pancreatitis: a prospective study on the incidence of cholangitis and clinical predictors of retained common bile duct stones. Am J Gastroenterol 1998; 93: 527 - 531

[375] Pezzilli R, Billi P, Barakat B et al. Ultrasonografic evaluation of the common bile duct in biliary acute pancreatitis patients: comparison with endoscopic retrograde cholangiopancreatografy. J Ultrasound Med 1999; 18: 391 - 394

[376] Ammori B], Boreham B, Lewis P et al. The biochemical detection of biliary etiology of acute pancreatitis on admission: a revisit in the modern era of biliary imaging. Pancreas 2003; 26: e32-e35

[377] Lévy P, Boruchowicz A, Hastier P et al. Diagnostic criteria in predicting a biliary origin of acute pancreatitis in the era of endoscopic ultrasound: multicentre prospective evaluation of 213 patients. Pancreatology 2005; 5: 450-456

[378] Liu CL, Fan ST, Lo CM et al. Clinico-biochemical prediction of biliary cause of acute pancreatitis in the era of endoscopic ultrasonografy. Aliment Pharmacol Ther 2005; 22: 423-431

[379] Anderson K, Brown LA, Daniel P et al. Alanine transaminase rather than abdominal ultrasound alone is an important investigation to justify cholecystectomy in patients presenting with acute pancreatitis. HPB (Oxford) 2010; 12: $342-347$

[380] Dholakia K, Pitchumoni CS, Agarwal N. How often are liver function tests normal in acute biliary pancreatitis? J Clin Gastroenterol 2004; 38: $81-83$

[381] Tenner SM, Steinberg W. The admission serum lipase:amylase ratio differentiates alcoholic from nonalcoholic acute pancreatitis. Am J Gastroenterol 1992; 87: 1755-1758

[382] Gumaste VV, Dave PB, Weissman D et al. Lipase/amylase ratio. A new index that distinguishes acute episodes of alcoholic from nonalcoholic acute pancreatitis. Gastroenterology 1991; 101: 1361-1366 
[383] King LG, Seelig CB, Ranney JE. The lipase to amylase ratio in acute pancreatitis. Am J Gastroenterol 1995; 90: 67-69

[384] Lankisch PG, Petersen M. Lipase/amylase ratio: not helpful in the early etiological differentiation of acute pancreatitis. Z Gastroenterol 1994; 32: $8-11$

[385] Pezzilli R, Billi P, Miglioli M et al. Serum amylase and lipase concentrations and lipase/amylase ratio in assessment of etiology and severity of acute pancreatitis. Dig Dis Sci 1993; 38: 1265-1269

[386] Makary MA, Duncan MD, Harmon JW et al. The role of magnetic resonance cholangiografy in the management of patients with gallstone pancreatitis. Ann Surg 2005; 241: 119-124

[387] Barlow AD, Haqq J, McCormack D et al. The role of magnetic resonance cholangiopancreatografy in the management of acute gallstone pancreatitis. Ann R Coll Surg Engl 2013; 95: 503-506

[388] Cohen ME, Slezak L, Wells CK et al. Prediction of bile duct stones and complications in gallstone pancreatitis using early laboratory trends. Am J Gastroenterol 2001; 96: 3305-3311

[389] Liu CL, Fan ST, Lo CM et al. Comparison of early endoscopic ultrasonografy and endoscopic retrograde cholangiopancreatografy in the management of acute biliary pancreatitis: a prospective randomized study. Clin Gastroenterol Hepatol 2005; 3: 1238-1244

[390] De Waele E, Op de Beeck B, De Waele B et al. Magnetic resonance cholangiopancreatografy in the preoperative assessment of patients with biliary pancreatitis. Pancreatology 2007; 7: 347-351

[391] Srinivasa S, Sammour T, McEntee B et al. Selective use of magnetic resonance cholangiopancreatografy in clinical practice may miss choledocholithiasis in gallstone pancreatitis. Can J Surg 2010; 53 : $403-407$

[392] Chan T, Yaghoubian A, Rosing D et al. Total bilirubin is a useful predictor of persisting common bile duct stone in gallstone pancreatitis. Am Surg 2008; 74: 977 - 980

[393] Signoretti M, Baccini F, Piciucchi M et al. Repeated transabdominal ultrasonografy is a simple and accurate strategy to diagnose a biliary etiology of acute pancreatitis. Pancreas 2014; 43: 1106-1110

[394] Hallal AH, Amortegui JD, Jeroukhimov IM et al. Magnetic resonance cholangiopancreatografy accurately detects common bile duct stones in resolving gallstone pancreatitis. J Am Coll Surg 2005; 200: 869-875

[395] Mofidi R, Lee AC, Madhavan KK et al. The selective use of magnetic resonance cholangiopancreatografy in the imaging of the axial biliary tree in patients with acute gallstone pancreatitis. Pancreatology 2008; 8: $55-60$

[396] Repiso A, Gomez-Rodriguez R, Garcia-Vela A et al. Endosonografic examination of the common bile duct in patients with acute biliary pancreatitis. Rev Esp Enferm Dig 2008; 100: 337 - 342

[397] Stabuc B, Drobne D, Ferkolj I et al. Acute biliary pancreatitis: detection of common bile duct stones with endoscopic ultrasound. Eur J Gastroenterol Hepatol 2008; 20: 1171 - 1175

[398] Prat F, Edery J, Meduri B et al. Early EUS of the bile duct before endoscopic sphincterotomy for acute biliary pancreatitis. Gastrointest Endosc 2001; 54: 724-729

[399] De Lisi S, Leandro G, Buscarini E. Endoscopic ultrasonografy versus endoscopic retrograde cholangiopancreatografy in acute biliary pancreatitis: a systematic review. Eur J Gastroenterol Hepatol 2011; 23: $367-374$

[400] Tanner AR, Dwarakanath AD, Tait NP. The potential impact of highquality MRI of the biliary tree on ERCP workload. Eur J Gastroenterol Hepatol 2000; 12: $773-776$

[401] Farrell RJ, Noonan N, Mahmud N et al. Potential impact of magnetic resonance cholangiopancreatografy on endoscopic retrograde cholangiopancreatografy workload and complication rate in patients referred because of abdominal pain. Endoscopy 2001; 33: 668-675
[402] Zhan X, Guo X, Chen Y et al. EUS in exploring the etiology of mild acute biliary pancreatitis with a negative finding of biliary origin by conventional radiological methods. J Gastroenterol Hepatol 2011; 26: 1500 1503

[403] Romagnuolo ], Currie G. Noninvasive vs. selective invasive biliary imaging for acute biliary pancreatitis: an economic evaluation by using decision tree analysis. Gastrointest Endosc 2005; 61: 86-97

[404] van Geenen EJ, van Santvoort HC, Besselink MG et al. Lack of consensus on the role of endoscopic retrograde cholangiografy in acute biliary pancreatitis in published metaanalyses and guidelines: a systematic review. Pancreas 2013; 42: $774-780$

[405] Tse F, Yuan Y. Early routine endoscopic retrograde cholangiopancreatografy strategy versus early conservative management strategy in acute gallstone pancreatitis. Cochrane Database Syst Rev 2012; 5: CD009779

[406] Levy MJ, Geenen JE. Idiopathic acute recurrent pancreatitis. Am J Gastroenterol 2001; 96: 2540-2555

[407] Gullo L, Migliori M, Pezzilli R et al. An update on recurrent acute pancreatitis: data from five European countries. Am J Gastroenterol 2002; 97: $1959-1962$

[408] van Brummelen SE, Venneman NG, van Erpecum KJ et al. Acute idiopathic pancreatitis: does it really exist or is it a myth? Scand J Gastroenterol Suppl 2003; 239: 117 - 122

[409] Al-Haddad M, Wallace MB. Diagnostic approach to patients with acute idiopathic and recurrent pancreatitis, what should be done? World J Gastroenterol 2008; 14: 1007 - 1010

[410] Maes B, Hastier P, Buckley MJ et al. Extensive aetiological investigations in acute pancreatitis: results of a 1-year prospective study. Eur J Gastroenterol Hepatol 1999; 11: $891-896$

[411] Frossard JL, Sosa-Valencia L, Amouyal G et al. Usefulness of endoscopic ultrasonografy in patients with "idiopathic" acute pancreatitis. Am J Med 2000; 109: 196-200

[412] Coyle W], Pineau BC, Tarnasky PR et al. Evaluation of unexplained acute and acute recurrent pancreatitis using endoscopic retrograde cholangiopancreatografy, sphincter of Oddi manometry and endoscopic ultrasound. Endoscopy 2002; 34: 617-623

[413] Wilcox CM, Varadarajulu S, Eloubeidi M. Role of endoscopic evaluation in idiopathic pancreatitis: a systematic review. Gastrointest Endosc 2006; 63: $1037-1045$

[414] Thevenot A, Bournet B, Otal P et al. Endoscopic ultrasound and magnetic resonance cholangiopancreatografy in patients with idiopathic acute pancreatitis. Dig Dis Sci 2013; 58: 2361 - 2368

[415] Kaw M, Brodmerkel G] Jr. ERCP, biliary crystal analysis, and sphincter of Oddi manometry in idiopathic recurrent pancreatitis. Gastrointest Endosc 2002; 55: $157-162$

[416] Fischer M, Hassan A, Sipe BW et al. Endoscopic retrograde cholangiopancreatografy and manometry findings in 1241 idiopathic pancreatitis patients. Pancreatology 2010; 10: 444-452

[417] Wilkinson LS, Levine TS, Smith D et al. Biliary sludge: can ultrasound reliably detect the presence of crystals in bile? Eur J Gastroenterol Hepatol 1996; 8: 999-1001

[418] lerardi E, Muscatiello N, Nacchiero M et al. Second harmonic imaging improves trans-abdominal ultrasound detection of biliary sludge in 'idiopathic' pancreatitis. Aliment Pharmacol Ther 2003; 17: $473-477$

[419] Choi CS, Ku YJ, Yoon DY et al. Harmonic ultrasonografy for the detection of microlithiasis in the gallbladder. Ultrasonografy 2014; 33: $275-282$

[420] Dahan P, Andant C, Levy P et al. Prospective evaluation of endoscopic ultrasonografy and microscopic examination of duodenal bile in the diagnosis of cholecystolithiasis in 45 patients with normal conventional ultrasonografy. Gut 1996; 38: 277-281 
[421] Ardengh JC, Malheiros CA, Rahal F et al. Microlithiasis of the gallbladder: role of endoscopic ultrasonografy in patients with idiopathic acute pancreatitis. Rev Assoc Med Bras (1992) 2010; 56: 27 - 31

[422] Norton SA, Alderson D. Endoscopic ultrasonografy in the evaluation of idiopathic acute pancreatitis. Br J Surg 2000; 87: 1650-1655

[423] Tandon M, Topazian M. Endoscopic ultrasound in idiopathic acute pancreatitis. Am J Gastroenterol 2001; 96: 705-709

[424] Queneau PE, Zeeh S, Lapeyre V et al. Feasibility of and interest in combined endoscopic ultrasonografy and biliary drainage in unexplained acute biliopancreatic disorders. Dig Dis Sci 2002; 47: 2020 - 2024

[425] Yusoff IF, Raymond G, Sahai AV. A prospective comparison of the yield of EUS in primary vs. recurrent idiopathic acute pancreatitis. Gastrointest Endosc 2004; 60: 673-678

[426] Mariani A, Arcidiacono PG, Curioni S et al. Diagnostic yield of ERCP and secretin-enhanced MRCP and EUS in patients with acute recurrent pancreatitis of unknown aetiology. Dig Liver Dis 2009; 41: 753 - 758

[427] Morris-Stiff G, Al-Allak A, Frost B et al. Does endoscopic ultrasound have anything to offer in the diagnosis of idiopathic acute pancreatitis? JOP 2009; 10: $143-146$

[428] Vila J], Vicuna M, Irisarri R et al. Diagnostic yield and reliability of endoscopic ultrasonografy in patients with idiopathic acute pancreatitis. Scand J Gastroenterol 2010; 45: 375-381

[429] Ortega AR, Gomez-Rodriguez R, Romero M et al. Prospective comparison of endoscopic ultrasonografy and magnetic resonance cholangiopancreatografy in the etiological diagnosis of "idiopathic" acute pancreatitis. Pancreas 2011; 40: 289 -294

[430] Rana SS, Bhasin DK, Rao C et al. Role of endoscopic ultrasound in idiopathic acute pancreatitis with negative ultrasound, computed tomografy, and magnetic resonance cholangiopancreatografy. Ann Gastroenterol 2012; 25: $133-137$

[431] Govil A, Agrawal MK, Agrawal D et al. Role of endoscopic ultrasonografy in patients with first episode of idiopathic acute pancreatitis. Indian J Gastroenterol 2014; 33: 241 -248

[432] Testoni PA, Mariani A, Curioni S et al. MRCP-secretin test-guided management of idiopathic recurrent pancreatitis: long-term outcomes. Gastrointest Endosc 2008; 67: 1028-1034

[433] Smith I, Ramesh J, Kyanam Kabir Baig KR et al. Emerging role of endoscopic ultrasound in the diagnostic evaluation of idiopathic pancreatitis. Am J Med Sci 2015; 350: 229-234

[434] Kim HS, Moon JH, Choi HJ et al. The role of intraductal US in the management of idiopathic recurrent pancreatitis without a definite cause on ERCP. Gastrointest Endosc 2011; 73: 1148 - 1154

[435] Denzer U, Beilenhoff U, Eickhoff A et al. S2k-Leitlinie Qualitätsanforderungen in der gastrointestinalen Endoskopie, AWMF Register Nr. 021-022. Z Gastroenterol 2015; 53: E1 - E227

[436] Elmunzer B], Waljee AK, Elta GH et al. A metaanalysis of rectal NSAIDs in the prevention of post-ERCP pancreatitis. Gut 2008; 57: 1262-1267

[437] Zheng MH, Xia HH, Chen YP. Rectal administration of NSAIDs in the prevention of post-ERCP pancreatitis: a complementary metaanalysis. Gut 2008; 57: 1632 - 1633

[438] Dai HF, Wang XW, Zhao K. Role of nonsteroidal anti-inflammatory drugs in the prevention of post-ERCP pancreatitis: a metaanalysis. Hepatobiliary Pancreat Dis Int 2009; 8: 11-16

[439] Elmunzer B], Scheiman JM, Lehman GA et al. A randomized trial of rectal indomethacin to prevent post-ERCP pancreatitis. N Engl ] Med 2012; 366: $1414-1422$

[440] Luo H, Zhao L, Leung J et al. Routine pre-procedural rectal indometacin versus selective post-procedural rectal indometacin to prevent pancreatitis in patients undergoing endoscopic retrograde cholangiopancreatografy: a multicentre, single-blinded, randomised controlled trial. Lancet 2016; 387: 2293-2301
[441] Patai A, Solymosi N, Mohacsi L et al. Indomethacin and diclofenac in the prevention of post-ERCP pancreatitis: a systematic review and metaanalysis of prospective controlled trials. Gastrointest Endosc 2017; 85: 1144 -1156.e1141

[442] Dumonceau JM, Andriulli A, Elmunzer B] et al. Prophylaxis of post-ERCP pancreatitis: European Society of Gastrointestinal Endoscopy (ESGE) Guideline - updated June 2014. Endoscopy 2014; 46: 799-815

[443] Ding X, Chen M, Huang S et al. Nonsteroidal anti-inflammatory drugs for prevention of post-ERCP pancreatitis: a metaanalysis. Gastrointest Endosc 2012; 76: $1152-1159$

[444] Elmunzer B], Higgins PD, Saini SD et al. Does rectal indomethacin eliminate the need for prophylactic pancreatic stent placement in patients undergoing high-risk ERCP? Post hoc efficacy and cost-benefit analyses using prospective clinical trial data. Am J Gastroenterol 2013; 108: $410-415$

[445] Yuhara H, Ogawa M, Kawaguchi Y et al. Pharmacologic prophylaxis of post-endoscopic retrograde cholangiopancreatografy pancreatitis: protease inhibitors and NSAIDs in a metaanalysis. J Gastroenterol 2014; 49: 388-399

[446] Yaghoobi M, Rolland S, Waschke KA et al. Metaanalysis: rectal indomethacin for the prevention of post-ERCP pancreatitis. Aliment Pharmacol Ther 2013; 38: 995 - 1001

[447] Sun HL, Han B, Zhai HP et al. Rectal NSAIDs for the prevention of postERCP pancreatitis: a metaanalysis of randomized controlled trials. Surgeon 2014; 12: 141 - 147

[448] Sethi S, Sethi N, Wadhwa V et al. A metaanalysis on the role of rectal diclofenac and indomethacin in the prevention of post-endoscopic retrograde cholangiopancreatografy pancreatitis. Pancreas 2014; 43: $190-197$

[449] Choudhary A, Bechtold ML, Arif M et al. Pancreatic stents for prophylaxis against post-ERCP pancreatitis: a metaanalysis and systematic review. Gastrointest Endosc 2011; 73: 275-282

[450] Mazaki T, Masuda H, Takayama T. Prophylactic pancreatic stent placement and post-ERCP pancreatitis: a systematic review and metaanalysis. Endoscopy 2010; 42: $842-853$

[451] Singh P, Das A, Isenberg G et al. Does prophylactic pancreatic stent placement reduce the risk of post-ERCP acute pancreatitis? A metaanalysis of controlled trials. Gastrointest Endosc 2004; 60: 544-550

[452] Akbar A, Abu Dayyeh BK, Baron TH et al. Rectal nonsteroidal anti-inflammatory drugs are superior to pancreatic duct stents in preventing pancreatitis after endoscopic retrograde cholangiopancreatografy: a network metaanalysis. Clin Gastroenterol Hepatol 2013; 11: 778-783

[453] Dumonceau JM, Andriulli A, Deviere J et al. European society of gastrointestinal endoscopy (ESGE) guideline: prophylaxis of post-ERCP pancreatitis. Endoscopy 2010; 42: 503 - 515

[454] Lee TH, Moon JH, Choi HJ et al. Prophylactic temporary 3F pancreatic duct stent to prevent post-ERCP pancreatitis in patients with a difficult biliary cannulation: a multicenter, prospective, randomized study. Gastrointest Endosc 2012; 76: 578 - 585

[455] Cha SW, Leung WD, Lehman GA et al. Does leaving a main pancreatic duct stent in place reduce the incidence of precut biliary sphincterotomy-associated pancreatitis? A randomized, prospective study. Gastrointest Endosc 2013; 77: 209-216

[456] Afghani E, Akshintala VS, Khashab MA et al. 5-Fr vs. 3-Fr pancreatic stents for the prevention of post-ERCP pancreatitis in high-risk patients: a systematic review and network metaanalysis. Endoscopy 2014; 46: 573 - 580

[457] Rocca R, De Angelis C, Castellino F et al. EUS diagnosis and simultaneous endoscopic retrograde cholangiografy treatment of common bile duct stones by using an oblique-viewing echoendoscope. Gastrointest Endosc 2006; 63: 479-484 
[458] Ross WA, Wasan SM, Evans DB et al. Combined EUS with FNA and ERCP for the evaluation of patients with obstructive jaundice from presumed pancreatic malignancy. Gastrointest Endosc 2008; 68: 461 - 466

[459] Ascunce G, Ribeiro A, Rocha-Lima C et al. Single-session endoscopic ultrasonografy and endoscopic retrograde cholangiopancreatografy for evaluation of pancreaticobiliary disorders. Surg Endosc 2010; 24 : $1447-1450$

[460] Aslanian HR, Estrada JD, Rossi F et al. Endoscopic ultrasound and endoscopic retrograde cholangiopancreatografy for obstructing pancreas head masses: combined or separate procedures? J Clin Gastroenterol 2011; 45: $711-713$

[461] Vila J], Kutz M, Goni S et al. Endoscopic and anesthetic feasibility of EUS and ERCP combined in a single session versus two different sessions. World J Gastrointest Endosc 2011; 3: 57-61

[462] Camus M, Trouilloud I, Villacis AL et al. Effectiveness of combined endoscopic ultrasound-guided fine-needle aspiration biopsy and stenting in patients with suspected pancreatic cancer. Eur J Gastroenterol Hepatol 2012; 24: 1281 - 1287

[463] Benjaminov F, Stein A, Lichtman G et al. Consecutive versus separate sessions of endoscopic ultrasound (EUS) and endoscopic retrograde cholangiopancreatografy (ERCP) for symptomatic choledocholithiasis. Surg Endosc 2013; 27: 2117 - 2121

[464] Gornals JB, Moreno R, Castellote J et al. Single-session endosonografy and endoscopic retrograde cholangiopancreatografy for biliopancreatic diseases is feasible, effective and cost beneficial. Dig Liver Dis 2013; 45: $578-583$

[465] Kawakubo K, Kawakami H, Kuwatani M et al. Safety and utility of single-session endoscopic ultrasonografy and endoscopic retrograde cholangiopancreatografy for the evaluation of pancreatobiliary diseases. Gut Liver 2014; 8: 329-332

[466] van Geenen EJ, van der Peet DL, Mulder C] et al. Recurrent acute biliary pancreatitis: the protective role of cholecystectomy and endoscopic sphincterotomy. Surg Endosc 2009; 23: 950-956

[467] van Baal MC, Besselink MG, Bakker OJ et al. Timing of cholecystectomy after mild biliary pancreatitis: a systematic review. Ann Surg 2012; 255: $860-866$

[468] Yadav D, O'Connell M, Papachristou GI. Natural history following the first attack of acute pancreatitis. Am J Gastroenterol 2012; 107: 1096 1103

[469] Gurusamy KS, Nagendran M, Davidson BR. Early versus delayed laparoscopic cholecystectomy for acute gallstone pancreatitis. Cochrane Database Syst Rev 2013; 9: CD010326

[470] da Costa DW, Bouwense SA, Schepers NJ et al. Same-admission versus interval cholecystectomy for mild gallstone pancreatitis (PONCHO): a multicentre randomised controlled trial. Lancet 2015; 386: 1261 1268

[471] Brauer BC, Chen YK, Fukami N et al. Single-operator EUS-guided cholangiopancreatografy for difficult pancreaticobiliary access (with video). Gastrointest Endosc 2009; 70: 471 - 479

[472] Shah JN, Marson F, Weilert F et al. Single-operator, single-session EUS-guided anterograde cholangiopancreatografy in failed ERCP or inaccessible papilla. Gastrointest Endosc 2012; 75: 56-64

[473] Park DH, Kim MH, Lee SS et al. Accuracy of magnetic resonance cholangiopancreatografy for locating hepatolithiasis and detecting accompanying biliary strictures. Endoscopy 2004; 36: 987 -992

[474] Mori T, Sugiyama M, Atomi Y. Gallstone disease: management of intrahepatic stones. Best Pract Res Clin Gastroenterol 2006; 20: 1117 1137

[475] Erden A, Haliloglu N, Genc Y et al. Diagnostic value of T1-weighted gradient-echo in-phase images added to MRCP in differentiation of hepatolithiasis and intrahepatic pneumobilia. Am J Roentgenol 2014; 202: $74-82$
[476] Suzuki Y, Mori T, Yokoyama M et al. Hepatolithiasis: analysis of Japanese nationwide surveys over a period of 40 years. J Hepatobiliary Pancreat Sci 2014; 21: 617-622

[477] Kusano T, Isa T, Ohtsubo M et al. Natural progression of untreated hepatolithiasis that shows no clinical signs at its initial presentation. J Clin Gastroenterol 2001; 33: 114-117

[478] Neuhaus H. Intrahepatic stones: the percutaneous approach. Can J Gastroenterol 1999; 13: 467-472

[479] Di Carlo I, Sauvanet A, Belghiti J. Intrahepatic lithiasis: a Western experience. Surg Today 2000; 30: 319-322

[480] Maetani I, Ishiguro J, Ogawa S et al. Percutaneous choledochoscopic treatment of intrahepatic stones, including management of associated biliary stenoses. Endoscopy 1999; 31: $456-459$

[481] Adamek HE, Schneider AR, Adamek MU et al. Treatment of difficult intrahepatic stones by using extracorporeal and intracorporeal lithotripsy techniques: 10 years' experience in 55 patients. Scand J Gastroenterol 1999; 34: 1157-1161

[482] Jeng KS, Sheen IS, Yang FS. Are modified procedures significantly better than conventional procedures in percutaneous transhepatic treatment for complicated right hepatolithiasis with intrahepatic biliary strictures? Scand J Gastroenterol 2002; 37: 597 - 601

[483] Wu YW, Jian YP, Liang JS et al. The treatment of intrahepatic calculosis by applying helix hydro-jet lithotripsy under video choledochoscope: a report of 30 cases. Langenbecks Arch Surg 2006; 391: 355 - 358

[484] May GR, Sutherland LR, Shaffer EA. Efficacy of bile acid therapy for gallstone dissolution: a metaanalysis of randomized trials. Aliment Pharmacol Ther 1993; 7: 139-148

[485] Paumgartner G. Strategies in the treatment of gallstone disease. Working team report. Gastroenterology International 1993; 6: 65-75

[486] Pauletzki ], Sailer C, Klüppelberg U et al. Gallbladder emptying determines early gallstone clearance after shock-wave lithotripsy. Gastroenterology 1994; 107: 1496-1502

[487] Jazrawi RP, Pigozzi MG, Galatola G et al. Optimum bile acid treatment for rapid gall stone dissolution. Gut 1992; 33: 381 - 386

[488] Janssen J, Johanns W, Weickert $U$ et al. Long-term results after successful extracorporeal gallstone lithotripsy: outcome of the first 120 stone-free patients. Scand J Gastroenterol 2001; 36: 314-317

[489] Tsumita R, Sugiura N, Abe A et al. Long-term evaluation of extracorporeal shock-wave lithotripsy for cholesterol gallstones. J Gastroenterol Hepatol 2001; 16: $93-99$

[490] Venneman NG, van Berge-Henegouwen GP, Portincasa P et al. Absence of apolipoprotein E4 genotype, good gallbladder motility and presence of solitary stones delay rather than prevent gallstone recurrence after extracorporeal shock wave lithotripsy. J Hepatol 2001; 35: 10 - 16

[491] Rabenstein T, Radespiel-Troger M, Hopfner L et al. Ten years experience with piezoelectric extracorporeal shockwave lithotripsy of gallbladder stones. Eur J Gastroenterol Hepatol 2005; 17: 629-639

[492] Carrilho-Ribeiro L, Pinto-Correia A, Velosa J et al. A ten-year prospective study on gallbladder stone recurrence after successful extracorporeal shock-wave lithotripsy. Scand J Gastroenterol 2006; 41: 338 - 342

[493] Petroni ML, Jazrawi RP, Goggin PM et al. Characteristics of recurrent gallstones following non-surgical treatment: implications for retreatment. Eur J Gastroenterol Hepatol 1991; 3: 473 -478

[494] Sackmann M, Pauletzki ], Aydemir U et al. Efficacy and safety of ursodeoxycholic acid for dissolution of gallstone fragments: comparison with the combination of ursodeoxycholic acid and chenodeoxycholic acid. Hepatology 1991; 14: 1136-1141

[495] Sackmann M, Niller H, Klüppelberg U et al. Gallstone recurrence after shock-wave therapy. Gastroenterology 1994; 106: 225-230

[496] Lanzini A, Jazrawi RP, Kupfer RM et al. Gallstone recurrence after medical dissolution. An overestimated threat? J Hepatol 1986; 3: 241 246 
[497] Pelletier G, Raymond JM, Capdeville R et al. Gallstone recurrence after successful lithotripsy. J Hepatol 1995; 23: 420-423

[498] Pauletzki ], Althaus R, Holl J et al. Gallbladder emptying and gallstone formation: a prospective study on gallstone recurrence. Gastroenterology 1996; 111: $765-771$

[499] Ochi H, Tazuma S, Kajihara T et al. Factors affecting gallstone recurrence after successful extracorporeal shock wave lithotripsy. J Clin Gastroenterol 2000; 31: 230-232

[500] Petroni ML, Jazrawi RP, Pazzi P et al. Risk factors for the development of gallstone recurrence following medical dissolution. The British-Italian Gallstone Study Group. Eur J Gastroenterol Hepatol 2000; 12: 695 700

[501] Tazuma S, Nishioka T, Ochi H et al. Impaired gallbladder mucosal function in aged gallstone patients suppresses gallstone recurrence after successful extracorporeal shockwave lithotripsy. J Gastroenterol Hepatol 2003; 18: 157-161

[502] Adamek HE, Rochlitz C, von Bubnoff AC et al. Predictions and associations of cholecystectomy in patients with cholecystolithiasis treated with extracorporeal shock wave lithotripsy. Dig Dis Sci 2004; 49: $1938-1942$

[503] Schmieder G, Stankov G, Zerle G et al. Observer-blind study with metamizole versus tramadol and butylscopolamine in acute biliary colic pain. Arzneimittelforschung 1993; 43: 1216-1221

[504] Cuer JC, Dapoigny M, Ajmi S et al. Effects of buprenorphine on motor activity of the sphincter of Oddi in man. Eur J Clin Pharmacol 1989; 36: 203-204

[505] Staritz M, Poralla T, Manns M et al. Effect of modern analgesic drugs (tramadol, pentazocine, and buprenorphine) on the bile duct sphincter in man. Gut 1986; 27: 567-569

[506] Wu SD, Zhang ZH, jin JZ et al. Effects of narcotic analgesic drugs on human Oddi's sphincter motility. World J Gastroenterol 2004; 10: 2901-2904

[507] Hassel B. Treatment of biliary colic with nitroglycerin. Lancet 1993; 342: 1305

[508] Goldman G, Kahn P], Alon R et al. Biliary colic treatment and acute cholecystitis prevention by prostaglandin inhibitor. Dig Dis Sci 1989; 34: 809-811

[509] Akriviadis EA, Hatzigavriel M, Kapnias D et al. Treatment of biliary colic with diclofenac: a randomized, double-blind, placebo-controlled study. Gastroenterology 1997; 113: 225-231

[510] Thornell E, Jansson R, Svanvik J. Indomethacin intravenously - a new way for effective relief of biliary pain: a double-blind study in man. Surgery 1981; 90: $468-472$

[511] Thornell E, Nilsson B, Jansson R et al. Effect of short-term indomethacin treatment on the clinical course of acute obstructive cholecystitis. Eur J Surg 1991; 157: $127-130$

[512] Kumar A, Deed JS, Bhasin B et al. Comparison of the effect of diclofenac with hyoscine-N-butylbromide in the symptomatic treatment of acute biliary colic. ANZ J Surg 2004; 74: 573-576

[513] Mazeh H, Mizrahi I, Dior U et al. Role of antibiotic therapy in mild acute calculus cholecystitis: a prospective randomized controlled trial. World J Surg 2012; 36: 1750 - 1759

[514] Yokoe M, Hata J, Takada T et al. Tokyo guidelines 2018 diagnostic criteria and severity grading of acute cholecystitis (with videos). J Hepatobiliary Pancreat Sci 2018; 25: 41- 54

[515] Okamoto K, Suzuki K, Takada T et al. Tokyo guidelines 2018: flowchart for the management of acute cholecystitis. J Hepatobiliary Pancreat Sci 2018; 25: 55-72

[516] Park TY, Choi JS, Song TJ et al. Early oral antibiotic switch compared with conventional intravenous antibiotic therapy for acute cholangitis with bacteremia. Dig Dis Sci 2014; 59: 2790-2796
[517] Regimbeau JM, Fuks D, Pautrat K et al. Effect of postoperative antibiotic administration on postoperative infection following cholecystectomy for acute calculous cholecystitis: a randomized clinical trial. JAMA 2014; 312: 145-154

[518] van Lent AU, Bartelsman JF, Tytgat GN et al. Duration of antibiotic therapy for cholangitis after successful endoscopic drainage of the biliary tract. Gastrointest Endosc 2002; 55: 518 - 522

[519] Bodmann KF, Grabein B. und die Expertenkommission der Paul-Ehrlich-Gesellschaft für Chemotherapie e. V. Empfehlungen zur kalkulierten parenteralen Initialtherapie bakterieller Erkrankungen bei Erwachsenen - Update 2010. Chemother J 2010; 19: 179-255

[520] Venneman NG, Besselink MG, Keulemans YC et al. Ursodeoxycholic acid exerts no beneficial effect in patients with symptomatic gallstones awaiting cholecystectomy. Hepatology 2006; 43: 1276-1283

[521] Ransohoff DF, Gracie WA. Management of patients with symptomatic gallstones: a quantitative analysis. Am J Med 1990; 88: 154- 160

[522] Bates T, Ebbs SR, Harrison M et al. Influence of cholecystectomy on symptoms. Br J Surg 1991; 78: 964-967

[523] Plaisier PW, van der Hul RL, Nijs HG et al. The course of biliary and gastrointestinal symptoms after treatment of uncomplicated symptomatic gallstones: results of a randomized study comparing extracorporeal shock wave lithotripsy with conventional cholecystectomy. Am J Gastroenterol 1994; 89: 739-744

[524] Qureshi MA, Burke PE, Brindley NM et al. Post-cholecystectomy symptoms after laparoscopic cholecystectomy. Ann R Coll Surg Engl 1993; 75: $349-353$

[525] Lublin M, Crawford DL, Hiatt JR et al. Symptoms before and after laparoscopic cholecystectomy for gallstones. Am Surg 2004; 70: 863-866

[526] Vetrhus M, Berhane T, Soreide O et al. Pain persists in many patients five years after removal of the gallbladder: observations from two randomized controlled trials of symptomatic, noncomplicated gallstone disease and acute cholecystitis. J Gastrointest Surg 2005; 9: $826-831$

[527] Lamberts MP, Den Oudsten BL, Keus F et al. Patient-reported outcomes of symptomatic cholelithiasis patients following cholecystectomy after at least 5 years of follow-up: a long-term prospective cohort study. Surg Endosc 2014; 28: 3443-3450

[528] Lamberts MP, Den Oudsten BL, Gerritsen J] et al. Prospective multicentre cohort study of patient-reported outcomes after cholecystectomy for uncomplicated symptomatic cholecystolithiasis. Br J Surg 2015; 102: 1402-1409

[529] Mangram AJ, Horan TC, Pearson ML et al. Guideline for prevention of surgical site infection, 1999. Centers for disease control and prevention $(C D C)$ hospital infection control practices advisory committee. Am J Infect Control 1999; 27: 97-132

[530] National Nosocomial Infections Surveillance S. National nosocomial infections surveillance (NNIS) system report, data summary from January 1992 through June 2004, issued October 2004. Am J Infect Control 2004; 32: 470-485

[531] Goldfaden A, Birkmeyer JD. Evidence-based practice in laparoscopic surgery: perioperative care. Surg Innov 2005; 12: 51-61

[532] Darkahi B, Videhult P, Sandblom G et al. Effectiveness of antibiotic prophylaxis in cholecystectomy: a prospective population-based study of 1171 cholecystectomies. Scand J Gastroenterol 2012; 47: 1242 1246

[533] Enochsson L, Thulin A, Osterberg J et al. The Swedish registry of gallstone surgery and endoscopic retrograde cholangiopancreatografy (GallRiks): a nationwide registry for quality assurance of gallstone surgery. JAMA Surg 2013; 148: $471-478$

[534] de Mestral C, Nathens AB. Prevention, diagnosis, and management of surgical site infections: relevant considerations for critical care medicine. Crit Care Clin 2013; 29: 887-894 
[535] Graham HE, Vasireddy A, Nehra D. A national audit of antibiotic prophylaxis in elective laparoscopic cholecystectomy. Ann R Coll Surg Engl 2014; 96: $377-380$

[536] Sanabria A, Dominguez LC, Valdivieso E et al. Antibiotic prophylaxis for patients undergoing elective laparoscopic cholecystectomy. Cochrane Database Syst Rev 2010; 12: CD005265

[537] Yan RC, Shen SQ, Chen ZB et al. The role of prophylactic antibiotics in laparoscopic cholecystectomy in preventing postoperative infection: a metaanalysis. J Laparoendosc Adv Surg Tech A 2011; 21: 301 - 306

[538] Pasquali S, Boal M, Griffiths EA et al. Metaanalysis of perioperative antibiotics in patients undergoing laparoscopic cholecystectomy. $\mathrm{Br}$ J Surg 2016; 103: 27 - 34; discussion 34

[539] Jaafar G, Persson G, Svennblad B et al. Outcomes of antibiotic prophylaxis in acute cholecystectomy in a population-based gallstone surgery registry. Br J Surg 2014; 101: 69-73

[540] Naqvi MA, Mehraj A, Ejaz R et al. Role of prophylactic antibiotics in low risk elective laparoscopic cholecystectomy: is there a need? J Ayub Med Coll Abbottabad 2013; 25: 172 - 174

[541] Matsui Y, Satoi S, Kaibori M et al. Antibiotic prophylaxis in laparoscopic cholecystectomy: a randomized controlled trial. PLoS One 2014; 9 : e106702

[542] Wacha H, Hoyme U, Isenmann R et al. Perioperative Antibiotikaprophylaxe. Chemother J 2010; 19: 70-84

[543] Knebel P, Weigand MA, Buchler MW et al. Evidenzbasierte Antibiotikaprophylaxe in der Allgemein- und Viszeralchirurgie. Chirurg 2011; 82: $227-234$

[544] Classen DC, Evans RS, Pestotnik SL et al. The timing of prophylactic administration of antibiotics and the risk of surgical-wound infection. N Engl J Med 1992; 326: 281 - 286

[545] Bates T, Siller G, Crathern BC et al. Timing of prophylactic antibiotics in abdominal surgery: trial of a pre-operative versus an intra-operative first dose. $\mathrm{Br}$ J Surg 1989; 76: $52-56$

[546] Weber WP, Marti WR, Zwahlen M et al. The timing of surgical antimicrobial prophylaxis. Ann Surg 2008; 247: 918-926

[547] Bowater RJ, Stirling SA, Lilford R]. Is antibiotic prophylaxis in surgery a generally effective intervention? Testing a generic hypothesis over a set of metaanalyses. Ann Surg 2009; 249: 551 - 556

[548] Ransohoff DF, Gracie WA, Wolfenson LB et al. Prophylactic cholecystectomy or expectant management for silent gallstones. A decision analysis to assess survival. Ann Intern Med 1983; 99: 199-204

[549] Randi G, Franceschi S, La Vecchia C. Gallbladder cancer worldwide: geografical distribution and risk factors. Int J Cancer 2006; 118: 1591 1602

[550] Maringhini A, Moreau JA, Melton L] et al. Gallstones, gallbladder cancer, and other gastrointestinal malignancies. An epidemiologic study in Rochester, Minnesota. Ann Intern Med 1987; 107: 30 - 35

[551] Sheth S, Bedford A, Chopra S. Primary gallbladder cancer: recognition of risk factors and the role of prophylactic cholecystectomy. Am J Gastroenterol 2000; 95: 1402-1410

[552] Pagliarulo M, Fornari F, Fraquelli M et al. Gallstone disease and related risk factors in a large cohort of diabetic patients. Dig Liver Dis 2004; 36: $130-134$

[553] Mendez-Sanchez N, Bahenaaponte ], Chavez-Tapia NC et al. Strong association between gallstones and cardiovascular disease. Am J Gastroenterol 2005; 100: $827-830$

[554] Lynen-Jansen P. Klug entscheiden: ... in der Gastroenterologie. Dtsch Arztebl 2016; 113: A-1385/B-1168/C-1148

[555] Stephen AE, Berger DL. Carcinoma in the porcelain gallbladder: a relationship revisited. Surgery 2001; 129: 699-703

[556] Cunningham SC, Alexander HR. Porcelain gallbladder and cancer: ethnicity explains a discrepant literature? Am J Med 2007; 120: e17 -e18
[557] Cariati A, Piromalli E, Cetta F. Gallbladder cancers: associated conditions, histological types, prognosis, and prevention. Eur J Gastroenterol Hepatol 2014; 26: 562 - 569

[558] Hundal R, Shaffer EA. Gallbladder cancer: epidemiology and outcome. Clin Epidemiol 2014; 6: 99 - 109

[559] Law CHL, McKay D, Tandan VR. Gallstone disease. In: McDonald JWD, Burroughs A, Feagan BG, Hrsg Evidence-based Gastroenterology and Child Health. Oxford: Blackwell Publishing; 2004: 311 - 320

[560] Lai CH, Lau WY. Gallbladder cancer-a comprehensive review. Surgeon 2008; 6: $101-110$

[561] Jørgensen T, Jensen KH. Polyps in the gallbladder. A prevalence study. Scand J Gastroenterol 1990; 25: 281 - 286

[562] Chen CY, Lu CL, Chang FY et al. Risk factors for gallbladder polyps in the Chinese population. Am J Gastroenterol 1997; 92: 2066-2068

[563] Okamoto M, Okamoto H, Kitahara F et al. Ultrasonografic evidence of association of polyps and stones with gallbladder cancer. Am J Gastroenterol 1999; 94: 446-450

[564] Myers RP, Shaffer EA, Beck PL. Gallbladder polyps: epidemiology, natural history and management. Can J Gastroenterol 2002; 16: 187 194

[565] Heyder N, Gunter E, Giedl ] et al. Polypoide Läsionen der Gallenblase. Dtsch Med Wochenschr 1990; 115: $243-247$

[566] Kratzer W, Schmid A, Akinli AS et al. Gallenblasenpolypen: Prävalenz und Risikofaktoren. Ultraschall in Med 2011; 32 (Suppl. 1): S68-S73

[567] Yang HL, Sun YG, Wang Z. Polypoid lesions of the gallbladder: diagnosis and indications for surgery. Br J Surg 1992; 79: 227-229

[568] Mainprize KS, Gould SW, Gilbert JM. Surgical management of polypoid lesions of the gallbladder. Br J Surg 2000; 87: 414-417

[569] Terzi C, Sokmen S, Seckin S et al. Polypoid lesions of the gallbladder: report of 100 cases with special reference to operative indications. Surgery 2000; 127: 622-627

[570] Lee KF, Wong J, Li JC et al. Polypoid lesions of the gallbladder. Am J Surg 2004; 188: 186-190

[571] Park HY, Oh SH, Lee KH et al. Is cholecystectomy a reasonable treatment option for simple gallbladder polyps larger than $10 \mathrm{~mm}$ ? World Gastroenterol 2015; 21: 4248-4254

[572] Moriguchi H, Tazawa J, Hayashi Y et al. Natural history of polypoid lesions in the gall bladder. Gut 1996; 39: $860-862$

[573] Csendes A, Burgos AM, Csendes P et al. Late follow-up of polypoid lesions of the gallbladder smaller than $10 \mathrm{~mm}$. Ann Surg 2001; 234: $657-660$

[574] Elmasry M, Lindop D, Dunne DF et al. The risk of malignancy in ultrasound detected gallbladder polyps: A systematic review. Int J Surg 2016; 33 Pt A: 28-35

[575] Wiles R, Varadpande M, Muly S et al. Growth rate and malignant potential of small gallbladder polyps-systematic review of evidence. Surgeon 2014; 12: $221-226$

[576] Bhatt NR, Gillis A, Smoothey CO et al. Evidence based management of polyps of the gall bladder: A systematic review of the risk factors of malignancy. Surgeon 2016; 14: 278-286

[577] Koga A, Watanabe K, Fukuyama T et al. Diagnosis and operative indications for polypoid lesions of the gallbladder. Arch Surg 1988; 123 : $26-29$

[578] Aldouri AQ, Malik HZ, Waytt J et al. The risk of gallbladder cancer from polyps in a large multiethnic series. Eur J Surg Oncol 2009; 35: 48- 51

[579] Matos AS, Baptista HN, Pinheiro C et al. Gallbladder polyps: how should they be treated and when? Rev Assoc Med Bras (1992) 2010; 56 : 318 321

[580] Stinton LM, Shaffer EA. Epidemiology of gallbladder disease: cholelithiasis and cancer. Gut Liver 2012; 6: 172 -187 
[581] Wiles R, Thoeni RF, Barbu ST et al. Management and follow-up of gallbladder polyps: Joint guidelines between the European Society of Gastrointestinal and Abdominal Radiology (ESGAR), European Association for Endoscopic Surgery and other Interventional Techniques (EAES), International Society of Digestive Surgery - European Federation (EFISDS) and European Society of Gastrointestinal Endoscopy (ESGE). Eur Radiol 2017; 27: 3856-3866

[582] Kubota K, Bandai $Y$, Noie T et al. How should polypoid lesions of the gallbladder be treated in the era of laparoscopic cholecystectomy? Surgery 1995; 117: 481-487

[583] Persley KM. Gallbladder polyps. Curr Treat Options Gastroenterol 2005; 8: 105-108

[584] Morera-Ocon F], Ballestin-Vicente J, Calatayud-Blas AM et al. Surgical indications in gallbladder polyps. Cir Esp 2013; 91: 324-330

[585] Gumbs AA, Hoffman JP. Laparoscopic completion radical cholecystectomy for T2 gallbladder cancer. Surg Endosc 2010; 24: 3221 - 3223

[586] Gumbs AA, Jarufe N, Gayet B. Minimally invasive approaches to extrapancreatic cholangiocarcinoma. Surg Endosc 2013; 27: 406-414

[587] Sugiyama M, Atomi Y, Yamato T. Endoscopic ultrasonografy for differential diagnosis of polypoid gall bladder lesions: analysis in surgical and follow up series. Gut 2000; 46: 250-254

[588] Azuma T, Yoshikawa T, Araida T et al. Differential diagnosis of polypoid lesions of the gallbladder by endoscopic ultrasonografy. Am J Surg 2001; 181: $65-70$

[589] Cheon YK, Cho WY, Lee TH et al. Endoscopic ultrasonografy does not differentiate neoplastic from non-neoplastic small gallbladder polyps. World J Gastroenterol 2009; 15: 2361-2366

[590] Kim SY, Cho JH, Kim EJ et al. The efficacy of real-time colour doppler flow imaging on endoscopic ultrasonografy for differential diagnosis between neoplastic and non-neoplastic gallbladder polyps. Eur Radiol 2017. doi:10.1007/s00330-017-5175-3

[591] Park CH, Chung M], Oh TG et al. Differential diagnosis between gallbladder adenomas and cholesterol polyps on contrast-enhanced harmonic endoscopic ultrasonografy. Surg Endosc 2013; 27: 1414-1421

[592] Gillen S, Michalski CW, Schuster T et al. Simultaneous/incidental cholecystectomy during gastric/esophageal resection: systematic analysis of risks and benefits. World J Surg 2010; 34: 1008-1014

[593] Rehnberg O, Haglund U. Gallstone disease following antrectomy and gastroduodenostomy with or without vagotomy. Ann Surg 1985; 201: $315-318$

[594] Pezzolla F, Lantone G, Guerra V et al. Influence of the method of digestive tract reconstruction on gallstone development after total gastrectomy for gastric cancer. Am J Surg 1993; 166: 6-10

[595] Farsi M, Bernini M, Bencini L et al. The CHOLEGAS study: multicentric randomized, blinded, controlled trial of gastrectomy plus prophylactic cholecystectomy versus gastrectomy only, in adults submitted to gastric cancer surgery with curative intent. Trials 2009; 10: 32

[596] Bernini M, Bencini L, Sacchetti R et al. The Cholegas Study: safety of prophylactic cholecystectomy during gastrectomy for cancer: preliminary results of a multicentric randomized clinical trial. Gastric Cancer 2013; 16: 370-376

[597] Worni M, Guller U, Shah A et al. Cholecystectomy concomitant with laparoscopic gastric bypass: a trend analysis of the nationwide inpatient sample from 2001 to 2008. Obes Surg 2012; 22: 220-229

[598] Tsirline VB, Keilani ZM, El Djouzi S et al. How frequently and when do patients undergo cholecystectomy after bariatric surgery? Surg Obes Relat Dis 2014; 10: 313-321

[599] Warschkow R, Tarantino I, Ukegjini K et al. Concomitant cholecystectomy during laparoscopic Roux-en-Y gastric bypass in obese patients is not justified: a metaanalysis. Obes Surg 2013; 23: 397-407

[600] Thompson JS. The role of prophylactic cholecystectomy in the shortbowel syndrome. Arch Surg 1996; 131: 556-559; discussion 559-560
[601] Chew SS, Ngo TQ, Douglas PR et al. Cholecystectomy in patients with Crohn's ileitis. Dis Colon Rectum 2003; 46: 1484-1488

[602] Parente F, Pastore L, Bargiggia S et al. Incidence and risk factors for gallstones in patients with inflammatory bowel disease: a large casecontrol study. Hepatology 2007; 45: 1267-1274

[603] Navaneethan U, Parasa S, Venkatesh PG et al. Impact of inflammatory bowel disease on post-cholecystectomy complications and hospitalization costs: a Nationwide Inpatient Sample study. J Crohns Colitis 2013; 7: e164-e170

[604] Lo CM, Liu CL, Fan ST et al. Prospective randomized study of early versus delayed laparoscopic cholecystectomy for acute cholecystitis. Ann Surg 1998; 227: $461-467$

[605] Lai PB, Kwong KH, Leung KL et al. Randomized trial of early versus delayed laparoscopic cholecystectomy for acute cholecystitis. Br J Surg 1998; 85: $764-767$

[606] Madan AK, Aliabadi-Wahle S, Tesi D et al. How early is early laparoscopic treatment of acute cholecystitis? Am J Surg 2002; 183: 232-236

[607] Johansson M, Thune A, Blomqvist A et al. Management of acute cholecystitis in the laparoscopic era: results of a prospective, randomized clinical trial. J Gastrointest Surg 2003; 7: 642-645

[608] Papi C, Catarci M, D’Ambrosio L et al. Timing of cholecystectomy for acute calculous cholecystitis: a metaanalysis. Am J Gastroenterol 2004; 99: $147-155$

[609] Kolla SB, Aggarwal S, Kumar A et al. Early versus delayed laparoscopic cholecystectomy for acute cholecystitis: a prospective randomized trial. Surg Endosc 2004; 18: 1323 - 1327

[610] Gurusamy KS, Samraj K. Early versus delayed laparoscopic cholecystectomy for acute cholecystitis. Cochrane Database Syst Rev 2006; 4: CD005440

[611] Gurusamy K, Samraj K, Gluud C et al. Metaanalysis of randomized controlled trials on the safety and effectiveness of early versus delayed laparoscopic cholecystectomy for acute cholecystitis. Br J Surg 2010; 97: $141-150$

[612] Stevens KA, Chi A, Lucas LC et al. Immediate laparoscopic cholecystectomy for acute cholecystitis: no need to wait. Am J Surg 2006; 192: $756-761$

[613] Gutt CN. Akute Cholezystitis: primär konservatives oder operatives Vorgehen? Chirurg 2013; 84: 185-190

[614] Banz V, Gsponer T, Candinas D et al. Population-based analysis of 4113 patients with acute cholecystitis: defining the optimal time-point for laparoscopic cholecystectomy. Ann Surg 2011; 254: 964-970

[615] Roulin D, Saadi A, Di Mare L et al. Early versus delayed cholecystectomy for acute cholecystitis, are the 72 hours still the rule?: A randomized trial. Ann Surg 2016; 264: 717-722

[616] Wilson RG, Macintyre IM, Nixon S] et al. Laparoscopic cholecystectomy as a safe and effective treatment for severe acute cholecystitis. Br Med | 1992; 305: 394-396

[617] Clavien PA, Richon J, Burgan S et al. Gallstone ileus. Br ] Surg 1990; 77: $737-742$

[618] Bedirli A, Sakrak O, Sozuer EM et al. Factors effecting the complications in the natural history of acute cholecystitis. Hepatogastroenterology 2001; 48: $1275-1278$

[619] Wakai T, Shirai Y, Yokoyama $\mathrm{N}$ et al. Early gallbladder carcinoma does not warrant radical resection. Br J Surg 2001; 88: 675-678

[620] Misra S, Chaturvedi A, Misra NC et al. Carcinoma of the gallbladder. Lancet Oncol 2003; 4: 167-176

[621] Ott R, Hauss ]. Notwendigkeit und Ausdehnung der Lymphadenektomie beim Gallenblasenkarzinom. Zentralbl Chir 2006; 131: 474-477

[622] Götze TO, Paolucci V. Benefits of reoperation of T2 and more advanced incidental gallbladder carcinoma: analysis of the German registry. Ann Surg 2008; 247: 104-108 
[623] Götze TO, Paolucci V. Immediate re-resection of T1 incidental gallbladder carcinomas: a survival analysis of the German Registry. Surg Endosc 2008; 22: 2462-2465

[624] You DD, Lee HG, Paik KY et al. What is an adequate extent of resection for T1 gallbladder cancers? Ann Surg 2008; 247: 835-838

[625] Cavallaro A, Piccolo G, Panebianco V et al. Incidental gallbladder cancer during laparoscopic cholecystectomy: managing an unexpected finding. World J Gastroenterol 2012; 18: 4019-4027

[626] Götze TO, Paolucci V. Inzidentelle T1b- bis T3-Gallenblasenkarzinome. Chirurg 2014; 85: $131-138$

[627] Benson AB 3rd, Abrams TA, Ben-Josef E et al. NCCN clinical practice guidelines in oncology: hepatobiliary cancers. J Natl Compr Canc Netw 2009; 7: $350-391$

[628] Valle JW, Borbath I, Khan SA et al. Biliary cancer: ESMO clinical practice guidelines for diagnosis, treatment and follow-up. Ann Oncol 2016; 27: v28-v37

[629] Fuks D, Regimbeau JM, Le Treut YP et al. Incidental gallbladder cancer by the AFC-GBC-2009 Study Group. World J Surg 2011; 35: 1887 1897

[630] Fuks D, Regimbeau JM, Pessaux P et al. Is port-site resection necessary in the surgical management of gallbladder cancer? J Visc Surg 2013; 150: $277-284$

[631] D'Angelica M, Dalal KM, DeMatteo RP et al. Analysis of the extent of resection for adenocarcinoma of the gallbladder. Ann Surg Oncol 2009; 16: $806-816$

[632] Yi X, Long X, Zai $\mathrm{H}$ et al. Unsuspected gallbladder carcinoma discovered during or after cholecystectomy: focus on appropriate radical reresection according to the T-stage. Clin Transl Oncol 2013; 15: 652 658

[633] D’Hondt M, Lapointe R, Benamira Z et al. Carcinoma of the gallbladder: patterns of presentation, prognostic factors and survival rate. An 11-year single centre experience. Eur J Surg Oncol 2013; 39: 548 - 553

[634] Hari DM, Howard JH, Leung AM et al. A 21-year analysis of stage I gallbladder carcinoma: is cholecystectomy alone adequate? HPB (Oxford) 2013; 15: 40-48

[635] Andren-Sandberg A, Deng Y. Aspects on gallbladder cancer in 2014. Curr Opin Gastroenterol 2014; 30: 326-331

[636] Jayaraman S, Jarnagin WR. Management of gallbladder cancer. Gastroenterol Clin North Am 2010; 39: 331 - 342

[637] Götze TO, Paolucci V. Influence of high- and low-volume liver surgery in gallbladder carcinoma. World J Gastroenterol 2014; 20: $18445-$ 18451

[638] Götze TO, Paolucci V. Adequate extent in radical re-resection of incidental gallbladder carcinoma: analysis of the German Registry. Surg Endosc 2010; 24: 2156-2164

[639] Ethun CG, Postlewait LM, Le N et al. Association of optimal time interval to re-resection for incidental gallbladder cancer with overall survival: a multi-institution analysis from the US extrahepatic biliary malignancy consortium. JAMA Surg 2017; 152: 143 - 149

[640] Kohya N, Miyazaki K. Hepatectomy of segment 4a and 5 combined with extra-hepatic bile duct resection for T2 and T3 gallbladder carcinoma. J Surg Oncol 2008; 97: 498-502

[641] Araida T, Higuchi R, Hamano M et al. Hepatic resection in 485 R0 pT2 and PT3 cases of advanced carcinoma of the gallbladder: results of a Japanese Society of Biliary Surgery survey-a multicenter study. J Hepatobiliary Pancreat Surg 2009; 16: $204-215$

[642] Horiguchi A, Miyakawa S, Ishihara S et al. Gallbladder bed resection or hepatectomy of segments 4 a and 5 for PT2 gallbladder carcinoma: analysis of Japanese registration cases by the study group for biliary surgery of the Japanese Society of Hepato-Biliary-Pancreatic Surgery. J Hepatobiliary Pancreat Sci 2013; 20: 518- 524
[643] Pawlik TM, Gleisner AL, Vigano L et al. Incidence of finding residual disease for incidental gallbladder carcinoma: implications for re-resection. J Gastrointest Surg 2007; 11: 1478-1486, discussion 14861477

[644] Ko CW. Risk factors for gallstone-related hospitalization during pregnancy and the postpartum. Am J Gastroenterol 2006; 101: 2263 2268

[645] Swisher SG, Schmit PJ, Hunt KK et al. Biliary disease during pregnancy. Am J Surg 1994; 168: 576-579, discussion 580-571

[646] Lu EJ, Curet MJ, El-Sayed YY et al. Medical versus surgical management of biliary tract disease in pregnancy. Am J Surg 2004; 188: 755-759

[647] Jelin EB, Smink DS, Vernon AH et al. Management of biliary tract disease during pregnancy: a decision analysis. Surg Endosc 2008; 22: 54 60

[648] Dhupar R, Smaldone GM, Hamad GG. Is there a benefit to delaying cholecystectomy for symptomatic gallbladder disease during pregnancy? Surg Endosc 2010; 24: $108-112$

[649] Othman MO, Stone E, Hashimi M et al. Conservative management of cholelithiasis and its complications in pregnancy is associated with recurrent symptoms and more emergency department visits. Gastrointest Endosc 2012; 76: 564 - 569

[650] Jorge AM, Keswani RN, Veerappan A et al. Non-operative management of symptomatic cholelithiasis in pregnancy is associated with frequent hospitalizations. J Gastrointest Surg 2015; 19: 598-603

[651] Knab LM, Boller AM, Mahvi DM. Cholecystitis. Surg Clin North Am 2014; $94: 455-470$

[652] Athwal R, Bhogal RH, Hodson J et al. Surgery for gallstone disease during pregnancy does not increase fetal or maternal mortality: a metaanalysis. Hepatobiliary Surg Nutr 2016; 5: 53-57

[653] Cox TC, Huntington CR, Blair LJ et al. Laparoscopic appendectomy and cholecystectomy versus open: a study in 1999 pregnant patients. Surg Endosc 2016; 30: 593-602

[654] Pearl JP, Price RR, Tonkin AE et al. SAGES guidelines for the use of laparoscopy during pregnancy. Surg Endosc 2017; 31: 3767 - 3782

[655] Date RS, Kaushal M, Ramesh A. A review of the management of gallstone disease and its complications in pregnancy. Am J Surg 2008; 196: $599-608$

[656] Pearl J, Price R, Richardson W et al. Guidelines for diagnosis, treatment, and use of laparoscopy for surgical problems during pregnancy. Surg Endosc 2011; 25: 3479-3492

[657] Veerappan A, Gawron AJ, Soper NJ et al. Delaying cholecystectomy for complicated gallstone disease in pregnancy is associated with recurrent postpartum symptoms. J Gastrointest Surg 2013; 17: 1953 - 1959

[658] Bittner R. The standard of laparoscopic cholecystectomy. Langenbecks Arch Surg 2004; 389: 157 - 163

[659] Carus T. Current advances in single-port laparoscopic surgery. Langenbecks Arch Surg 2013; 398: 925 - 929

[660] van den Boezem PB, Velthuis S, Lourens HJ et al. Single-incision and NOTES cholecystectomy, are there clinical or cosmetic advantages when compared to conventional laparoscopic cholecystectomy? A case-control study comparing single-incision, transvaginal, and conventional laparoscopic technique for cholecystectomy. World J Surg 2014; 38: $25-32$

[661] Borchert DH, Federlein M, Fritze-Buttner F et al. Postoperative pain after transvaginal cholecystectomy: single-center, double-blind, randomized controlled trial. Surg Endosc 2014; 28: 1886-1894

[662] Milas M, Devedija S, Trkulja V. Single incision versus standard multiport laparoscopic cholecystectomy: up-dated systematic review and metaanalysis of randomized trials. Surgeon 2014; 12: $271-289$

[663] Lehmann KS, Zornig C, Arlt G et al. „Natural orifice transluminal endoscopic surgery“ in Deutschland. Chirurg 2015; 86: 577-586 
[664] Wood SG, Panait L, Duffy A] et al. Complications of transvaginal natural orifice transluminal endoscopic surgery: a series of 102 patients. Ann Surg 2014; 259: 744 - 749

[665] Markar SR, Karthikesalingam A, Thrumurthy S et al. Single-incision laparoscopic surgery (SILS) vs. conventional multiport cholecystectomy: systematic review and metaanalysis. Surg Endosc 2012; 26: $1205-$ 1213

[666] Luna RA, Nogueira DB, Varela PS et al. A prospective, randomized comparison of pain, inflammatory response, and short-term outcomes between single port and laparoscopic cholecystectomy. Surg Endosc 2013; 27: 1254-1259

[667] Haueter R, Schutz T, Raptis DA et al. Metaanalysis of single-port versus conventional laparoscopic cholecystectomy comparing body image and cosmesis. Br J Surg 2017; 104: 1141 - 1159

[668] Gurusamy KS, Vaughan J, Toon CD et al. Pharmacological interventions for prevention or treatment of postoperative pain in people undergoing laparoscopic cholecystectomy. Cochrane Database Syst Rev 2014; 3: CD008261

[669] Gurusamy KS, Nagendran M, Guerrini GP et al. Intraperitoneal local anaesthetic instillation versus no intraperitoneal local anaesthetic instillation for laparoscopic cholecystectomy. Cochrane Database Syst Rev 2014; 3: CD007337

[670] Loizides S, Gurusamy KS, Nagendran M et al. Wound infiltration with local anaesthetic agents for laparoscopic cholecystectomy. Cochrane Database Syst Rev 2014; 3: CD007049

[671] Lee YS, Kang BK, Hwang IK et al. Long-term outcomes of symptomatic gallbladder sludge. J Clin Gastroenterol 2015; 49: 594-598

[672] Raty S, Pulkkinen J, Nordback I et al. Can laparoscopic cholecystectomy prevent recurrent idiopathic acute pancreatitis?: a prospective randomized multicenter trial. Ann Surg 2015; 262: 736 - 741

[673] Shaffer EA. Gallbladder sludge: what is its clinical significance? Curr Gastroenterol Rep 2001; 3: 166-173

[674] Jüngst C, Kullak-Ublick GA, Jüngst D. Gallstone disease: microlithiasis and sludge. Best Pract Res Clin Gastroenterol 2006; 20: 1053-1062

[675] Eikermann M, Siegel R, Broeders I et al. Prevention and treatment of bile duct injuries during laparoscopic cholecystectomy: the clinical practice guidelines of the european association for endoscopic surgery (EAES). Surg Endosc 2012; 26: 3003-3039

[676] Gebhardt C, Meinl P. Lesions of the bile ducts in open cholecystectomy. Chirurg 1994; 65: 741 - 747

[677] Shea JA, Healey MJ, Berlin JA et al. Mortality and complications associated with laparoscopic cholecystectomy. A metaanalysis. Ann Surg 1996; 224: 609-620

[678] Wolnerhanssen BK, Ackermann C, Guenin MO et al. Zwölf Jahre laparoskopische Cholezystektomie. Chirurg 2005; 76: 263 -269

[679] Buddingh KT, Nieuwenhuijs VB, van Buuren L et al. Intraoperative assessment of biliary anatomy for prevention of bile duct injury: a review of current and future patient safety interventions. Surg Endosc 2011; 25: $2449-2461$

[680] Bockler D, Geoghegan J, Klein M et al. Implications of laparoscopic cholecystectomy for surgical residency training. JSLS 1999; 3: 19-22

[681] Borzellino G, de Manzoni G, Ricci F et al. Emergency cholecystostomy and subsequent cholecystectomy for acute gallstone cholecystitis in the elderly. Br J Surg 1999; 86: 1521 - 1525

[682] Wegrzyn P, Popiolek M, Przybylowski P et al. The risk of cholelithiasis in patients after heart transplantation. Arch Med Sci 2014; 10: 53-57

[683] Schiemann U, Ferhat A, Gotzberger M et al. Prevalence of cholecystolithiasis and its management among kidney/pancreas-transplanted type 1 (insulin-dependent) diabetic patients. Eur J Med Res 2008; 13: $127-130$

[684] Kilic A, Sheer A, Shah AS et al. Outcomes of cholecystectomy in US heart transplant recipients. Ann Surg 2013; 258: 312-317
[685] Begos DG, Franco KL, Baldwin JC et al. Optimal timing and indications for cholecystectomy in cardiac transplant patients. World J Surg 1995; 19: $661-667$

[686] Takeyama H, Sinanan MN, Fishbein DP et al. Expectant management is safe for cholelithiasis after heart transplant. J Heart Lung Transplant 2006; 25: 539-543

[687] Sarkio S, Salmela K, Kyllonen L et al. Complications of gallstone disease in kidney transplantation patients. Nephrol Dial Transplant 2007; 22: $886-890$

[688] Brito AT, Azevedo LS, Nahas WC et al. Cholelithiasis in patients on the kidney transplant waiting list. Clinics (Sao Paulo) 2010; 65: 389-391

[689] Leonardi M], Jamil KG, Hiscox B et al. Abdominal surgery after lung transplantation. Am Surg 2010; 76: 1130-1134

[690] Moller M, Gustafsson U, Rasmussen F et al. Natural course vs interventions to clear common bile duct stones: data from the Swedish registry for gallstone surgery and endoscopic retrograde cholangiopancreatografy (GallRiks). JAMA Surg 2014; 149: 1008-1013

[691] Saharia PC, Zuidema GD, Cameron JL. Primary common duct stones. Ann Surg 1977; 185: 598-604

[692] Swahn F, Nilsson M, Arnelo U et al. Rendezvous cannulation technique reduces post-ERCP pancreatitis: a prospective nationwide study of 12718 ERCP procedures. Am J Gastroenterol 2013; 108: 552 - 559

[693] Alexakis N, Connor S. Metaanalysis of one- vs. two-stage laparoscopic) endoscopic management of common bile duct stones. HPB (Oxford) 2012; 14: $254-259$

[694] Dasari BV, Tan C], Gurusamy KS et al. Surgical versus endoscopic treatment of bile duct stones. Cochrane Database Syst Rev 2013; 12: CD003327

[695] Baron TH, Harewood GC. Endoscopic balloon dilation of the biliary sphincter compared to endoscopic biliary sphincterotomy for removal of common bile duct stones during ERCP: a metaanalysis of randomized, controlled trials. Am J Gastroenterol 2004; 99: 1455 - 1460

[696] Zhao HC, He L, Zhou DC et al. Metaanalysis comparison of endoscopic papillary balloon dilatation and endoscopic sphincteropapillotomy. World J Gastroenterol 2013; 19: 3883-3891

[697] Doi S, Yasuda I, Mukai T et al. Comparison of long-term outcomes after endoscopic sphincterotomy versus endoscopic papillary balloon dilation: a propensity score-based cohort analysis. J Gastroenterol 2013; 48: 1090 - 1096

[698] Kim JH, Yang M], Hwang JC et al. Endoscopic papillary large balloon dilation for the removal of bile duct stones. World J Gastroenterol 2013; 19: $8580-8594$

[699] Kim TH, Kim JH, Seo DW et al. International consensus guidelines for endoscopic papillary large-balloon dilation. Gastrointest Endosc 2016; 83: $37-47$

[700] Liao WC, Tu YK, Wu MS et al. Balloon dilation with adequate duration is safer than sphincterotomy for extracting bile duct stones: a systematic review and metaanalyses. Clin Gastroenterol Hepatol 2012; 10: 1101 1109

[701] Yang XM, Hu B. Endoscopic sphincterotomy plus large-balloon dilation vs endoscopic sphincterotomy for choledocholithiasis: a metaanalysis. World J Gastroenterol 2013; 19: 9453-9460

[702] Jin PP, Cheng JF, Liu D et al. Endoscopic papillary large balloon dilation vs endoscopic sphincterotomy for retrieval of common bile duct stones: a metaanalysis. World J Gastroenterol 2014; 20: 5548-5556

[703] Stefanidis G, Viazis N, Pleskow D et al. Large balloon dilation vs. mechanical lithotripsy for the management of large bile duct stones: a prospective randomized study. Am J Gastroenterol 2011; 106: 278 285

[704] Ozawa N, Yasuda I, Doi S et al. Prospective randomized study of endoscopic biliary stone extraction using either a basket or a balloon catheter: the BasketBall study. J Gastroenterol 2017; 52: 623-630 
[705] Ishiwatari $\mathrm{H}$, Kawakami H, Hisai $\mathrm{H}$ et al. Balloon catheter versus basket catheter for endoscopic bile duct stone extraction: a multicenter randomized trial. Endoscopy 2016; 48: 350 - 357

[706] Jakobs R, Adamek HE, Maier M et al. Fluoroscopically guided laser lithotripsy versus extracorporeal shock wave lithotripsy for retained bile duct stones: a prospective randomised study. Gut 1997; 40: 678-682

[707] Neuhaus H, Zillinger C, Born P et al. Randomized study of intracorporeal laser lithotripsy versus extracorporeal shock-wave lithotripsy for difficult bile duct stones. Gastrointest Endosc 1998; 47: 327 - 334

[708] Sauerbruch T, Stern M. Fragmentation of bile duct stones by extracorporeal shock waves. A new approach to biliary calculi after failure of routine endoscopic measures. Gastroenterology 1989; 96: 146-152

[709] Sauerbruch T, Holl J, Sackmann M et al. Fragmentation of bile duct stones by extracorporeal shock-wave lithotripsy: a five-year experience. Hepatology 1992; 15: 208-214

[710] Adamek HE, Buttmann A, Wessbecher R et al. Clinical comparison of extracorporeal piezoelectric lithotripsy (EPL) and intracorporeal electrohydraulic lithotripsy $(\mathrm{EHL})$ in difficult bile duct stones. A prospective randomized trial. Dig Dis Sci 1995; 40: 1185-1192

[711] Adamek HE, Maier M, Jakobs R et al. Management of retained bile duct stones: a prospective open trial comparing extracorporeal and intracorporeal lithotripsy. Gastrointest Endosc 1996; 44: 40-47

[712] Ellis RD, Jenkins AP, Thompson RP et al. Clearance of refractory bile duct stones with extracorporeal shockwave lithotripsy. Gut 2000; 47: $728-731$

[713] Sackmann M, Holl J, Sauter GH et al. Extracorporeal shock wave lithotripsy for clearance of bile duct stones resistant to endoscopic extraction. Gastrointest Endosc 2001; 53: 27 - 32

[714] Adamek HE, Kudis V, Jakobs R et al. Impact of gallbladder status on the outcome in patients with retained bile duct stones treated with extracorporeal shockwave lithotripsy. Endoscopy 2002; 34: 624-627

[715] Clayton ES, Connor S, Alexakis N et al. Metaanalysis of endoscopy and surgery versus surgery alone for common bile duct stones with the gallbladder in situ. Br J Surg 2006; 93: 1185-1191

[716] Van der Velden J], Berger MY, Bonjer HJ et al. Percutaneous treatment of bile duct stones in patients treated unsuccessfully with endoscopic retrograde procedures. Gastrointest Endosc 2000; 51: 418 -422

[717] Garcia-Vila JH, Redondo-Ibanez M, Diaz-Ramon C. Balloon sphincteroplasty and transpapillary elimination of bile duct stones: 10 years' experience. Am J Roentgenol 2004; 182: 1451 - 1458

[718] Bergman J], Rauws EA, Tijssen JG et al. Biliary endoprostheses in elderly patients with endoscopically irretrievable common bile duct stones: report on 117 patients. Gastrointest Endosc 1995; 42: 195-201

[719] Maxton DG, Tweedle DE, Martin DF. Retained common bile duct stones after endoscopic sphincterotomy: temporary and longterm treatment with biliary stenting. Gut 1995; 36: 446-449

[720] Johnson GK, Geenen JE, Venu RP et al. Treatment of non-extractable common bile duct stones with combination ursodeoxycholic acid plus endoprostheses. Gastrointest Endosc 1993; 39: 528-531

[721] Pisello F, Geraci G, Li Volsi F et al. Permanent stenting in "unextractable" common bile duct stones in high risk patients. A prospective randomized study comparing two different stents. Langenbecks Arch Surg 2008; 393: $857-863$

[722] Boerma D, Rauws EA, Keulemans YC et al. Wait-and-see policy or laparoscopic cholecystectomy after endoscopic sphincterotomy for bileduct stones: a randomised trial. Lancet 2002; 360: 761 - 765

[723] Lau JY, Leow CK, Fung TM et al. Cholecystectomy or gallbladder in situ after endoscopic sphincterotomy and bile duct stone removal in Chinese patients. Gastroenterology 2006; 130: 96-103

[724] Heo J, Jung MK, Cho CM. Should prophylactic cholecystectomy be performed in patients with concomitant gallstones after endoscopic sphincterotomy for bile duct stones? Surg Endosc 2015; 29: 1574 1579

[725] Zargar SA, Mushtaq M, Beg MA et al. Wait-and-see policy versus cholecystectomy after endoscopic sphincterotomy for bile-duct stones in high-risk patients with co-existing gallbladder stones: a prospective randomised trial. Arab J Gastroenterol 2014; 15: 24-26

[726] Schiphorst AH, Besselink MG, Boerma D et al. Timing of cholecystectomy after endoscopic sphincterotomy for common bile duct stones. Surg Endosc 2008; 22: 2046-2050

[727] Huang RJ, Barakat MT, Girotra M et al. Practice patterns for cholecystectomy after endoscopic retrograde cholangiopancreatografy for patients with choledocholithiasis. Gastroenterology 2017; 153: 762 771.e762

[728] Reinders JS, Goud A, Timmer R et al. Early laparoscopic cholecystectomy improves outcomes after endoscopic sphincterotomy for choledochocystolithiasis. Gastroenterology 2010; 138: 2315-2320

[729] Fölsch UR, Nitsche R, Lüdtke R et al. Early ERCP and papillotomy compared with conservative treatment for acute biliary pancreatitis. The German study group on acute biliary pancreatitis. N Engl J Med 1997; 336: $237-242$

[730] Petrov MS, van Santvoort HC, Besselink MG et al. Early endoscopic retrograde cholangiopancreatografy versus conservative management in acute biliary pancreatitis without cholangitis: a metaanalysis of randomized trials. Ann Surg 2008; 247: 250-257

[731] Aboulian A, Chan T, Yaghoubian A et al. Early cholecystectomy safely decreases hospital stay in patients with mild gallstone pancreatitis: a randomized prospective study. Ann Surg 2010; 251: 615-619

[732] da Costa DW, Dijksman LM, Bouwense SA et al. Cost-effectiveness of same-admission versus interval cholecystectomy after mild gallstone pancreatitis in the PONCHO trial. Br J Surg 2016; 103: 1695- 1703

[733] Bradley EL. A clinically based classification system for acute pancreatitis. Summary of the International Symposium on Acute Pancreatitis, Atlanta, GA, September 11 through 13, 1992. Arch Surg 1993; 128: $586-590$

[734] Whitcomb DC. Clinical practice. Acute pancreatitis. N Engl] Med 2006; 354: $2142-2150$

[735] Tenner S, Baillie J, DeWitt J et al. American College of Gastroenterology guideline: management of acute pancreatitis. Am J Gastroenterol 2013; 108: $1400-1415 ; 1416$

[736] Working Group IAP/APA. Acute pancreatitis guidelines. IAP/APA evidence-based guidelines for the management of acute pancreatitis. Pancreatology 2013; 13: e1 - e15

[737] Ebramzadeh E, Campbell P, Tan TL et al. Can wear explain the histological variation around metal-on-metal total hips? Clin Orthop Relat Res 2015; 473: 487 - 494

[738] Wilson CT, de Moya MA. Cholecystectomy for acute gallstone pancreatitis: early vs delayed approach. Scand J Surg 2010; 99: 81 - 85

[739] Neoptolemos JP, Carr-Locke DL, London NJ et al. Controlled trial of urgent endoscopic retrograde cholangiopancreatografy and endoscopic sphincterotomy versus conservative treatment for acute pancreatitis due to gallstones. Lancet 1988; 2: 979-983

[740] Fan ST, Lai EC, Mok FP et al. Early treatment of acute biliary pancreatitis by endoscopic papillotomy. N Engl J Med 1993; 328: 228-232

[741] Ayub K, Imada R, Slavin J. Endoscopic retrograde cholangiopancreatografy in gallstone-associated acute pancreatitis. Cochrane Database Syst Rev 2004; 4: CD003630

[742] Oria A, Cimmino D, Ocampo C et al. Early endoscopic intervention versus early conservative management in patients with acute gallstone pancreatitis and biliopancreatic obstruction: a randomized clinical trial. Ann Surg 2007; 245: $10-17$

[743] Lai EC, Mok FP, Tan ES et al. Endoscopic biliary drainage for severe acute cholangitis. N Engl J Med 1992; 326: $1582-1586$ 
[744] Acosta JM, Katkhouda N, Debian KA et al. Early ductal decompression versus conservative management for gallstone pancreatitis with ampullary obstruction: a prospective randomized clinical trial. Ann Surg 2006; 243: $33-40$

[745] Sawas T, Arwani N, Al Halabi S et al. Sphincterotomy with endoscopic biliary drainage for severe acute cholangitis: a metaanalysis. Endosc Int Open 2017; 5: E103-E109

[746] Fusaroli P, Jenssen C, Hocke $M$ et al. EFSUMB guidelines on interventional ultrasound (INVUS), part V. Ultraschall in Med 2016; 37: 77 - 99

[747] Sharaiha RZ, Khan MA, Kamal F et al. Efficacy and safety of EUS-guided biliary drainage in comparison with percutaneous biliary drainage when ERCP fails: a systematic review and metaanalysis. Gastrointest Endosc 2017; 85: $904-914$

[748] Khan MA, Akbar A, Baron TH et al. Endoscopic ultrasound-guided biliary drainage: a systematic review and metaanalysis. Dig Dis Sci 2016; 61: $684-703$

[749] Mayumi T, Okamoto K, Takada T et al. Tokyo guidelines 2018 management bundles for acute cholangitis and cholecystitis. J Hepatobiliary Pancreat Sci 2018; 25: 96 - 100

[750] Harris A, Chan AC, Torres-Viera C et al. Metaanalysis of antibiotic prophylaxis in endoscopic retrograde cholangiopancreatografy (ERCP). Endoscopy 1999; 31: 718-724

[751] Vaughan J, Gurusamy KS, Davidson BR. Day-surgery versus overnight stay surgery for laparoscopic cholecystectomy. Cochrane Database Syst Rev 2013; 7: CD006798

[752] Brand M, Bizos D, O’Farrell P et al. Antibiotic prophylaxis for patients undergoing elective endoscopic retrograde cholangiopancreatografy. Cochrane Database Syst Rev 2010; 10: CD007345

[753] Weickert U, Jakobs R, Hahne M et al. Cholangioskopie nach erfolgreicher Therapie einer komplizierten Choledocholithiasis. Dtsch Med Wochenschr 2003; 128: 481 -484

[754] Adler DG, Baron TH, Davila RE et al. ASGE guideline: the role of ERCP in diseases of the biliary tract and the pancreas. Gastrointest Endosc 2005; 62: $1-8$

[755] Efthymiou M, Raftopoulos S, Antonio Chirinos J et al. Air embolism complicated by left hemiparesis after direct cholangioscopy with an intraductal balloon anchoring system. Gastrointest Endosc 2012; 75: $221-223$

[756] Albert JG, Friedrich-Rust M, Elhendawy M et al. Peroral cholangioscopy for diagnosis and therapy of biliary tract disease using an ultra-slim gastroscope. Endoscopy 2011; 43: 1004-1009

[757] Winbladh A, Gullstrand P, Svanvik J et al. Systematic review of cholecystostomy as a treatment option in acute cholecystitis. HPB (Oxford) 2009; 11: 183-193

[758] McGillicuddy EA, Schuster KM, Barre K et al. Non-operative management of acute cholecystitis in the elderly. Br J Surg 2012; 99: 1254 1261

[759] Akyürek N, Salman B, Yuksel O et al. Management of acute calculous cholecystitis in high-risk patients: percutaneous cholecystotomy followed by early laparoscopic cholecystectomy. Surg Laparosc Endosc Percutan Tech 2005; 15: 315-320

[760] Hatzidakis AA, Prassopoulos P, Petinarakis I et al. Acute cholecystitis in high-risk patients: percutaneous cholecystostomy vs conservative treatment. Eur Radiol 2002; 12: $1778-1784$

[761] Gurusamy KS, Rossi M, Davidson BR. Percutaneous cholecystostomy for high-risk surgical patients with acute calculous cholecystitis. Cochrane Database Syst Rev 2013; 8: CD007088

[762] Ambe PC, Kaptanis S, Papadakis M et al. The Treatment of Critically III Patients With Acute Cholecystitis. Dtsch Arztebl Int 2016; 113: 545 551
[763] Jang JW, Lee SS, Song T] et al. Endoscopic ultrasound-guided transmural and percutaneous transhepatic gallbladder drainage are comparable for acute cholecystitis. Gastroenterology 2012; 142: 805-811

[764] Itoi T, Kawakami H, Katanuma A et al. Endoscopic nasogallbladder tube or stent placement in acute cholecystitis: a preliminary prospective randomized trial in Japan (with videos). Gastrointest Endosc 2015; 81: $111-118$

[765] Yang MJ, Yoo BM, Kim JH et al. Endoscopic naso-gallbladder drainage versus gallbladder stenting before cholecystectomy in patients with acute cholecystitis and a high suspicion of choledocholithiasis: a prospective randomised preliminary study. Scand ] Gastroenterol 2016; 51: $472-478$

[766] Mori Y, Itoi T, Baron TH et al. TG18 management strategies for gallbladder drainage in patients with acute cholecystitis: updated Tokyo guidelines 2018 (with videos). J Hepatobiliary Pancreat Sci 2018; 25: $87-95$

[767] Anderloni A, Buda A, Vieceli F et al. Endoscopic ultrasound-guided transmural stenting for gallbladder drainage in high-risk patients with acute cholecystitis: a systematic review and pooled analysis. Surg Endosc 2016; 30: 5200-5208

[768] Teoh AYB, Serna C, Penas I et al. Endoscopic ultrasound-guided gallbladder drainage reduces adverse events compared with percutaneous cholecystostomy in patients who are unfit for cholecystectomy. Endoscopy 2017; 49: 130-138

[769] Irani S, Ngamruengphong S, Teoh A et al. Similar efficacies of endoscopic ultrasound gallbladder drainage with a lumen-apposing metal stent versus percutaneous transhepatic gallbladder drainage for acute cholecystitis. Clin Gastroenterol Hepatol 2017; 15: 738- 745

[770] Dollhopf M, Larghi A, Will U et al. EUS-guided gallbladder drainage in patients with acute cholecystitis and high surgical risk using an electrocautery-enhanced lumen-apposing metal stent device. Gastrointest Endosc 2017; 86: 636-643

[771] Tyberg A, Saumoy M, Sequeiros EV et al. EUS-guided versus percutaneous gallbladder drainage: isn't it time to convert? J Clin Gastroenterol 2018; 52: 79-84

[772] Khan MA, Atiq O, Kubiliun N et al. Efficacy and safety of endoscopic gallbladder drainage in acute cholecystitis: Is it better than percutaneous gallbladder drainage? Gastrointest Endosc 2017; 85: 76-87. e73

[773] Kahaleh M, Perez-Miranda M, Artifon EL et al. International collaborative study on EUS-guided gallbladder drainage: Are we ready for prime time? Dig Liver Dis 2016; 48: 1054-1057

[774] Elshaer M, Gravante G, Thomas K et al. Subtotal cholecystectomy for "difficult gallbladders": systematic review and metaanalysis. JAMA Surg 2015; 150: 159-168

[775] Kim KH, Sung CK, Park BG et al. Clinical significance of intrahepatic biliary stricture in efficacy of hepatic resection for intrahepatic stones. J Hepatobiliary Pancreat Surg 1998; 5: 303 - 308

[776] Cheon YK, Cho YD, Moon JH et al. Evaluation of long-term results and recurrent factors after operative and nonoperative treatment for hepatolithiasis. Surgery 2009; 146: $843-853$

[777] Axelrad AM, Fleischer DE, Strack LL et al. Performance of ERCP for symptomatic choledocholithiasis during pregnancy: techniques to increase safety and improve patient management. Am J Gastroenterol 1994; 89: 109-112

[778] Jamidar PA, Beck G], Hoffman B] et al. Endoscopic retrograde cholangiopancreatografy in pregnancy. Am J Gastroenterol 1995; 90: 1263 1267

[779] Sungler P, Heinerman PM, Steiner H et al. Laparoscopic cholecystectomy and interventional endoscopy for gallstone complications during pregnancy. Surg Endosc 2000; 14: 267 - 271

[780] Tham TC, Vandervoort ], Wong RC et al. Safety of ERCP during pregnancy. Am J Gastroenterol 2003; 98: 308-311 
[781] Kahaleh M, Hartwell GD, Arseneau KO et al. Safety and efficacy of ERCP in pregnancy. Gastrointest Endosc 2004; 60: 287-292

[782] Menees S, Elta G. Endoscopic retrograde cholangiopancreatografy during pregnancy. Gastrointest Endosc Clin N Am 2006; 16: 41 - 57

[783] Jackson H, Granger S, Price R et al. Diagnosis and laparoscopic treatment of surgical diseases during pregnancy: an evidence-based review. Surg Endosc 2008; 22: 1917 - 1927

[784] Tang SJ, Mayo MJ, Rodriguez-Frias E et al. Safety and utility of ERCP during pregnancy. Gastrointest Endosc 2009; 69: 453-461

[785] Chong VH, Jalihal A. Endoscopic management of biliary disorders during pregnancy. Hepatobiliary Pancreat Dis Int 2010; 9: 180-185

[786] Fine S, Beirne J, Delgi-Esposti S et al. Continued evidence for safety of endoscopic retrograde cholangiopancreatografy during pregnancy. World J Gastrointest Endosc 2014; 6: 352 - 358

[787] Inamdar S, Berzin TM, Sejpal DV et al. Pregnancy is a risk factor for pancreatitis after endoscopic retrograde cholangiopancreatografy in a national cohort study. Clin Gastroenterol Hepatol 2016; 14: 107-114

[788] Freistühler M, Braess A, Petrides AS. Ultraschallgeleitete endoskopische Papillotomie in der Schwangerschaft bei schwerer biliärer Pankreatitis. Z Gastroenterol 1999; 37: 27-30

[789] Röntgengesellschaft D, Physik DGfM. Pränatale Strahlenexposition aus medizinischer Indikation. Dosisermittlung, Folgerungen für Arzt und Schwangere. Berlin: DGMP. 2002

[790] AQUA-Institut für angewandte Qualitätsförderung und Forschung im Gesundheitswesen. Sektorenübergreifende Qualitätssicherung im Gesundheitswesen nach §137a SGB V. Bericht zur Prüfung und Bewertung der Indikatoren der externen stationären Qualitätssicherung hinsichtlich ihrer Eignung für die einrichtungsbezogene öffentliche Berichterstattung. Göttingen, 2015

[791] AQUA-Institut für angewandte Qualitätsförderung und Forschung im Gesundheitswesen. Sektorenübergreifende Qualitätssicherung im Gesundheitswesen nach §137a SGB V. Weiterentwicklung des Leistungsbereichs Cholezystektomie. Ergebnisbericht. Göttingen, 2013

[792] Al-Kubati WR. Bile duct injuries following laparoscopic cholecystectomy: a clinical study. Saudi J Gastroenterol 2010; 16: 100 -104

[793] AQUA-Institut für angewandte Qualitätsförderung und Forschung im Gesundheitswesen. Bundesauswertung zum Erfassungsjahr 2014. 12/ 1 - Cholezystektomie. Qualitätsindikatoren. Göttingen, 2015

[794] Tantia O, Jain M, Khanna S et al. latrogenic biliary injury: 13305 cholecystectomies experienced by a single surgical team over more than 13 years. Surg Endosc 2008; 22: 1077 - 1086

[795] Spelsberg FW, Nusser F, Huttl TK et al. Aktuelle Therapie der Cholezysto- und Choledocholithiasis - Umfrageergebnisse mit Analyse von 16615 Eingriffen in Bayern. Zentralbl Chir 2009; 134: 120-126

[796] Boerma D, Rauws EA, Keulemans YC et al. Impaired quality of life 5 years after bile duct injury during laparoscopic cholecystectomy: a prospective analysis. Ann Surg 2001; 234: 750 - 757

[797] Antoniou SA, Pointner R, Granderath FA. Single-incision laparoscopic cholecystectomy: a systematic review. Surg Endosc 2011; 25: 367 377

[798] Saeb-Parsy K, Mills A, Rang C et al. Emergency laparoscopic cholecystectomy in an unselected cohort: a safe and viable option in a specialist centre. Int J Surg 2010; 8: 489-493

[799] Ros A, Haglund B, Nilsson E. Reintervention after laparoscopic and open cholecystectomy in Sweden 1987-1995: analysis of data from a hospital discharge register. Eur J Surg 2002; 168: 695-700

[800] Heller G, Jeschke E. Einrichtungsübergreifende Qualitätssicherung der Gallenblasenentfernung auf der Basis von Routinedaten. Stuttgart: Schattauer. 2012

[801] Nimptsch U, Mansk T. Deaths Following Cholecystectomy and Herniotomy: An Analysis of Nationwide German Hospital Discharge Data From 2009 to 2013. Dtsch Arztebl Int 2015; 112: 535-543
[802] Nimptsch U, Mansky T. Hospital volume and mortality for 25 types of inpatient treatment in German hospitals: observational study using complete national data from 2009 to 2014. BMJ Open 2017; 7: e016184

[803] Ainslie WG, Catton JA, Davides D et al. Micropuncture cholecystectomy vs conventional laparoscopic cholecystectomy: a randomized controlled trial. Surg Endosc 2003; 17: $766-772$

[804] Nilsson E, Ros A, Rahmqvist M et al. Cholecystectomy: costs and health-related quality of life: a comparison of two techniques. Int J Qual Health Care 2004; 16: 473-482

[805] Abd Ellatif ME, Askar WA, Abbas AE et al. Quality-of-life measures after single-access versus conventional laparoscopic cholecystectomy: a prospective randomized study. Surg Endosc 2013; 27: 1896 - 1906

[806] Keus F, de Vries J, Gooszen HG et al. Laparoscopic versus small-incision cholecystectomy: health status in a blind randomised trial. Surg Endosc 2008; 22: 1649-1659

[807] Ma J, Cassera MA, Spaun GO et al. Randomized controlled trial comparing single-port laparoscopic cholecystectomy and four-port laparoscopic cholecystectomy. Ann Surg 2011; 254: 22 - 27

[808] Nicholl JP, Brazier JE, Milner PC et al. Randomised controlled trial of cost-effectiveness of lithotripsy and open cholecystectomy as treatments for gallbladder stones. Lancet 1992; 340: 801-807

[809] Vetrhus M, Soreide O, Eide GE et al. Pain and quality of life in patients with symptomatic, non-complicated gallbladder stones: results of a randomized controlled trial. Scand J Gastroenterol 2004; 39: 270 - 276

[810] Vetrhus M, Soreide O, Eide GE et al. Quality of life and pain in patients with acute cholecystitis. Results of a randomized clinical trial. Scand J Surg 2005; 94: $34-39$

[811] Barkun AN, Barkun JS, Sampalis JS et al. Costs and effectiveness of extracorporeal gallbladder stone shock wave lithotripsy versus laparoscopic cholecystectomy. A randomized clinical trial. McGill Gallstone Treatment Group. Int J Technol Assess Health Care 1997; 13: 589-601

[812] Brown KM, Moore BT, Sorensen GB et al. Patient-reported outcomes after single-incision versus traditional laparoscopic cholecystectomy: a randomized prospective trial. Surg Endosc 2013; 27: 3108-3115

[813] Russell ML, Preshaw RM, Brant RF et al. Disease-specific quality of life: the Gallstone Impact Checklist. Clin Invest Med 1996; 19: 453 - 460

[814] Chen TY, Landmann MG, Potter JC et al. Questionnaire to aid priority and outcomes assessment in gallstone disease. ANZ J Surg 2006; 76 : $569-574$

[815] NICE. Guide to the methods of technology appraisal. $2013 \mathrm{http}: / / \mathrm{pu}-$ blications.nice.org.uk/guide-to-the-methods-of-technology-appraisal-2013-pmg9/

[816] Longworth L, Yang Y, Young T et al. Use of generic and conditionspecific measures of health-related quality of life in NICE decisionmaking: a systematic review, statistical modelling and survey. Health Technol Assess 2014; 18: 1 - 224

[817] Shi HY, Lee HH, Chiu CC et al. Responsiveness and minimal clinically important differences after cholecystectomy: GIQLI versus SF-36. J Gastrointest Surg 2008; 12: 1275-1282

[818] Shi HY, Lee KT, Lee HH et al. The minimal clinically important difference in the Gastrointestinal Quality-of-Life Index after cholecystectomy. Surg Endosc 2009; 23: 2708-2712

[819] Smith KW, Avis NE, Assmann SF. Distinguishing between quality of life and health status in quality of life research: a metaanalysis. Qual Life Res 1999; 8: 447 - 459

[820] Covinsky KE, Wu AW, Landefeld CS et al. Health status versus quality of life in older patients: does the distinction matter? Am J Med 1999; 106 : $435-440$

[821] Kratzer W, Mason RA, Kachele V. Prevalence of gallstones in sonografic surveys worldwide. J Clin Ultrasound 1999; 27: 1-7 
[822] Paolucci V, Neckell M, Götze T. Das „okkulte“ Gallenblasenkarzinom CAE-S/CAMIC -Zentralregister. Zentralbl Chir 2003; 128: 309-312

[823] Wani S, Hall M, Keswani RN et al. Variation in aptitude of trainees in endoscopic ultrasonografy, based on cumulative sum analysis. Clin Gastroenterol Hepatol 2015; 13: 1318-1325.e1312

[824] Shahidi N, Ou G, Lam E et al. When trainees reach competency in performing endoscopic ultrasound: a systematic review. Endosc Int Open 2017; 5: E239-E243

[825] Noh KW, Woodward TA, Raimondo M et al. Changing trends in endosonografy: linear imaging and tissue are increasingly the issue. Dig Dis Sci 2007; 52: 1014-1018

[826] DeBenedet AT, Elmunzer BJ, McCarthy ST et al. Intraprocedural quality in endoscopic retrograde cholangiopancreatografy: a metaanalysis. Am J Gastroenterol 2013; 108: 1696-1704

[827] Adler DG, Lieb JG 2nd, Cohen J et al. Quality indicators for ERCP. Gastrointest Endosc 2015; 81: 54-66

[828] Dumonceau JM, Garcia-Fernandez F], Verdun FR et al. Radiation protection in digestive endoscopy: European society of digestive endoscopy (ESGE) guideline. Endoscopy 2012; 44: 408 -421

[829] Elfant AB, Bourke M], Alhalel $R$ et al. A prospective study of the safety of endoscopic therapy for choledocholithiasis in an outpatient population. Am J Gastroenterol 1996; 91: 1499-1502

[830] Freeman ML, Nelson DB, Sherman S et al. Same-day discharge after endoscopic biliary sphincterotomy: observations from a prospective multicenter complication study. The multicenter endoscopic sphincterotomy (MESH) study group. Gastrointest Endosc 1999; 49: $580-586$
[831] Johansson M, Thune A, Nelvin L et al. Randomized clinical trial of daycare versus overnight-stay laparoscopic cholecystectomy. $\mathrm{Br}$ J Surg 2006; 93: $40-45$

[832] Richardson WS, Fuhrman GS, Burch E et al. Outpatient laparoscopic cholecystectomy. Outcomes of 847 planned procedures. Surg Endosc 2001; 15: 193-195

[833] Rosen M], Malm JA, Tarnoff M et al. Cost-effectiveness of ambulatory laparoscopic cholecystectomy. Surg Laparosc Endosc Percutan Tech 2001; 11: $182-184$

[834] Maggiore D. Outpatient laparoscopic cholecystectomy: a reality. JSLS 2002; 6: 369-371

[835] Feussner H. Laparoskopische Eingriffe. Möglichkeiten und Grenzen der ambulanten und kurzstationären Chirurgie. Chirurg 2004; 75: 248 256

[836] Lillemoe KD, Lin JW, Talamini MA et al. Laparoscopic cholecystectomy as a "true" outpatient procedure: initial experience in 130 consecutive patients. J Gastrointest Surg 1999; 3: 44-49

[837] Blatt A, Chen S. Day-only laparoscopic cholecystectomy in a regional teaching hospital. ANZ J Surg 2003; 73: 321-325

[838] Banks PA, Bollen TL, Dervenis C et al. Classification of acute pancreatitis-2012: revision of the Atlanta classification and definitions by international consensus. Gut 2013; 62: $102-111$

[839] Marshall JC, Cook D], Christou NV et al. Multiple organ dysfunction score: a reliable descriptor of a complex clinical outcome. Crit Care Med 1995; 23: $1638-1652$ 